\title{
EMBEDDINGS OF FUNCTION SPACES VIA THE CAFFARELLI-SILVESTRE EXTENSION, CAPACITIES AND WOLFF POTENTIALS
}

\author{
PENGTAO LI, SHAOGUANG SHI, RUI HU, AND ZHICHUN ZHAI
}

\begin{abstract}
Let $P_{\alpha} f(x, t)$ be the Caffarelli-Silvestre extension of a smooth function $f(x): \mathbb{R}^{n} \rightarrow \mathbb{R}_{+}^{n+1}:=$ $\mathbb{R}^{n} \times(0, \infty)$. The purpose of this article is twofold. Firstly, we want to characterize a nonnegative measure $\mu$ on $\mathbb{R}_{+}^{n+1}$ such that $f(x) \rightarrow P_{\alpha} f(x, t)$ induces bounded embeddings from the Lebesgue spaces $L^{p}\left(\mathbb{R}^{n}\right)$ to the $L^{q}\left(\mathbb{R}_{+}^{n+1}, \mu\right)$. On one hand, these embeddings will be characterized by using a newly introduced $L^{p}$-capacity associated with the Caffarelli-Silvestre extension. In doing so, the mixed norm estimates of $P_{\alpha} f(x, t)$, the dual form of the $L^{p}$-capacity, the $L^{p}$-capacity of general balls, and a capacitary strong type inequality will be established, respectively. On the other hand, when $p>q>1$, these embeddings will also be characterized in terms of the Hedberg-Wolff potential of $\mu$. Secondly, we characterize a nonnegative measure $\mu$ on $\mathbb{R}_{+}^{n+1}$ such that $f(x) \rightarrow P_{\alpha} f(x, t)$ induces bounded embeddings from the homogeneous Sobolev spaces $\dot{W}^{\beta, p}\left(\mathbb{R}^{n}\right)$ to the $L^{q}\left(\mathbb{R}_{+}^{n+1}, \mu\right)$ in terms of the fractional perimeter of open sets for endpoint cases and the fractional capacity for general cases.
\end{abstract}

\section{CONTENTS}

1. Introduction

2. Preliminaries on capacities

2.1. $L^{p}$ - capacities associated with the Caffarelli-Silvestre extension

2.2. Capacitary strong type inequality

2.3. Preliminary lemmas on the fractional capacity

3. Embeddings of $L^{p}\left(\mathbb{R}^{n}\right)$ to $L^{q}\left(\mathbb{R}_{+}^{n+1}, \mu\right)$

3.1. Embeddings of $L^{p}\left(\mathbb{R}^{n}\right)$ to $L^{q}\left(\mathbb{R}_{+}^{n+1}, \mu\right)$ when $p \leq q$

3.2. Embeddings of $L^{p}\left(\mathbb{R}^{n}\right)$ to $L^{q}\left(\mathbb{R}_{+}^{n+1}, \mu\right)$ when $p>q$

4. Embeddings of $\dot{W}^{\beta, p}\left(\mathbb{R}^{n}\right)$ to $L^{q}\left(\mathbb{R}_{+}^{n+1}, \mu\right)$

4.1. Embeddings of $\dot{W}^{\beta, p}\left(\mathbb{R}^{n}\right)$ to $L^{q}\left(\mathbb{R}_{+}^{n+1}, \mu\right)$ when $1 \leq p \leq \min \{n / \beta, q\}$

4.2. Embeddings of $\dot{W}^{\beta, p}\left(\mathbb{R}^{n}\right)$ to $L^{q}\left(\mathbb{R}_{+}^{n+1}, \mu\right)$ when $\max \{q, 1\}<p \leq n / \beta$

Acknowledgement $\quad 39$

References

(P. Li) School of Mathematics and Statistics, Qingdao University, Qingdao, Shandong 266071, China

(S. Shi) Department of Mathematics, Linyi University, Linyi, 276005, China

(R. Hu) Department of Mathematics and Statistics, MacEwan University Edmonton, Alberta T5J2P2 Canada

(Z. Zhai) Department of Mathematics and Statistics, MacEwan University Edmonton, Alberta T5J2P2 Canada

E-mail addresses: ptli@qdu.edu.cn, shishaoguang@lyu.edu.cn, hur3@macewan.ca, zhaiz2@macewan.ca.

2010 Mathematics Subject Classification. Primary 31, 35J; Secondary: $42 B 37$.

Key words and phrases. Fractional Laplacian, Lebesgue space, Sobolev space, Capacity, Fractional perimeter.

Project supported: Pengtao Li was in part supported by the National Natural Science Foundation of China (\# 11871293 \& \# 11571217) \& Shandong Natural Science Foundation of China (\# ZR2017JL008, \# ZR2016AM05). 


\section{INTRODUCTION}

The fractional Laplace operator $(-\Delta)^{\frac{\alpha}{2}}$ for $\alpha \in(0,2)$ in $\mathbb{R}^{n}$ is defined on the Schwartz class through the Fourier transform as

$$
\left(\left(-\widehat{-\triangle)^{\frac{\alpha}{2}}} f\right)(\xi)=(2 \pi|\xi|)^{\alpha} \widehat{f}(\xi),\right.
$$

where $\widehat{f}(\xi)=\int_{\mathbb{R}^{n}} e^{-2 \pi i x \cdot \xi} f(x) d x$ is the Fourier transform of $f$, or via the Riesz potential as

$$
(-\Delta)^{\frac{\alpha}{2}} f(x)=\frac{\alpha 2^{\alpha} \Gamma((n+\alpha) / 2)}{2 \Gamma(1-\alpha / 2) \pi^{n / 2}} \text { P.V. } \int_{\mathbb{R}^{n}} \frac{f(x)-f(y)}{|x-y|^{n+\alpha}} d y .
$$

Here $\Gamma(\cdot)$ is the usual Gamma function and P.V. denotes the Cauchy principal value. The fractional Laplacian has been widely applied in probability, finance, physical systems, and engineering problems.

The fractional Laplacian is a nonlocal operator because the value of $(-\Delta)^{\frac{\alpha}{2}} f$ at $x$ depends on the value of $f$ at infinity. This nonlocal property may cause some issues. Caffarelli and Silvestre in [11] localized the nonlocal operator $(-\Delta)^{\frac{\alpha}{2}}$ by adding another variable. They provided the characterization for the fractional Laplacian $(-\triangle)^{\frac{\alpha}{2}}$ by solving the harmonic extension problem to the upper half-space as the weighted operator that maps the Dirichlet boundary condition to the Neumann condition.

Let $f$ be a regular function in $\mathbb{R}^{n}$. We say that $u(x, t)=P_{\alpha} f(x, t)$ is the Caffarelli-Silvestre extension of $f$ to the upper half-space $\mathbb{R}_{+}^{n+1}:=\mathbb{R}^{n} \times(0, \infty)$, if $u$ is a solution to the problem

$$
\left\{\begin{array}{rll}
\operatorname{div}\left(t^{1-\alpha} \nabla u\right)=0, & \text { in } \quad \mathbb{R}_{+}^{n+1} ; \\
u=f, & \text { on } \quad \mathbb{R}^{n} \times\{t=0\} .
\end{array}\right.
$$

The Caffarelli-Silvestre extension is well defined for smooth functions through the Poisson kernel

$$
p_{t}^{\alpha}(x)=\frac{c(n, \alpha) t^{\alpha}}{\left(|x|^{2}+t^{2}\right)^{(n+\alpha) / 2}}
$$

as

$$
P_{\alpha} f(x, t)=p_{t}^{\alpha} * f(x, t)=c(n, \alpha) \int_{\mathbb{R}^{n}} \frac{f(y) t^{\alpha}}{\left(|x-y|^{2}+t^{2}\right)^{(n+\alpha) / 2}} d y .
$$

Here $f * g$ means the convolution of $f$ and $g$, and $c(n, \alpha)=\frac{\Gamma((n+\alpha) / 2)}{\pi^{n / 2} \Gamma(\alpha / 2)}$ is the normalized constant such that $\int_{\mathbb{R}^{n}} p_{t}^{\alpha}(x) d x=1$. Caffarelli and Silvestre [11] proved that

$$
(-\triangle)^{\frac{\alpha}{2}} f(x)=-c_{\alpha} \lim _{t \rightarrow 0^{+}} t^{1-\alpha} \partial_{t} u(x, t), c_{\alpha}=\frac{\Gamma(\alpha / 2)}{2^{1-\alpha} \Gamma(1-\alpha / 2)} .
$$

This characterization has dramatically popularized the application of the fractional Laplacian.

The identity (1.2) can be viewed as the consequence of the equality of the energy functionals

$$
\int_{\mathbb{R}^{n}}|2 \pi \xi|^{\alpha}|\widehat{f}(\xi)|^{2} d \xi=D(n, \alpha) \int_{\mathbb{R}_{+}^{n+1}}\left|\nabla P_{\alpha} f(x, t)\right|^{2} t^{1-\alpha} d x d t
$$

which is equivalent to

$$
\|f\|_{\dot{W}^{\alpha / 2,2}\left(\mathbb{R}^{n}\right)}=\left\|P_{\alpha} f(x, t)\right\|_{\dot{W}_{\alpha}^{1,2}\left(\mathbb{R}_{+}^{n+1}\right)}
$$

up to a multiplication constant. Here, $\dot{\mathbb{W}}_{\alpha}^{1,2}\left(\mathbb{R}_{+}^{n+1}\right)$ is the weighted Sobolev space defined as

$$
\dot{\mathbb{W}}_{\alpha}^{1,2}\left(\mathbb{R}_{+}^{n+1}\right)=\left\{u(x, t) \in W_{l o c}^{1,1}\left(\mathbb{R}_{+}^{n+1}\right): \int_{\mathbb{R}_{+}^{n+1}}|\nabla u(x, t)|^{2} t^{1-\alpha} d x d t<\infty\right\} .
$$


Let $C_{0}^{\infty}\left(\mathbb{R}^{n}\right)$ stand for all infinitely smooth functions with compact support in $\mathbb{R}^{n}$. The homogeneous Sobolev space $\dot{W}^{\beta, p}\left(\mathbb{R}^{n}\right)$ is the completion of $C_{0}^{\infty}\left(\mathbb{R}^{n}\right)$ with respect to the norm

$$
\|f\|_{\dot{W}^{\beta, p}\left(\mathbb{R}^{n}\right)}=\left\{\begin{array}{l}
\left\|(-\Delta)^{\beta / 2} f\right\|_{L^{p}\left(\mathbb{R}^{n}\right)}, \quad p \in(1, n / \beta) ; \\
\left(\int_{\mathbb{R}^{n}} \frac{\left\|\Delta_{h}^{k} f\right\|_{L^{p}\left(\mathbb{R}^{n)}\right)}^{p}}{|h|^{n+p \beta}} d h\right)^{1 / p}, \quad p=1 \text { or } p=n / \beta, \beta \in(0, n),
\end{array}\right.
$$

where $k=1+[\beta], \beta=[\beta]+\{\beta\}$ with $[\beta] \in \mathbb{Z}_{+},\{\beta\} \in(0,1)$ and

$$
\triangle_{h}^{k} f(x)=\left\{\begin{array}{l}
\triangle_{h}^{1} \triangle_{h}^{k-1} f(x), \quad k>1 \\
f(x+h)-f(x), \quad k=1 .
\end{array}\right.
$$

Equality (1.3) allows us to identify fractional (logarithmic) Sobolev inequalities as fractional (logarithmic) Sobolev trace inequalities, see [8, 21]. It also provides us a way to view the fractional perimeters of a Borel set $E \subset \mathbb{R}^{n}$ as the norm of the Caffarelli-Silvestre extension of the $1_{E}$ (the characteristic function of $E$ ). See [10, 16] and the references therein for more details on the fractional perimeter.

Motivated by (1.3), in this paper, we characterize the following two embedding relations via the Caffarelli-Silvestre extension:

Embedding I: Given $\alpha \in(0,2)$ and a nonnegative Radon measure $\mu$ on $\mathbb{R}_{+}^{n+1}$,

$$
\left\|P_{\alpha} f(\cdot, \cdot)\right\|_{L^{q}\left(\mathbb{R}_{+}^{n+1}, \mu\right)} \lesssim\|f\|_{L^{p}\left(\mathbb{R}^{n}\right)} .
$$

For $0<p, q<\infty$ and a nonnegative Radon measure $\mu$ on $\mathbb{R}_{+}^{n+1}, L^{q, p}\left(\mathbb{R}_{+}^{n+1}, \mu\right)$ and $L^{q}\left(\mathbb{R}_{+}^{n+1}, \mu\right)$ denote the Lorentz space and the Lebesgue space of all functions on $\mathbb{R}_{+}^{n+1}$, respectively, for which

$$
\|g\|_{L^{q, p}\left(\mathbb{R}_{+}^{n+1}, \mu\right)}=\left\{\int_{0}^{\infty}\left(\mu\left(\left\{x \in \mathbb{R}_{+}^{n+1}:|g(x)|>s\right\}\right)\right)^{p / q} d s^{p}\right\}^{1 / p}<\infty
$$

and

$$
\|\left. g\right|_{L^{q}\left(\mathbb{R}_{+}^{n+1}, \mu\right)}=\left(\int_{\mathbb{R}_{+}^{n+1}}|g(x)|^{q} d \mu\right)^{1 / q}<\infty,
$$

respectively. Moreover, we denote by $L^{q, \infty}\left(\mathbb{R}_{+}^{n+1}, \mu\right)$ the set of all $\mu$-measurable functions $g$ on $\mathbb{R}_{+}^{n+1}$ with

$$
\|g\|_{L^{q, \infty}\left(\mathbb{R}_{+}^{n+1}, \mu\right)}=\sup _{s>0} s\left(\mu\left(\left\{x \in \mathbb{R}_{+}^{n+1}:|g(x)|>s\right\}\right)\right)^{1 / q}<\infty .
$$

The embedding (1.4) will be characterized by conditions in terms of capacities and Hedberg-Wolff potentials of $\mu$. Firstly, we introduce the $L^{p}$-capacity associated with the Caffarelli-Silvestre extension.

Definition 1.1. Let $1 \leq p<\infty$. For a subset $E$ of $\mathbb{R}_{+}^{n+1}$, let

$$
C_{\mathbb{R}_{+}^{n+1}}^{\alpha, p}(E):=\inf \left\{\|f\|_{L^{p}\left(\mathbb{R}^{n}\right)}^{p}: f \geq 0 \& P_{\alpha} f(x, t) \geq 1 \text { for all }(x, t) \in E\right\} .
$$

When the set $\left\{f \in L^{p}\left(\mathbb{R}^{n}\right): f \geq 0 \& P_{\alpha} f(x, t) \geq 1\right.$ for all $\left.(x, t) \in E\right\}$ is empty, we set $C_{\mathbb{R}_{+}^{n+1}}^{\alpha, p}(E)=0$.

Then, we establish the mixed norm estimate of $P_{\alpha} f(x, t)$, the dual form of $C_{\mathbb{R}_{+}^{n+1}}^{\alpha, p}(\cdot)$, some basic properties of $C_{\mathbb{R}_{+}^{n+1}}^{\alpha, p}(\cdot)$ and a capacitary strong type inequality.

Embedding II: Given $\alpha \in(0,2)$ and a nonnegative Radon measure $\mu$ on $\mathbb{R}_{+}^{n+1}$,

$$
\left\|P_{\alpha} f(\cdot, \cdot)\right\|_{L^{q}\left(\mathbb{R}_{+}^{n+1}, \mu\right)} \lesssim\|f\|_{\dot{W}^{\beta, p}\left(\mathbb{R}^{n}\right)}
$$

for $0<\beta<n, 1 \leq p \leq n / \beta$ and $1<q<\infty$.

We will show that the embedding (1.5) when $p=1$ can be characterized by conditions in terms of the fractional perimeter of open sets for the endpoint cases and fractional capacities for general cases. The fractional perimeter and the fractional capacity are defined, respectively, as follows. 
Definition 1.2. Let $s \in(0,1)$. The fractional perimeter is defined as

$$
\operatorname{Per}_{s}(E)=\int_{E} \int_{\mathbb{R}^{n} \backslash E} \frac{1}{|x-y|^{n+s}} d x d y
$$

for a given measurable set $E \subseteq \mathbb{R}^{n}$.

It follows from the definition of $\|f\|_{\dot{W}^{s, 1}\left(\mathbb{R}^{n}\right)}$ that $\operatorname{Per}_{s}(E)=\frac{1}{2}\left\|1_{E}\right\|_{\dot{W}^{s, 1}\left(\mathbb{R}^{n}\right)}$. On the other hand, there holds

$$
\operatorname{Per}_{s}(E)=\frac{\Gamma((n+s) / 2)}{2 \pi^{n / 2} \Gamma(s / 2)} \int_{\mathbb{R}_{+}^{n+1}}\left|\nabla u_{E}(x, t)\right|^{2} t^{1-s} d x d t,
$$

where $u_{E}(\cdot, \cdot)$ is the solution to equation (1.1) with $f=1_{E}$.

For the fractional perimeter, Ambrosio-DePhilippis-Martinazzi in [6] proved the generalized coarea formula:

$$
\|f\|_{\dot{W}^{s, 1}\left(\mathbb{R}^{n}\right)}=2 \int_{0}^{\infty} \operatorname{Per}_{s}(\{x: f(x)>t\}) d t
$$

for every nonnegative $f \in \dot{W}^{s, 1}\left(\mathbb{R}^{n}\right)$.

Denote by $T(O)$ the tent based on an open subset $O$ of $\mathbb{R}^{n}$ :

$$
T(O)=\left\{(x, r) \in \mathbb{R}_{+}^{n+1}: B(x, r) \subseteq O\right\}
$$

with $B(x, r)$ the open ball centered at $x \in \mathbb{R}^{n}$ with radius $r>0$.

Definition 1.3. Let $\beta \in(0, n)$ and $p \in[1, n / \beta]$.

(i) The fractional capacity of an arbitrary set $S \subset \mathbb{R}^{n}$, denoted by $C a p_{\mathbb{R}^{n}}^{\beta, p}(S)$, is defined as

$$
\operatorname{Cap}_{\mathbb{R}^{n}}^{\beta, p}(S):=\inf \left\{\|f\|_{\dot{W}^{\beta, p}\left(\mathbb{R}^{n}\right)}^{p}: f \in C_{0}^{\infty}\left(\mathbb{R}^{n}\right), f \geq 0 \text { on } \mathbb{R}^{n} \& f \geq 1_{S}\right\} .
$$

(ii) For $t \in(0, \infty)$, the $(p, \beta)$-fractional capacity minimizing function associated with both $\dot{W}^{\beta, p}\left(\mathbb{R}^{n}\right)$ and a nonnegative measure $\mu$ on $\mathbb{R}_{+}^{n+1}$, denoted by $c_{p}^{\beta}(\mu, t)$, is set as

$$
c_{p}^{\beta}(\mu, t):=\inf \left\{\operatorname{Cap}_{\mathbb{R}^{n}}^{\beta, p}(O): \text { bounded open } O \subseteq \mathbb{R}^{n}, \mu(T(O))>t\right\} .
$$

This article is mainly motivated by the work on embeddings like (1.4) and (1.5) via classical/fractional heat equations. Xiao in [28] studied the embeddings of the homogeneous Sobolev space $\dot{W}^{1, p}\left(\mathbb{R}^{n}\right)$ into the Lebesgue space $L^{q}\left(\mathbb{R}_{+}^{n+1}, \mu\right)$, under $(p, q) \in(1, \infty) \times \mathbb{R}_{+}$, via the Gauss-Weierstrass heat kernel. For fractional diffusion equations, motivated by Xiao [28], Zhai in [31] explored the embeddings of the homogeneous Sobolev space $\dot{W}^{\beta, p}\left(\mathbb{R}^{n}\right)$ into the Lebesgue space $L^{q}\left(\mathbb{R}_{+}^{n+1}, \mu\right)$. By using the $L^{p}$-capacities associated with the fractional heat kernel, Chang-Xiao in [13] and Shi-Xiao in [24] established embeddings similar to (1.4). For applications of Poisson like kernel in function spaces, see [9, 19] and the references therein.

This article will be organized as follows. In Section 2.1, we investigate the dual form and basic properties of the $L^{p}$-capacity $C_{\mathbb{R}_{+}^{n+1}}^{\alpha, p}(\cdot)$, and Section 2.2 is devoted to a capacitary strong type inequality corresponding to $C_{\mathbb{R}_{+}^{n+1}}^{\alpha, p}(\cdot)$. Section 2.3 describes several technical lemmas on the fractional capacity $\operatorname{Cap}_{\mathbb{R}^{n}}^{\beta, p}(\cdot)$. In Section 3 , we deduce the embedding (1.4) for two cases $p \leq q$ and $p>q$, respectively. Section 4 studies the embedding (1.5) for two cases $p \leq q$ and $p>q$.

Some notations: Let us agree to more conventions. $\mathrm{U} \approx \mathrm{V}$ represents that there is a constant $c>0$ such that $c^{-1} \mathrm{~V} \leq \mathrm{U} \leq c \mathrm{~V}$ whose right inequality is also written as $\mathrm{U} \lesssim \mathrm{V}$. Similarly, one writes $\mathrm{V} \gtrsim \mathrm{U}$ for $\mathrm{V} \geq c \mathrm{U}$. 


\section{Preliminaries on CAPacities}

2.1. $L^{p}$ - capacities associated with the Caffarelli-Silvestre extension. In this section, $\alpha \in(0,2)$. We will first establish the dual form of the $L^{p}$-capacity associated with the Caffarelli-Silvestre extension. Then, we will prove some basic properties of the $L^{p}$-capacity and the capacitary strong type inequality for $C_{\mathbb{R}_{+}^{n+1}}^{\alpha, p}(\cdot)$.

To establish the adjoint formulation of the foregoing definition, we need to find out the adjoint operator of $P_{\alpha}$. Note that

$$
\int_{\mathbb{R}_{+}^{n+1}} P_{\alpha} f(x, t) g(x, t) d t d x=\int_{\mathbb{R}^{n}} f(x)\left(\int_{\mathbb{R}_{+}^{n+1}} p_{t}^{\alpha}(x-z) g(z, t) d z d t\right) d x
$$

holds for all $f \in C_{0}^{\infty}\left(\mathbb{R}^{n}\right)$ and $g \in C_{0}^{\infty}\left(\mathbb{R}_{+}^{n+1}\right)$. The adjoint operator denoted by $P_{\alpha}^{*}$ can be defined as

$$
\left(P_{\alpha}^{*} g\right)(x):=\int_{\mathbb{R}_{+}^{n+1}} p_{t}^{\alpha}(x-z) g(z, t) d z d t, \quad g \in C_{0}^{\infty}\left(\mathbb{R}_{+}^{n+1}\right) .
$$

For a Borel measure $\mu$ with compact support in $\mathbb{R}_{+}^{n+1}$, we define

$$
P_{\alpha}^{*} \mu(x):=\int_{\mathbb{R}_{+}^{n+1}} p_{t}^{\alpha}(x-z) d \mu(z, t) .
$$

Proposition 2.1. Given $p \in(1, \infty)$ and a compact subset $K$ of $\mathbb{R}_{+}^{n+1}$, let $p^{\prime}=p /(p-1)$ and $\mathcal{M}_{+}(K)$ be the class of nonnegative Radon measures supported on $K$. Then

(i)

$$
C_{\mathbb{R}_{+}^{n+1}}^{\alpha, p}(K)=\sup \left\{\|\mu\|_{1}^{p}: \mu \in \mathcal{M}_{+}(K) \&\left\|P_{\alpha}^{*} \mu\right\|_{L^{p^{\prime}}\left(\mathbb{R}^{n}\right)} \leq 1\right\}
$$

(ii) There exists a measure $\mu_{K} \in \mathcal{M}_{+}(K)$ such that

$$
\mu_{K}(K)=\int_{\mathbb{R}^{n}}\left(P_{\alpha}^{*} \mu_{K}(x)\right)^{p^{\prime}} d x=\int_{\mathbb{R}_{+}^{n+1}} P_{\alpha}\left(P_{\alpha}^{*} \mu_{K}(x)\right)^{p^{\prime}-1} d \mu_{K}=C_{\mathbb{R}_{+}^{n+1}}^{\alpha, p}(K) .
$$

Proof. (i) We set

$$
{\widetilde{C^{\prime}}}_{\mathbb{R}_{+}^{n+1}}^{\alpha, p}:=\sup \left\{\|\mu\|_{1}^{p}: \mu \in \mathcal{M}_{+}(K) \&\left\|P_{\alpha}^{*} \mu\right\|_{L^{p^{\prime}}\left(\mathbb{R}^{n}\right)} \leq 1\right\} .
$$

Let $\mu \in \mathcal{M}_{+}(K)$ satisfy $\left\|P_{\alpha}^{*} \mu\right\|_{L^{p^{\prime}}\left(\mathbb{R}^{n}\right)} \leq 1$. Since $\|\mu\|_{1}=\mu(K)$, for any $f \geq 0$ and $P_{\alpha} f \geq 1_{K}$,

$$
\|\mu\|_{1} \leq \int_{\mathbb{R}_{+}^{n+1}} P_{\alpha} f d \mu=\int_{\mathbb{R}^{n}} f(x) P_{\alpha}^{*} \mu(x) d x \leq\|f\|_{L^{p}\left(\mathbb{R}^{n}\right)}\left\|P_{\alpha}^{*} \mu\right\|_{L^{p^{\prime}}\left(\mathbb{R}^{n}\right)} \leq\|f\|_{L^{p}\left(\mathbb{R}^{n}\right)}
$$

which implies $\widetilde{C}_{\mathbb{R}_{+}^{n+1}}^{\alpha, p}(K) \leq C_{\mathbb{R}_{+}^{n+1}}^{\alpha, p}(K)$.

Conversely, define

$$
\left\{\begin{array}{l}
\mathcal{X}:=\left\{\mu: \mu \in \mathcal{M}_{+}(K) \quad \& \quad \mu(K)=1\right\} \\
\mathcal{Y}:=\left\{f: 0<f \in L^{p}\left(\mathbb{R}^{n}\right) \quad \& \quad\|f\|_{L^{p}}\left(\mathbb{R}^{n}\right) \leq 1\right\} \\
\mathcal{Z}:=\left\{f: 0 \leq f \in L^{p}\left(\mathbb{R}^{n}\right) \quad \& \quad P_{\alpha} f \geq 1_{K}\right\} \\
E(\mu, f):=\int_{\mathbb{R}^{n}}\left(P_{\alpha}^{*} \mu\right)(x) f(x) d x=\int_{\mathbb{R}_{+}^{n+1}} P_{\alpha} f(x, t) d \mu(x, t) .
\end{array}\right.
$$

By [4, Thorem 2.4.1], $\min _{\mu \in X} \sup _{f \in \mathcal{Y}} E(\mu, f)=\sup _{f \in \mathcal{Y}} \min _{\mu \in X} E(\mu, f)$. We can get 


$$
\begin{aligned}
& \min _{\mu \in \mathcal{M}_{+}(K)} \frac{\left\|P_{\alpha}^{*} \mu\right\|_{L^{p^{\prime}}\left(\mathbb{R}^{n}\right)}}{\mu(K)}=\min _{\mu \in \mathcal{M}_{+}(K)} \frac{\sup _{0 \leq f \in L^{p}\left(\mathbb{R}^{n}\right) \&\|f\|_{L^{p}\left(\mathbb{R}^{n}\right)} \leq 1} \int_{\mathbb{R}^{n}} f(x) P_{\alpha}^{*} \mu(x) d x}{\mu(K)} \\
& =\min _{\mu \in \mathcal{M}_{+}(K)} \frac{\sup _{f \in L^{p}\left(\mathbb{R}^{n}\right) \&\|f\|_{L^{p}\left(\mathbb{R}^{n}\right)}=1} \int_{\mathbb{R}^{n}} f(x) P_{\alpha}^{*} \mu(x) d x}{\mu(K)} \\
& \leq \min _{\mu \in \mathcal{M}_{+}(K)} \sup _{f \in \mathcal{Y}} \frac{\int_{\mathbb{R}^{n}} f(x) P_{\alpha}^{*} \mu(x) d x}{\|f\|_{L^{p}\left(\mathbb{R}^{n}\right)} \mu(K)} \\
& =\sup _{f \in \mathcal{Y}} \min _{\mu \in \mathcal{X}} \frac{1}{\|f\|_{L^{p}\left(\mathbb{R}^{n}\right)}}\left\{\int_{K} P_{\alpha} f(x, t) d \mu(x, t)\right\} \\
& =\sup _{f \in \mathcal{Y}} \frac{1}{\|f\|_{L^{p}\left(\mathbb{R}^{n}\right)}}\left(\min _{(x, t) \in K} P_{\alpha} f(x, t)\right) \min _{\mu \in \mathcal{X}} \mu(K) \\
& \leq \sup _{0<f \in L^{p}\left(\mathbb{R}^{n}\right)} \frac{\left(\min _{(x, t) \in K} P_{\alpha} f(x, t)\right)}{\|f\|_{L^{p}\left(\mathbb{R}^{n}\right)}} \\
& \leq\left(\inf _{0 \leq f \in L^{p}\left(\mathbb{R}^{n}\right) \& \min P_{\alpha} f=1}\|f\|_{L^{p}\left(\mathbb{R}^{n}\right)}\right)^{-1} \\
& =\left(C_{\mathbb{R}_{+}^{n+1}}^{\alpha, p}(K)\right)^{-1 / p} \text {. }
\end{aligned}
$$

For any $\mu \in \mathcal{M}_{+}(K)$, take $\mu_{1}:=\left\|P_{\alpha}^{*} \mu\right\|_{L^{p^{\prime}\left(\mathbb{R}^{n}\right)}}^{-1} \mu$. It is obvious that $\left\|P_{\alpha}^{*} \mu_{1}\right\|_{L^{p^{\prime}}\left(\mathbb{R}^{n}\right)}=1$, and consequently,

$$
\left(\widetilde{C}_{\mathbb{R}_{+}^{n+1}}^{\alpha, p}(K)\right)^{1 / p} \geq \sup \left\{\frac{\|\mu\|_{1}}{\left\|P_{\alpha}^{*} \mu\right\|_{L^{p^{\prime}}\left(\mathbb{R}^{n}\right)}}: \mu \in \mathcal{M}_{+}(K)\right\}=\sup \left\{\left\|\mu_{1}\right\|_{1}, \mu_{1} \in \mathcal{M}_{+}(K)\right\}
$$

which implies

$$
\min _{\mu \in \mathcal{M}_{+}(K)}\left\{\frac{\left\|P_{\alpha}^{*} \mu\right\|_{L^{p^{\prime}\left(\mathbb{R}^{n}\right)}}}{\mu(K)}\right\}=\min _{\mu \in \mathcal{M}_{+}(K)}\left\{\frac{\left\|P_{\alpha}^{*} \mu\right\|_{L^{p^{\prime}}\left(\mathbb{R}^{n}\right)}}{\|\mu\|_{1}}\right\} \geq\left(\widetilde{C}_{\mathbb{R}_{+}^{\alpha+1}}^{\alpha, p}(K)\right)^{-1 / p}
$$

This gives $\left(C_{\mathbb{R}_{+}^{n+1}}^{\alpha, p}(K)\right)^{-1 / p} \geq\left(\widetilde{C}_{\mathbb{R}_{+}^{n+1}}^{\alpha, p}(K)\right)^{-1 / p}$. The proof of (i) is completed.

Next let us verify (ii). According to (i), we select a sequence $\left\{\mu_{j}\right\} \subset \mathcal{M}_{+}(K)$ such that

$$
\left\{\begin{array}{l}
\lim _{j \rightarrow \infty}\left(\mu_{j}(K)\right)^{p}=C_{\mathbb{R}_{+}^{n+1}}^{\alpha, p}(K) \\
\left\|P_{\alpha}^{*} \mu_{j}\right\|_{L^{p^{\prime}}\left(\mathbb{R}^{n}\right)} \leq 1
\end{array}\right.
$$

A direct computation implies that

$$
C_{\mathbb{R}_{+}^{n+1}}^{\alpha, p}(K)=\sup \left\{\|\mu\|_{1}^{p}: \mu \in \mathcal{M}_{+}(K) \&\left\|P_{\alpha}^{*} \mu\right\|_{L^{p^{\prime}\left(\mathbb{R}^{n}\right)}}=1\right\} .
$$

Then, using the fact $\left\|P_{\alpha}^{*} \mu_{j}\right\|_{L^{p^{\prime}\left(\mathbb{R}^{n}\right)}}=1$, we get

$$
\left|\int_{\mathbb{R}_{+}^{n+1}} P_{\alpha} f(x, t) d \mu_{j}(x, t)\right|=\left|\int_{\mathbb{R}^{n}} f(x) P_{\alpha}^{*} \mu_{j}(x) d x\right| \leq\left\|P_{\alpha}^{*} \mu_{j}\right\|_{L^{p^{\prime}}\left(\mathbb{R}^{n}\right)}\|f\|_{L^{p}\left(\mathbb{R}^{n}\right)} \leq\|f\|_{L^{p}\left(\mathbb{R}^{n}\right)} .
$$

There exists $\mu \in \mathcal{M}_{+}(K)$ such that $\mu_{j}$ weak $*$ convergence to $\mu$. Hence $\mu^{p}(K)=C_{\mathbb{R}_{+}^{n+1}}^{\alpha, p}(K)$. Since the Lebesgue space $L^{p}\left(\mathbb{R}^{n}\right)$ is uniformly convex for $1<p<\infty$, the set

$$
\left\{f \in C_{0}^{\infty}\left(\mathbb{R}^{n}\right): f \geq 0 \text { on } \mathbb{R}^{n} \text { and } f \geq 1\right\}
$$


is a convex set. Following the procedure of [4, Theorem 2.3.10], we can prove that there exists a unique function denoted by $f_{K}$ such that

$$
\left\{\begin{array}{l}
f_{K} \in L^{p}\left(\mathbb{R}^{n}\right) \\
C_{\mathbb{R}_{+}^{n+1}}^{\alpha, p}\left(\left\{(x, t) \in K: P_{\alpha} f_{K}<1\right\}\right)=0 \\
\left\|f_{K}\right\|_{L^{p}\left(\mathbb{R}^{n}\right)}^{p}=C_{\mathbb{R}_{+}^{n+1}}^{\alpha, p}(K)
\end{array}\right.
$$

Then we have

$$
\begin{aligned}
\left(C_{\mathbb{R}_{+}^{n+1}}^{\alpha, p}(K)\right)^{1 / p}=\mu(K) & \leq \int_{\mathbb{R}_{+}^{n+1}} P_{\alpha} f(x, t) d \mu(x, t) \\
& \leq \int_{\mathbb{R}^{n}} f_{K}(x) P_{\alpha}^{*} \mu(x) d x \\
& \leq\left\|f_{K}\right\|_{L^{p}\left(\mathbb{R}^{n}\right)}\left\|P_{\alpha}^{*} \mu\right\|_{L^{p^{\prime}}\left(\mathbb{R}^{n}\right)} \\
& =\left(C_{\mathbb{R}_{+}^{\alpha+1}}^{\alpha, p}(K)\right)^{1 / p}\left\|P_{\alpha}^{*} \mu\right\|_{L^{p^{\prime}}\left(\mathbb{R}^{n}\right)},
\end{aligned}
$$

which implies that $\left\|P_{\alpha}^{*} \mu\right\|_{L^{p^{\prime}\left(\mathbb{R}^{n}\right)}} \geq 1$. On the other hand, following the procedure of [4] Proposition 2.3.2, (b)], we can prove that $\mu \longmapsto P_{\alpha}^{*} \mu(x)$ is lower semi-continuous on $M_{+}\left(\mathbb{R}_{+}^{n+1}\right)$ in the weak* topology, i.e.,

$$
P_{\alpha}^{*} \mu(x) \leq \liminf _{j \rightarrow \infty} P_{\alpha}^{*} \mu_{j}(x),
$$

which, together with $\left\|P_{\alpha}^{*} \mu_{j}\right\|_{L^{p^{\prime}\left(\mathbb{R}^{n}\right)}} \leq 1$, gives $\left\|P_{\alpha}^{*} \mu\right\|_{L^{p^{\prime}}\left(\mathbb{R}^{n}\right)} \leq 1$. Hence $\left\|P_{\alpha}^{*} \mu\right\|_{L^{p^{\prime}\left(\mathbb{R}^{n}\right)}}=1$. Taking $\mu_{K}=$ $\left(C_{\mathbb{R}_{+}^{n+1}}^{\alpha, p}(K)\right)^{1 / p^{\prime}} \mu$ yields

$$
\begin{aligned}
\mu_{K}(K) & =\int_{K}\left(C_{\mathbb{R}_{+}^{n+1}}^{\alpha, p}(K)\right)^{1 / p^{\prime}} d \mu=\left(C_{\mathbb{R}_{+}^{n+1}}^{\alpha, p}(K)\right)^{1 / p^{\prime}} \mu(K) \\
& =\left(C_{\mathbb{R}_{+}^{n+1}}^{\alpha, p}(K)\right)^{1 / p^{\prime}}\left(C_{\mathbb{R}_{+}^{n+1}}^{\alpha, p}(K)\right)^{1 / p}=C_{\mathbb{R}_{+}^{\alpha+1}}^{\alpha, p}(K) .
\end{aligned}
$$

On the other hand,

$$
\int_{\mathbb{R}^{n}}\left(P_{\alpha}^{*} \mu_{K}(x)\right)^{p^{\prime}} d x=\left\|P_{\alpha}^{*} \mu_{K}\right\|_{L^{p^{\prime}\left(\mathbb{R}^{n}\right)}}^{p^{\prime}}=\left(C_{\mathbb{R}_{+}^{n+1}}^{\alpha, p}(K)\right)\left\|P_{\alpha}^{*} \mu\right\|_{L^{p^{\prime}}\left(\mathbb{R}^{n}\right)}^{p^{\prime}}=C_{\mathbb{R}_{+}^{n+1}}^{\alpha, p}(K)
$$

This indicates that

$$
\mu_{K}(K)=\int_{\mathbb{R}^{n}}\left(P_{\alpha}^{*} \mu_{K}(x)\right)^{p^{\prime}} d x=C_{\mathbb{R}_{+}^{n+1}}^{\alpha, p}(K)
$$

Let $f_{K}$ be the function mentioned above. Then $\mu_{K}\left(\left\{(x, t) \in K: P_{\alpha} f_{K}(x, t) \leq 1\right\}\right)=0$.

By Hölder's inequality, we can get

$$
\begin{aligned}
C_{\mathbb{R}_{+}^{n+1}}^{\alpha, p}(K) & =\mu_{K}(K) \leq \int_{K} P_{\alpha} f_{K} d \mu_{K} \\
& \leq \int_{\mathbb{R}^{n}} f_{K}(x) P_{\alpha}^{*} \mu_{K}(x) d x \\
& \leq\left\|f_{K}\right\|_{L^{p}\left(\mathbb{R}^{n}\right)}\left\|P_{\alpha}^{*} \mu_{K}\right\|_{L^{p^{\prime}}\left(\mathbb{R}^{n}\right)} \\
& =\left(C_{\mathbb{R}_{+}^{\alpha+1}}^{\alpha, p}(K)\right)^{1 / p}\left(C_{\mathbb{R}_{+}^{\alpha, p}}^{n+1}(K)\right)^{1 / p^{\prime}}=C_{\mathbb{R}_{+}^{n+1}}^{\alpha, p}(K) .
\end{aligned}
$$

It follows from (2.4) that

Hence

$$
C_{\mathbb{R}_{+}^{n+1}}^{\alpha, p}(K)=\int_{\mathbb{R}^{n}} f_{K} P_{\alpha}^{*} \mu_{K} d x
$$

$$
\int_{\mathbb{R}^{n}} f_{K} P_{\alpha}^{*} \mu_{K} d x=\int_{\mathbb{R}^{n}}\left(P_{\alpha}^{*} \mu_{K}\right)^{p^{\prime}} d x=C_{\mathbb{R}_{+}^{n+1}}^{\alpha, p}(K)
$$


The above identity implies that

$$
C_{\mathbb{R}_{+}^{n+1}}^{\alpha, p}(K)=\int_{\mathbb{R}_{+}^{n+1}} P_{\alpha} f_{K} d \mu_{K}=\int_{\mathbb{R}_{+}^{n+1}} P_{\alpha}\left(P_{\alpha}^{*} \mu_{K}\right)^{p^{\prime}-1} d \mu_{K},
$$

which completes the proof of Proposition 2.1

Remark 2.2. The inequality (2.4) implies that

$$
\int_{\mathbb{R}^{n}} f_{K}(x) P_{\alpha}^{*} \mu_{K}(x) d x=\left\|f_{K}\right\|_{L^{p}\left(\mathbb{R}^{n}\right)}\left\|P_{\alpha}^{*} \mu_{K}\right\|_{L^{p^{\prime}}\left(\mathbb{R}^{n}\right)},
$$

i.e., we have equality in Hölder inequality. This means that, due to the normalization chosen, $\left(f_{K}\right)^{p}=$ $\left(P_{\alpha}^{*} \mu_{K}\right)^{p^{\prime}}$.

Below we investigate some basic properties of $C_{\mathbb{R}_{+}^{n+1}}^{\alpha, p}(\cdot)$.

\section{Proposition 2.3.}

(i) $C_{\mathbb{R}_{+}^{n+1}}^{\alpha, p}(\varnothing)=0$;

(ii) If $E_{1} \subseteq E_{2} \subset \mathbb{R}_{+}^{n+1}$, then $C_{\mathbb{R}_{+}^{n+1}}^{\alpha, p}\left(E_{1}\right) \leq C_{\mathbb{R}_{+}^{n+1}}^{\alpha, p}\left(E_{2}\right)$;

(iii) For any sequence $\left\{E_{j}\right\}_{j=1}^{\infty}$ of subsets of $\mathbb{R}_{+}^{n+1}$,

$$
C_{\mathbb{R}_{+}^{n+1}}^{\alpha, p}\left(\bigcup_{j=1}^{\infty} E_{j}\right) \leq \sum_{j=1}^{\infty} C_{\mathbb{R}_{+}^{n+1}}^{\alpha, p}\left(E_{j}\right) ;
$$

(iv) For any $E \subset \mathbb{R}_{+}^{n+1}$ and any $x_{0} \in \mathbb{R}^{n}, C_{\mathbb{R}_{+}^{n+1}}^{\alpha, p}\left(E+\left(x_{0}, 0\right)\right)=C_{\mathbb{R}_{+}^{n+1}}^{\alpha, p}(E)$.

Proof. The statements (i) \& (ii) can be deduced from the definition of $C_{\mathbb{R}_{+}^{n+1}}^{\alpha, p}(\cdot)$ immediately. For (iii), let $\epsilon>0$. Take $f_{j} \geq 0$ such that $P_{\alpha} f_{j} \geq 1$ on $E_{j}$ and $\int_{\mathbb{R}^{n}}\left|f_{j}(x)\right|^{p} d x \leq C_{\mathbb{R}_{+}^{n+1}}^{\alpha, p}\left(E_{j}\right)+\epsilon / 2^{j}$. Let $f=\sup _{j \in \mathbb{N}_{+}} f_{j}$. For any $(x, t) \in \cup_{j=1}^{\infty} E_{j}$, there exists a $j_{0}$ such that $(x, t) \in E_{j_{0}}$ and $P_{\alpha} f_{j_{0}}(x, t) \geq 1$. Hence $P_{\alpha} f(x, t) \geq 1$ on $\cup_{j=1}^{\infty} E_{j}$. On the other hand,

$$
\|f\|_{L^{p}\left(\mathbb{R}^{n}\right)}^{p}=\int_{\mathbb{R}^{n}}|f(x)|^{p} d x \leq \sum_{j=1}^{\infty} \int_{\mathbb{R}^{n}}\left|f_{j}(x)\right|^{p} d x=\sum_{j=1}^{\infty} C_{\mathbb{R}_{+}^{n+1}}^{\alpha, p}\left(E_{j}\right)+\epsilon,
$$

which indicates $C_{\mathbb{R}_{+}^{n+1}}^{\alpha, p}\left(\cup_{j=1}^{\infty} E_{j}\right) \leq \sum_{j=1}^{\infty} C_{\mathbb{R}_{+}^{n+1}}^{\alpha, p}\left(E_{j}\right)$.

Now we verify (iv). Define $f_{x_{0}}(x)=f\left(x+x_{0}\right)$. Then $\|f\|_{L^{p}\left(\mathbb{R}^{n}\right)}=\left\|f_{x_{0}}\right\|_{L^{p}\left(\mathbb{R}^{n}\right)}$. If $(x, t) \in E+\left(x_{0}, 0\right)$, then $\left(x-x_{0}, t\right) \in E$ and vice versa. Take $f \geq 0$ such that $P_{\alpha} f \geq 1_{E}$. Changing of variables reaches

$$
P_{\alpha} f_{x_{0}}(x, t)=\int_{\mathbb{R}^{n}} p_{t}^{\alpha}(x-y) f_{x_{0}}(y) d y=P_{\alpha} f\left(x-x_{0}, t\right),
$$

which implies that $P_{\alpha} f_{x_{0}}(x, t) \geq 1_{E+\left(x_{0}, 0\right)}$ is equivalent to $P_{\alpha} f\left(x-x_{0}, t\right) \geq 1_{E}$. This gives $C_{\mathbb{R}_{+}^{n+1}}^{\alpha, p}(E+$ $\left.\left(x_{0}, 0\right)\right)=C_{\mathbb{R}_{+}^{n+1}}^{\alpha, p}(E)$ and the proof of Proposition 2.3 is completed.

We then prove that the capacity $C_{\mathbb{R}_{+}^{n+1}}^{\alpha, p}(\cdot)$ is an outer capacity, i.e.,

Proposition 2.4. For any $E \subset \mathbb{R}_{+}^{n+1}$,

$$
C_{\mathbb{R}_{+}^{n+1}}^{\alpha, p}(E)=\inf \left\{C_{\mathbb{R}_{+}^{n+1}}^{\alpha, p}(O): O \supset E, O \text { open }\right\} .
$$


Proof. Without loss of generality, we assume that $C_{\mathbb{R}_{+}^{n+1}}^{\alpha, p}(E)<\infty$. By (ii) of Proposition 2.3 ,

$$
C_{\mathbb{R}_{+}^{n+1}}^{\alpha, p}(E) \leq \inf \left\{C_{\mathbb{R}_{+}^{n+1}}^{\alpha, p}(O): O \supset E, O \text { open }\right\} .
$$

For $\epsilon \in(0,1)$, there exists a measurable, nonnegative function $f$ such that $P_{\alpha} f \geq 1$ on $E$ and

$$
\int_{\mathbb{R}^{n}}|f(x)|^{p} d x \leq C_{\mathbb{R}_{+}^{n+1}}^{\alpha, p}(E)+\epsilon .
$$

Since $P_{\alpha} f$ is lower semi-continuous, then the set $O_{\epsilon}:=\left\{(x, t) \in \mathbb{R}_{+}^{n+1}: P_{\alpha} f(x, t)>1-\epsilon\right\}$ is an open set. On the other hand, $E \subset O_{\epsilon}$. This implies that

$$
C_{\mathbb{R}_{+}^{n+1}}^{\alpha, p}\left(O_{\epsilon}\right) \leq \frac{1}{(1-\epsilon)^{p}} \int_{\mathbb{R}^{n}}|f(x)|^{p} d x<\frac{1}{(1-\epsilon)^{p}}\left(C_{\mathbb{R}_{+}^{n+1}}^{\alpha, p}(E)+\epsilon\right) .
$$

The arbitrariness of $\epsilon$ indicates that

$$
C_{\mathbb{R}_{+}^{n+1}}^{\alpha, p}(E) \geq \inf \left\{C_{\mathbb{R}_{+}^{n+1}}^{\alpha, p}(O): O \supset E, O \text { open }\right\} .
$$

An immediate corollary of Proposition 2.4 is the follow result.

Corollary 2.5. If $\left\{K_{j}\right\}_{j=1}^{\infty}$ is a decreasing sequence of compact sets, then

$$
C_{\mathbb{R}_{+}^{n+1}}^{\alpha, p}\left(\cap_{j=1}^{\infty} K_{j}\right)=\lim _{j \rightarrow \infty} C_{\mathbb{R}_{+}^{n+1}}^{\alpha, p}\left(K_{j}\right) .
$$

Proof. Let $O$ be any open set satisfying $\cap_{j=1}^{\infty} K_{j} \subset O$. Then for some $j, K_{j} \subset O$, which, together with Proposition 2.4, gives

$$
C_{\mathbb{R}_{+}^{n+1}}^{\alpha, p}\left(\cap_{j=1}^{\infty} K_{j}\right) \leq \lim _{j \rightarrow \infty} C_{\mathbb{R}_{+}^{n+1}}^{\alpha, p}\left(K_{j}\right) \leq \inf _{\cap_{j=1}^{\infty} K_{j} \subset O} C_{\mathbb{R}_{+}^{n+1}}^{\alpha, p}(O)=C_{\mathbb{R}_{+}^{n+1}}^{\alpha, p}\left(\cap_{j=1}^{\infty} K_{j}\right) .
$$

Proposition 2.6. Let $1<p<\infty$. If $\left\{E_{j}\right\}_{j=1}^{\infty}$ is an increasing sequence of arbitrary subsets of $\mathbb{R}^{n}$, then

$$
C_{\mathbb{R}_{+}^{n+1}}^{\alpha, p}\left(\cup_{j=1}^{\infty} E_{j}\right)=\lim _{j \rightarrow \infty} C_{\mathbb{R}_{+}^{n+1}}^{\alpha, p}\left(E_{j}\right) .
$$

Proof. Since $\left\{E_{j}\right\}_{j=1}^{\infty}$ is increasing, then

$$
C_{\mathbb{R}_{+}^{n+1}}^{\alpha, p}\left(\cup_{j=1}^{\infty} E_{j}\right) \geq \lim _{j \rightarrow \infty} C_{\mathbb{R}_{+}^{n+1}}^{\alpha, p}\left(E_{j}\right) .
$$

Conversely, without loss generality, we assume that $\lim _{j \rightarrow \infty} C_{\mathbb{R}_{+}^{n+1}}^{\alpha, p}\left(E_{j}\right)$ is finite. For each $j$, let $f_{E_{j}}$ be the unique function such that $f_{E_{j}} \geq 1$ on $E_{j}$ and $\left\|f_{E_{j}}\right\|_{L^{p}}^{p}=C_{\mathbb{R}_{+}^{n+1}}^{\alpha, p}\left(E_{j}\right)$. Then for $i<j$, it holds that $P_{\alpha} f_{E_{j}} \geq 1$ on $E_{i}$ and further, $P_{\alpha}\left(\left(f_{E_{i}}+f_{E_{j}}\right) / 2\right) \geq 1$ on $E_{i}$, which means that

$$
\int_{\mathbb{R}^{n}}\left(\left(f_{E_{i}}+f_{E_{j}}\right) / 2\right)^{p} d x \geq C_{\mathbb{R}_{+}^{n+1}}^{\alpha, p}\left(E_{i}\right)
$$

By [4, Corollary 1.3.3], the sequence $\left\{f_{E_{j}}\right\}_{j=1}^{\infty}$ converges strongly to a function $f$ satisfying

$$
\|f\|_{L^{p}\left(\mathbb{R}^{n}\right)}^{p}=\lim _{j \rightarrow \infty} C_{\mathbb{R}_{+}^{n+1}}^{\alpha, p}\left(E_{j}\right) .
$$

Similar to [4, Proposition 2.3.12], we can prove that $P_{\alpha} f \geq 1$ on $\cup_{j=1}^{\infty} E_{j}$, except possibly on a countable union of sets with $C_{\mathbb{R}_{+}^{n+1}}^{\alpha, p}(\cdot)$ zero. Hence

$$
\lim _{j \rightarrow \infty} C_{\mathbb{R}_{+}^{n+1}}^{\alpha, p}\left(E_{j}\right) \geq \int_{\mathbb{R}^{n}}|f(x)|^{p} d x \geq C_{\mathbb{R}_{+}^{n+1}}^{\alpha, p}\left(\cup_{j=1}^{\infty} E_{j}\right) .
$$


As a corollary of Proposition 2.6, we can get

Corollary 2.7. Let $O$ be an open subset of $\mathbb{R}_{+}^{n+1}$. Then

$$
C_{\mathbb{R}_{+}^{n+1}}^{\alpha, p}(O)=\sup \left\{C_{\mathbb{R}_{+}^{n+1}}^{\alpha, p}(K): \text { compact } K \subset O\right\} .
$$

Proof. It is obvious that

$$
C_{\mathbb{R}_{+}^{n+1}}^{\alpha, p}(O) \geq \sup \left\{C_{\mathbb{R}_{+}^{n+1}}^{\alpha, p}(K): \text { compact } K \subset O\right\} .
$$

Conversely, any open set $O$ in $\mathbb{R}^{n}$ is the union of an increasing sequence of compact sets denoted by $\left\{K_{j}\right\}_{j=1}^{\infty}$. Then it follows from Proposition 2.6 that

$$
\begin{aligned}
C_{\mathbb{R}_{+}^{n+1}}^{\alpha, p}(O) & =C_{\mathbb{R}_{+}^{n+1}}^{\alpha, p}\left(\cup_{j=1}^{\infty} K_{j}\right)=\lim _{j \rightarrow \infty} C_{\mathbb{R}_{+}^{n+1}}^{\alpha, p}\left(K_{j}\right) \\
& \leq \sup \left\{C_{\mathbb{R}_{+}^{n+1}}^{\alpha, p}(K): \text { compact } K \subset O\right\} .
\end{aligned}
$$

For $t_{0} \geq 0, x_{0} \in \mathbb{R}^{n}$ and $r_{0}>0$, define the ball in $\mathbb{R}_{+}^{n+1}$ as

$$
B_{r_{0}}\left(x_{0}, t_{0}\right)=\left\{(x, t) \in \mathbb{R}_{+}^{n+1}:\left|x-x_{0}\right|<r_{0} / 2, r_{0}<t-t_{0}<2 r_{0}\right\} .
$$

Let $t=r_{0} s, x=r_{0} y$ and $f_{r_{0}}(x)=f\left(r_{0} x\right)$. We can get $(x, t) \in B_{r_{0}}(0,0)$ is equivalent to $(y, s) \in B_{1}(0,0)$. A direct computation, together with a change of variable $z=r_{0} u$, gives

$$
\begin{aligned}
P_{\alpha} f(x, t) & =\int_{\mathbb{R}^{n}} \frac{t^{\alpha}}{\left(t^{2}+|x-z|^{2}\right)^{(n+\alpha) / 2}} f(z) d z \\
& =\int_{\mathbb{R}^{n}} \frac{s^{\alpha}}{\left(|y-u|^{2}+s^{2}\right)^{(n+\alpha) / 2}} f_{r_{0}}(u) d u=P_{\alpha} f_{r_{0}}(y, r) .
\end{aligned}
$$

Then

$$
P_{\alpha} f(x, t) \geq 1 \quad \forall(x, t) \in B_{r_{0}}(0,0) \Longleftrightarrow P_{\alpha} f_{r_{0}}(s, y) \geq 1 \quad \forall(y, s) \in B_{1}(0,0) .
$$

This means that

$$
C_{\mathbb{R}_{+}^{n+1}}^{\alpha, p}\left(B_{r_{0}}(0,0)\right)=r_{0}^{n} C_{\mathbb{R}_{+}^{n+1}}^{\alpha, p}\left(B_{1}(0,0)\right) .
$$

Now we investigate the $L^{p}$-capacity of general balls $B_{r_{0}}\left(x_{0}, t_{0}\right)$. We first give a space-time estimate for $P_{\alpha} f$.

Lemma 2.8. Let $1 \leq r \leq p \leq \infty$. Then $\left\|P_{\alpha} f(\cdot, t)\right\|_{L^{p}\left(\mathbb{R}^{n}\right)} \leq t^{n(1 / p-1 / r)}\|f\|_{L^{r}\left(\mathbb{R}^{n}\right)}$.

Proof. Let $q$ obey $1 / p+1=1 / r+1 / q$. It is easy to see that

$$
\left\|p_{t}^{\alpha}(\cdot)\right\|_{L^{q}\left(\mathbb{R}^{n}\right)}=\left(\int_{\mathbb{R}^{n}}\left|\frac{t^{\alpha}}{\left(t^{2}+|x|^{2}\right)^{(n+\alpha) / 2}}\right|^{q} d x\right)^{1 / q} \lesssim t^{n(1 / q-1)} .
$$

It follows from Young's inequality that

$$
\left\|P_{\alpha} f(\cdot, t)\right\|_{L^{p}\left(\mathbb{R}^{n}\right)} \leq\left\|p_{t}^{\alpha} * f\right\|_{L^{p}\left(\mathbb{R}^{n}\right)} \leq\left\|p_{t}^{\alpha}(\cdot)\right\|_{L^{q}\left(\mathbb{R}^{n}\right)}\|f\|_{L^{r}\left(\mathbb{R}^{n}\right)} \leq t^{n(1 / p-1 / r)}\|f\|_{L^{r}\left(\mathbb{R}^{n}\right)} .
$$

Theorem 2.9. Let $(q, p, r)$ be a triple satisfying $1 / q=n(1 / r-1 / p)$, where

$$
1<r \leq p<\left\{\begin{array}{l}
n r /(n-1), n>2 r ; \\
\infty, n \leq 2 r .
\end{array}\right.
$$

Given $f \in L^{r}\left(\mathbb{R}^{n}\right)$. Then for $I=(0, T)$ with $0<T \leq \infty, P_{\alpha} f(\cdot, \cdot) \in L^{q}\left(I ; L^{p}\left(\mathbb{R}^{n}\right)\right) \cap C_{b}\left(I ; L^{r}\left(\mathbb{R}^{n}\right)\right)$ with the estimate

$$
\left\|P_{\alpha} f(\cdot, \cdot)\right\|_{L^{q}\left(I ; L^{p}\left(\mathbb{R}^{n}\right)\right)} \lesssim\|f\|_{L^{r}\left(\mathbb{R}^{n}\right)} .
$$

Here $C_{b}\left(I ; L^{r}\left(\mathbb{R}^{n}\right)\right)$ denotes the space of bounded continuous functions from I to $L^{r}\left(\mathbb{R}^{n}\right)$. 
Proof. Case 1: $p=r \& q=\infty$. By Lemma2.8, we can get

$$
\left\|P_{\alpha} f\right\|_{L^{\infty}\left(I ; L^{r}\left(\mathbb{R}^{n}\right)\right)}=\sup _{t>0}\left\|p_{t}^{\alpha} * f\right\|_{L^{r}\left(\mathbb{R}^{n}\right)} \leq \sup _{t>0} t^{-n(1 / r-1 / r)}\|f\|_{L^{r}\left(\mathbb{R}^{n}\right)} \leq\|f\|_{L^{r}\left(\mathbb{R}^{n}\right)} .
$$

Case 2: $p \neq r$. Denote by $F_{t}(f)=\left\|p_{t}^{\alpha} * f\right\|_{L^{p}\left(\mathbb{R}^{n}\right)}$. Applying Lemma 2.8 again, we also obtain

$$
F_{t}(f)=\left\|p_{t}^{\alpha} * f\right\|_{L^{p}\left(\mathbb{R}^{n}\right)} \leq t^{-n(1 / r-1 / p)}\|f\|_{L^{r}\left(\mathbb{R}^{n}\right)} \leq t^{-1 / q}\|f\|_{L^{r}\left(\mathbb{R}^{n}\right)} .
$$

On the other hand,

$$
\left|\left\{t:\left|F_{t}(f)\right|>\tau\right\}\right| \leq\left|\left\{t: t^{-1 / q}\|f\|_{L^{r}\left(\mathbb{R}^{n}\right)}>\tau\right\}\right| \leq\left|\left\{t:\|f\|_{L^{r}\left(\mathbb{R}^{n}\right)}^{q} / \tau^{q}>t\right\}\right| \leq\|f\|_{L^{r}\left(\mathbb{R}^{n}\right)}^{q} / \tau^{q} .
$$

The above estimate implies that $F_{t}$ is a weak $(r, q)$ type operator. Noticing that

$$
\left|p_{t}^{\alpha} * f(x)\right| \leq \int_{\mathbb{R}^{n}} \frac{t^{\alpha}}{\left(t^{2}+|x-y|^{2}\right)^{(n+\alpha) / 2}}|f(y)| d y,
$$

we have

$$
\left|F_{t}(f)\right|=\left\|P_{\alpha} f(\cdot, t)\right\|_{L^{p}\left(\mathbb{R}^{n}\right)} \leq t^{n(1 / p-1 / p)}\|f\|_{L^{p}\left(\mathbb{R}^{n}\right)},
$$

i.e., $F_{t}$ is also a $(p, \infty)$ type operator. For any triplet $(q, p, r)$, we can choose another triplet $\left(q_{1}, p_{1}, r_{1}\right)$ such that $q_{1}<q<\infty, r_{1}<r<p$ and

$$
\left\{\begin{array}{l}
1 / q=\theta / q_{1}+(1-\theta) / \infty \\
1 / r=\theta / r_{1}+(1-\theta) / p
\end{array}\right.
$$

The Marcinkiewicz interpolation theorem implies that $F_{t}$ is a strong $(r, q)$ type operator and satisfies

$$
\left\|P_{\alpha} f(\cdot, \cdot)\right\|_{L^{q}\left(I ; L^{p}\left(\mathbb{R}^{n}\right)\right)} \leq\|f\|_{L^{r}\left(\mathbb{R}^{n}\right)} .
$$

Below we prove that $P_{\alpha} f(\cdot, \cdot) \in C_{b}\left(I ; L^{r}\left(\mathbb{R}^{n}\right)\right)$. Since $u(\cdot, \cdot):=P_{\alpha} f(\cdot, \cdot)$ satisfies the equations 1.1. Then by a direct computation, we can verify that $u(\cdot, \cdot)$ also satisfies:

$$
\begin{cases}\Delta_{x} u+\frac{1-\alpha}{t} u_{t}+u_{t t}=0, & (x, t) \in \mathbb{R}^{n} \times(0, \infty) ; \\ u(x, 0)=f(x), & x \in \mathbb{R}^{n},\end{cases}
$$

see [11]. Then by the Poisson type formula obtained by P. Stinga and J. Torrea (cf. [26, (1.9)]),

$$
u(x, t) \equiv P_{\alpha} f(x)=C_{\alpha} \int_{0}^{\infty} e^{-s} e^{-t^{2}(-\Delta) / 4 s} f(x) s^{\alpha / 2-1} d s, \quad x \in \mathbb{R}^{n} \& t>0 .
$$

By Minkowski's inequality, we get

$$
\begin{aligned}
\left|\|u(\cdot, t)\|_{L^{r}\left(\mathbb{R}^{n}\right)}-\left\|u\left(\cdot, t_{0}\right)\right\|_{L^{r}\left(\mathbb{R}^{n}\right)}\right| & \leq\left\|u(\cdot, t)-u\left(\cdot, t_{0}\right)\right\|_{L^{r}\left(\mathbb{R}^{n}\right)} \\
& \leq C_{\alpha} \int_{0}^{\infty} e^{-s}\left\|e^{-t^{2}(-\Delta) / 4 s} f(\cdot)-e^{-t_{0}^{2}(-\Delta) / 4 s} f(\cdot)\right\|_{L^{r}\left(\mathbb{R}^{n}\right)} s^{\alpha / 2-1} d s .
\end{aligned}
$$

Notice that

$$
\left\|e^{-t^{2}(-\Delta) / 4 s} f(\cdot)-e^{-t_{0}^{2}(-\Delta) / 4 s} f(\cdot)\right\|_{L^{r}\left(\mathbb{R}^{n}\right)} \leq C\|f\|_{L^{r}\left(\mathbb{R}^{n}\right)} .
$$

Since $\left\{e^{-t(-\Delta)}\right\}_{t>0}$ is a strongly continuous semigroups in $L^{r}\left(\mathbb{R}^{n}\right)$, then by the dominate convergence theorem, we can deduce that

$$
\lim _{t \rightarrow t_{0}}\|u(\cdot, t)\|_{L^{r}\left(\mathbb{R}^{n}\right)}=\left\|u\left(\cdot, t_{0}\right)\right\|_{L^{r}\left(\mathbb{R}^{n}\right)} .
$$

For $p \in(1, \infty)$, choose $(\tilde{p}, \tilde{q})$ such that

$$
\left\{\begin{array}{l}
1 \leq p \leq \tilde{p}<n p /(n-1) \\
1 / \tilde{q}=n(1 / p-1 / \tilde{p})
\end{array}\right.
$$


Theorem 2.10. If $1 \leq p<\infty$, then for $\left(x_{0}, r_{0}\right) \in \mathbb{R}_{+}^{n+1}$,

$$
r_{0}^{n} \lesssim C_{\mathbb{R}_{+}^{n+1}}^{\alpha, p}\left(B_{r_{0}}\left(x_{0}, t_{0}\right)\right) \lesssim\left(t_{0}+r_{0}\right)^{p n} r_{0}^{n(1-p)} .
$$

Particularly, if $t_{0} \lesssim r_{0}$, then

$$
r_{0}^{n} \lesssim C_{\mathbb{R}_{+}^{\alpha+1}}^{\alpha, p}\left(B_{r_{0}}\left(x_{0}, t_{0}\right)\right) \lesssim r_{0}^{n} .
$$

Proof. Let $B_{r_{0}}\left(x_{0}, t_{0}\right)$ be a ball in $\mathbb{R}_{+}^{n+1}$. For any $f \geq 0$ satisfying $P_{\alpha}(f) \geq 1_{B_{r_{0}}\left(x_{0}, t_{0}\right)}$, by (2.5), we can get

$$
r_{0}^{n / \tilde{p}+1 / \tilde{q}} \lesssim\left(\int_{r_{0}<t-t_{0}<2 r_{0}}\left(\int_{\left|x-x_{0}\right|<r_{0} / 2}\left|P_{\alpha} f(x, t)\right|^{\tilde{p}} d x\right)^{\tilde{q} / \tilde{p}} d t\right)^{1 / \tilde{q}} \lesssim\|f\|_{L^{p}\left(\mathbb{R}^{n}\right)},
$$

which gives $r_{0}^{(n / \tilde{p}+1 / \tilde{q}) p} \leq\|f\|_{L^{p}\left(\mathbb{R}^{n}\right)}^{p}$ and $r_{0}^{n} \leq C_{\mathbb{R}_{+}^{n+1}}^{\alpha, p}\left(B_{r_{0}}\left(x_{0}, t_{0}\right)\right)$.

For the converse, choose $f=1_{\left\{x \in \mathbb{R}^{n}:\left|x-x_{0}\right|<r_{0} / 2\right\}}$. If $(x, t) \in B_{r_{0}}\left(x_{0}, t_{0}\right)$, then $\left|x-x_{0}\right|<r_{0} / 2$ and $r_{0}<t-t_{0}<2 r_{0}$, i.e., $r_{0}+t_{0} \leq t<t_{0}+2 r_{0}$. We can get there exists a constant $c_{\alpha}$ such that

$$
\frac{t^{\alpha}}{\left(t^{2}+|x-y|^{2}\right)^{(n+\alpha) / 2}} \geq \frac{\left(t_{0}+r_{0}\right)^{\alpha}}{\left(t_{0}+2 r_{0}+3 r_{0} / 2\right)^{(n+\alpha) / 2}} \geq \frac{c_{\alpha}}{\left(t_{0}+r_{0}\right)^{n}}
$$

which gives

$$
P_{\alpha} f(x, t)=\int_{\left|y-x_{0}\right|<r_{0} / 2} \frac{t^{\alpha}}{\left(t^{2}+|x-y|^{2}\right)^{(n+\alpha) / 2}} d y \geq \frac{c_{\alpha} r_{0}^{n}}{\left(t_{0}+r_{0}\right)^{n}},
$$

equivalently, $P_{\alpha}\left(\left(1+t_{0} / r_{0}\right)^{n} c_{\alpha}^{-1} f\right) \geq 1$. By the definition of $C_{\mathbb{R}_{+}^{n+1}}^{\alpha, p}(\cdot)$, we obtain

$$
\begin{aligned}
C_{\mathbb{R}_{+}^{n+1}}^{\alpha, p}\left(B_{r_{0}}\left(x_{0}, t_{0}\right)\right) & \leq\left\|\frac{\left(t_{0}+r_{0}\right)^{n}}{r_{0}^{n}} f\right\|_{L^{p}\left(\mathbb{R}^{n}\right)}^{p} \\
& =\frac{\left(t_{0}+r_{0}\right)^{p n}}{r_{0}^{p n}} \int_{\mathbb{R}^{n}}\left(1_{\left\{x \in \mathbb{R}^{n}:\left|x-x_{0}\right|<r_{0} / 2\right\}}(y)\right)^{p} d y \\
& =\left(t_{0}+r_{0}\right)^{p n} r_{0}^{(1-p) n},
\end{aligned}
$$

which completes the proof of Theorem 2.10 ,

2.2. Capacitary strong type inequality. In order to establish the embeddings of $L^{p}\left(\mathbb{R}^{n}\right)$ to $L^{q}\left(\mathbb{R}_{+}^{n+1}\right)$ via $P_{\alpha}$, we need to prove the capacitary strong and weak inequalities. Let $L_{+}^{p}\left(\mathbb{R}^{n}\right)$ denote the class of all nonnegative functions in $L^{p}\left(\mathbb{R}^{n}\right)$.

Lemma 2.11. Let $p \in(1, \infty)$. Then

$$
\int_{0}^{\infty} C_{\mathbb{R}_{+}^{n+1}}^{\alpha, p}\left(\left\{(x, t) \in \mathbb{R}_{+}^{n+1}: P_{\alpha} f(x, t) \geq \lambda\right\}\right) d \lambda^{p} \lesssim\|f\|_{L^{p}\left(\mathbb{R}^{n}\right)}^{p} \quad \forall f \in L_{+}^{p}\left(\mathbb{R}^{n}\right) .
$$

Proof. Firstly, we verify the inequality for any nonnegative function in $C_{0}^{\infty}\left(\mathbb{R}^{n}\right)$. Let $f \in C_{0}^{\infty}\left(\mathbb{R}^{n}\right)$ and for $r>0$, set

$$
E_{j}:=\left\{(x, t) \in \overline{B_{r}(0,0)}: P_{\alpha} f(x, t) \geq 2^{j}\right\} .
$$

Note that $E_{j}$ depends on $r$. Since $f \in C_{0}^{\infty}\left(\mathbb{R}^{n}\right), P_{\alpha} f(x, t)$ is continuous. Thus, $E_{j}$ is the intersection of $\overline{B_{r}(0,0)}$ and a close set and so $E_{j}$ is compact.

Let $\mu_{j}$ stand for the measure corresponding to $E_{j}$ such that

$$
\mu_{j}\left(E_{j}\right)=\int_{\mathbb{R}^{n}}\left(P_{\alpha}^{*} \mu_{j}(x)\right)^{p^{\prime}} d x=\int_{\mathbb{R}_{+}^{n+1}} P_{\alpha}\left(P_{\alpha}^{*} \mu_{j}\right)^{p^{\prime}-1} d \mu_{j}=C_{\mathbb{R}_{+}^{n+1}}^{\alpha, p}\left(E_{j}\right) .
$$


Let $S:=\sum_{j=-\infty}^{\infty} 2^{j p} \mu_{j}\left(E_{j}\right)$ and $T:=\left\|\sum_{j=-\infty}^{\infty} 2^{j(p-1)}\left(P_{\alpha}^{*} \mu_{j}\right)\right\|_{L^{p^{\prime}}}^{p^{\prime}}$. Because $f \in C_{0}^{\infty}\left(\mathbb{R}^{n}\right)$, there exists a positive integer $j_{0}$ such that $E_{j}$ are empty sets for $j>j_{0}$, i.e., $\mu_{j}\left(E_{j}\right)=C_{\mathbb{R}_{+}^{n+1}}^{\alpha, p}\left(E_{j}\right)=0, j>j_{0}$. Hence $\sum_{j=1}^{\infty} 2^{j p} \mu_{j}\left(E_{j}\right)<\infty$. On the other hand,

$$
\begin{aligned}
\sum_{j=\infty}^{-1} 2^{j p} \mu_{j}\left(E_{j}\right) & =\sum_{j=\infty}^{-1} 2^{j p} C_{\mathbb{R}_{+}^{n+1}}^{\alpha, p}\left(E_{j}\right) \\
& \leq \sum_{j=\infty}^{-1} 2^{j p} C_{\mathbb{R}_{+}^{\alpha+1}}^{\alpha, p}\left(B_{r}(0,0)\right) \\
& \lesssim r^{n} \sum_{j=\infty}^{-1} 2^{j p},
\end{aligned}
$$

which means that $S<\infty$. Then by Hölder's inequality,

$$
S \leq \sum_{j=-\infty}^{\infty} 2^{j(p-1)} \int_{\mathbb{R}_{+}^{n+1}} P_{\alpha} f d \mu_{j} \leq \int_{\mathbb{R}^{n}} f\left(\sum_{j=-\infty}^{\infty} 2^{j(p-1)}\left(P_{\alpha}^{*} \mu_{j}\right)\right) d x \leq T^{1 / p^{\prime}}\|f\|_{L^{p}\left(\mathbb{R}^{n}\right)} .
$$

Below we claim that

$$
T \lesssim S .
$$

We divide the proof of this claim into two cases.

Case 1: $2 \leq p<\infty$. For $k=0, \pm 1, \pm 2, \ldots$, let

$$
\left\{\begin{array}{l}
\sigma_{k}(x)=\sum_{j=k}^{\infty} 2^{j(p-1)} P_{\alpha}^{*} \mu_{j}(x) ; \\
\sigma(x)=\sum_{j=-\infty}^{\infty} 2^{j(p-1)} P_{\alpha}^{*} \mu_{j}(x) .
\end{array}\right.
$$

Since $f \in C_{0}^{\infty}\left(\mathbb{R}^{n}\right)$, the sets $E_{j}$ are empty for sufficiently large $j$, i.e., there exists a positive integer $j_{0}$ such that $C_{\mathbb{R}_{+}^{n+1}}^{\alpha, p}\left(E_{j}\right)=0, j>j_{0}$. Then by (ii) of Proposition 2.1,

$$
\begin{aligned}
\left\|\sigma_{k}\right\|_{L^{p^{\prime}\left(\mathbb{R}^{n}\right)}} & =\left\|\sum_{j=k}^{\infty} 2^{j(p-1)} P_{\alpha}^{*} \mu_{j}\right\|_{L^{p^{\prime}\left(\mathbb{R}^{n}\right)}} \\
& \leq \sum_{j=k}^{\infty} 2^{j(p-1)}\left\|P_{\alpha}^{*} \mu_{j}\right\|_{L^{p^{\prime}\left(\mathbb{R}^{n}\right)}} \\
& \simeq \sum_{j=k}^{j_{0}} 2^{j(p-1)}\left(C_{\mathbb{R}_{+}^{\alpha, p}}^{\alpha, p}\left(E_{j}\right)\right)^{1 / p^{\prime}}<\infty,
\end{aligned}
$$

which implies that for any $k, \sigma_{k} \in L^{p^{\prime}}\left(\mathbb{R}^{n}\right)$. For $x \in \mathbb{R}^{n}$, the sequence $\left\{\sigma_{k}(x)\right\}$ is increasing as $k \rightarrow-\infty$ since $P_{\alpha}^{*} \mu_{j}(x) \geq 0$. If there exists an upper bound for $\left\{\sigma_{k}(x)\right\}$, we know that $\sigma_{k}(x)$ convergences to the series $\sum_{j=-\infty}^{\infty} 2^{j(p-1)} P_{\alpha}^{*} \mu_{j}(x)$. If the sequence $\left\{\sigma_{k}(x)\right\}$ is unbounded, then $\sigma_{k}(x)$ tends to $\infty$ as $k \rightarrow-\infty$. Without loss of generality, formally, we write $\lim _{k \rightarrow-\infty} \sigma_{k}(x)=\sigma(x)$. We can get

$$
\sum_{k=n}^{\infty}\left(\sigma_{k}(x)^{p^{\prime}}-\sigma_{k+1}(x)^{p^{\prime}}\right)=\sum_{k=n}^{\infty} \sigma_{k}(x)^{p^{\prime}}-\sum_{k=n+1}^{\infty} \sigma_{k}(x)^{p^{\prime}}=\sigma_{n}(x)^{p^{\prime}} .
$$


By the mean value theorem, noticing that $\sigma_{k}(x) \geq \sigma_{k+1}(x)$, we have

$$
\begin{aligned}
\sigma(x)^{p^{\prime}} & =\left(\sum_{j=-\infty}^{\infty} 2^{j(p-1)} P_{\alpha}^{*} \mu_{j}(x)\right)^{p^{\prime}}=p^{\prime} \sum_{k=-\infty}^{\infty}\left(\sigma_{k}(x)\right)^{p^{\prime}-1} \\
& =\lim _{n \rightarrow-\infty} \sigma_{n}(x)^{p^{\prime}} \\
& =\lim _{n \rightarrow-\infty} \sum_{k=n}^{\infty}\left(\sigma_{k}(x)^{p^{\prime}}-\sigma_{k+1}(x)^{p^{\prime}}\right) \\
& \leq p^{\prime}\left(\sigma_{k}(x)\right)^{p^{\prime}-1}\left(\sigma_{k}(x)-\sigma_{k+1}(x)\right) \\
& =p^{\prime} \sum_{k=-\infty}^{\infty}\left(\sigma_{k}(x)\right)^{p^{\prime}-1} 2^{k(p-1)} P_{\alpha}^{*} \mu_{k}(x)
\end{aligned}
$$

Using Cauchy-Schwartz's inequality, we obtain

$$
\begin{aligned}
T= & p^{\prime} \int_{\mathbb{R}^{n}} \sum_{k=-\infty}^{\infty} \sigma_{k}^{p^{\prime}-1}(x) 2^{k(p-1)} P_{\alpha}^{*} \mu_{k}(x) d x \\
\lesssim & p^{\prime} \int_{\mathbb{R}^{n}}\left(\sum_{k=-\infty}^{\infty}\left(\sigma_{k}(x)\right) 2^{k} \frac{1}{(p-1)} \frac{1}{p^{\prime}-1}\left(P_{\alpha}^{*} \mu_{k}\right)^{p^{\prime}-1}\right)^{p^{\prime}-1} \\
& \times\left(\sum_{k=-\infty}^{\infty} 2^{k(p-1) \frac{p(p-2)}{(p-1)^{2}} \frac{1}{2-p^{\prime}}}\left(P_{\alpha}^{*} \mu_{k}(x)\right)^{p^{\prime}}\right)^{2-p^{\prime}} d x \\
= & p^{\prime} \int_{\mathbb{R}^{n}}\left(\sum_{k=-\infty}^{\infty} \sigma_{k}(x) 2^{k}\left(P_{\alpha}^{*} \mu_{k}\right)^{p^{\prime}-1}\right)^{p^{\prime}-1}\left(\sum_{k=-\infty}^{\infty} 2^{k p}\left(P_{\alpha}^{*} \mu_{k}(x)\right)^{p^{\prime}}\right)^{2-p^{\prime}} d x,
\end{aligned}
$$

which, together with Hölder's inequality, indicates that $T \lesssim p^{\prime} T_{1}^{2-p^{\prime}} T_{2}^{p^{\prime}-1}$, where

$$
\left\{\begin{array}{l}
T_{1}:=\int_{\mathbb{R}^{n}}\left(\sum_{k=-\infty}^{\infty} 2^{k p}\left(P_{\alpha}^{*} \mu_{k}(x)\right)^{p^{\prime}}\right) d x \\
T_{2}:=\int_{\mathbb{R}^{n}}\left(\sum_{k=-\infty}^{\infty} \sigma_{k}(x) 2^{k}\left(P_{\alpha}^{*} \mu_{k}\right)^{p^{\prime}-1}\right) d x .
\end{array}\right.
$$

For $T_{1}$, we have

$$
\begin{aligned}
T_{1} & =\sum_{k=-\infty}^{\infty} 2^{k p} \int_{\mathbb{R}^{n}}\left(P_{\alpha}^{*} \mu_{k}(x)\right)^{p^{\prime}} d x=\sum_{k=-\infty}^{\infty} 2^{k p} C_{\mathbb{R}_{+}^{\alpha+1}}^{\alpha, p}\left(E_{k}\right) \\
& \lesssim \sum_{k=-\infty}^{\infty} \int_{2^{k-1}}^{2^{k}} C_{\mathbb{R}_{+}^{n+1}}^{\alpha, p}\left(\left\{(x, t) \in \overline{B_{r}(0,0)}: P_{\alpha} f(x, t) \geq 2^{k}\right\}\right) d \lambda^{p} \\
& \lesssim \sum_{k=-\infty}^{\infty} \int_{2^{k-1}}^{2^{k}} C_{\mathbb{R}_{+}^{n+1}}^{\alpha, p}\left(\left\{(x, t) \in \overline{B_{r}(0,0)}: P_{\alpha} f(x, t) \geq \lambda\right\}\right) d \lambda^{p} \\
& \lesssim \int_{0}^{\infty} C_{\mathbb{R}_{+}^{n+1}}^{\alpha, p}\left(\left\{(x, t) \in \overline{B_{r}(0,0)}: P_{\alpha} f(x, t) \geq \lambda\right\}\right) d \lambda^{p} \lesssim S .
\end{aligned}
$$


For $T_{2}$, we can get

$$
\begin{aligned}
T_{2} & =\sum_{k=-\infty}^{\infty} 2^{k} \int_{\mathbb{R}^{n}}\left(\sum_{j \geq k}^{\infty} 2^{j(p-1)} P_{\alpha}^{*} \mu_{j}(x)\right)\left(P_{\alpha}^{*} \mu_{k}\right)^{p^{\prime}-1} d x \\
& =\sum_{k=-\infty}^{\infty} \sum_{j \geq k}^{\infty} 2^{k+j(p-1)} \int_{\mathbb{R}^{n}} P_{\alpha}^{*} \mu_{j}(x)\left(P_{\alpha}^{*} \mu_{k}\right)^{p^{\prime}-1} d x \\
& =\sum_{k=-\infty}^{\infty} \sum_{j \geq k}^{\infty} 2^{k+j(p-1)} \int_{\mathbb{R}_{+}^{n+1}} P_{\alpha}\left(P_{\alpha}^{*} \mu_{k}\right)^{p^{\prime}-1} d \mu_{j}(x) .
\end{aligned}
$$

Note that $E_{k}$ is compact subsets. Let $f_{E_{k}}$ be the function satisfying 2.11. Suppose that $P_{\alpha} f_{E_{k}}\left(x_{0}, t_{0}\right)>1$, by the lower-semicontinuity, $P_{\alpha} f_{E_{j}} \geq 1+\delta>1$ on some neighborhood $U$ of $\left(x_{0}, t_{0}\right)$. On the other hand, denote by $\Omega_{E_{k}}$ the set $\left\{(x, t) \in E_{k}: P_{\alpha} f_{E_{k}}(x, t)<1\right\}$ and let $F$ be any compact subset of $\Omega_{E_{k}}$. For any $0 \leq f \in L^{p}\left(\mathbb{R}^{n}\right)$ such that $P_{\alpha} f \geq 1$ on $F$

$$
\begin{aligned}
\mu_{k}(F) & \leq \int_{\mathbb{R}_{+}^{n+1}} P_{\alpha} f d \mu_{k} \\
& =\int_{\mathbb{R}^{n}} f P_{\alpha}^{*} \mu_{k} d x \\
& \leq\left\|P_{\alpha}^{*} \mu_{k}\right\|_{L^{p^{\prime}}\left(\mathbb{R}^{n}\right)}\|f\|_{L^{p}\left(\mathbb{R}^{n}\right)},
\end{aligned}
$$

which, together with (2.1), gives

$$
\mu_{k}(F) \leq\left\|P_{\alpha}^{*} \mu_{k}\right\|_{L^{p^{\prime}\left(\mathbb{R}^{n}\right)}} C_{\mathbb{R}_{+}^{n+1}}^{\alpha, p}(F) \leq\left\|P_{\alpha}^{*} \mu_{k}\right\|_{L^{p^{\prime}}\left(\mathbb{R}^{n}\right)} C_{\mathbb{R}_{+}^{n+1}}^{\alpha, p}\left(\Omega_{E_{k}}\right)=0 .
$$

Hence, $\mu_{k}\left(\Omega_{E_{k}}\right)=0$, i.e., $P_{\alpha} f_{E_{k}} \geq 1$ on $E_{k}$ for $\mu_{k}$ a.e. We can deduce that

$$
\begin{aligned}
C_{\mathbb{R}_{+}^{n+1}}^{\alpha, p}\left(E_{k}\right) & =\int_{\mathbb{R}_{+}^{n+1}} P_{\alpha}\left(P_{\alpha}^{*} \mu_{k}\right)^{p^{\prime}-1} d \mu_{k} \\
& =\int_{\mathbb{R}_{+}^{n+1}} P_{\alpha} f_{E_{k}} d \mu_{k} \\
& \geq(1+\delta) \mu_{k}(U)+\mu_{k}\left(E_{k} \backslash U\right) \\
& \geq \delta \mu_{k}(U)+\mu_{k}\left(E_{k}\right),
\end{aligned}
$$

which gives $\mu_{k}(U)=0$, i.e., $\left(x_{0}, t_{0}\right) \notin \operatorname{supp} \mu_{k}$. Equivalently, for all $(x, t) \in \operatorname{supp} \mu_{k}$,

$$
P_{\alpha}\left(P_{\alpha}^{*} \mu_{k}\right)^{p^{\prime}-1}=P_{\alpha} f_{E_{k}} \leq 1 .
$$

Since $E_{j} \subset E_{k}$ for $j \geq k$, we obtain

$$
\begin{aligned}
T_{2} & \lesssim \sum_{k=-\infty}^{\infty} \sum_{j \geq k}^{\infty} 2^{k+j(p-1)} C_{\mathbb{R}_{+}^{n+1}}^{\alpha, p}\left(E_{j}\right) \\
& =\sum_{j=-\infty}^{\infty} 2^{j(p-1)} C_{\mathbb{R}_{+}^{n+1}}^{\alpha, p}\left(E_{j}\right) \sum_{k=-\infty}^{j} 2^{k} \\
& \lesssim \sum_{j=-\infty}^{\infty} 2^{j p} C_{\mathbb{R}_{+}^{n+1}}^{\alpha, p}\left(E_{j}\right) \lesssim S .
\end{aligned}
$$

The estimates for $T_{1}$ and $T_{2}$ imply (2.9). It can be deduced from (2.8) \& (2.9) that

$$
\left\|\sum_{j=-\infty}^{\infty} 2^{j(p-1)}\left(P_{\alpha}^{*} \mu_{j}\right)\right\|_{L^{p^{\prime}\left(\mathbb{R}^{n}\right)}}^{p^{\prime}} \lesssim\|f\|_{L^{p}\left(\mathbb{R}^{n}\right)}^{p},
$$


i.e., $\sigma \in L^{p^{\prime}}\left(\mathbb{R}^{n}\right)$ and the series $\sum_{j=-\infty}^{\infty} 2^{j(p-1)} P_{\alpha}^{*} \mu_{j}(x)<\infty$ a.e. $x \in \mathbb{R}^{n}$.

Case 2: $1<p<2$. For $k=0, \pm 1, \pm 2, \ldots$, let

$$
\left\{\begin{array}{l}
\sigma_{k}(x)=\sum_{j=-\infty}^{k} 2^{j(p-1)} P_{\alpha}^{*} \mu_{j}(x) ; \\
\sigma(x)=\sum_{j=-\infty}^{\infty} 2^{j(p-1)} P_{\alpha}^{*} \mu_{j}(x) .
\end{array}\right.
$$

Similarly, because $\sigma_{k}(x) \geq 0$ for $x \in \mathbb{R}^{n}$, we can also write $\lim _{k \rightarrow \infty} \sigma(x)=\sigma(x)$ formally. Below, similar to Case 1, we will prove (2.9) and $\sigma \in L^{p^{\prime}}\left(\mathbb{R}^{n}\right)$. Following the procedure of (2.10), we can deduce that

$$
(\sigma(x))^{p^{\prime}}=\left(\sum_{j=-\infty}^{\infty} 2^{j(p-1)} P_{\alpha}^{*} \mu_{j}(x)\right)^{p^{\prime}} \leq p^{\prime} \sum_{k=-\infty}^{\infty}\left(\sigma_{k}(x)\right)^{p^{\prime}-1} 2^{k(p-1)} P_{\alpha}^{*} \mu_{k}(x) .
$$

We get

$$
\begin{aligned}
T & =p^{\prime} \sum_{k=-\infty}^{\infty} 2^{k(p-1)} \int_{\mathbb{R}^{n}} P_{\alpha}^{*} \mu_{k}(x)\left(\sum_{j=-\infty}^{k} 2^{j(p-1)} P_{\alpha}^{*} \mu_{j}(x)\right)^{p^{\prime}-1} d x \\
& =p^{\prime} \sum_{k=-\infty}^{\infty} 2^{k(p-1)} \int_{\mathbb{R}^{n}}\left(\sum_{j=-\infty}^{k} 2^{j(p-1)}\left(P_{\alpha}^{*} \mu_{k}(x)\right)^{1 /\left(p^{\prime}-1\right)} P_{\alpha}^{*} \mu_{j}(x)\right)^{p^{\prime}-1} d x \\
& =p^{\prime} \sum_{k=-\infty}^{\infty} 2^{k(p-1)}\left\|\sum_{j=-\infty}^{k} 2^{j(p-1)}\left(P_{\alpha}^{*} \mu_{k}(x)\right)^{1 /\left(p^{\prime}-1\right)} P_{\alpha}^{*} \mu_{j}(x)\right\|_{L^{p^{\prime}-1}\left(\mathbb{R}^{n}\right)}^{p^{\prime}-1} \\
& \lesssim p^{\prime} \sum_{k=-\infty}^{\infty} 2^{k(p-1)}\left\{\sum_{j=-\infty}^{k} 2^{j(p-1)}\left\|\left(P_{\alpha}^{*} \mu_{k}(x)\right)^{1 /\left(p^{\prime}-1\right)} P_{\alpha}^{*} \mu_{j}(x)\right\|_{L^{p^{\prime}-1}\left(\mathbb{R}^{n}\right)}\right\}^{p^{\prime}-} .
\end{aligned}
$$

Also, for $j \leq k$, if $x \in E_{k}$ then $x \in E_{j}$. Similarly, we can deduce that

$$
P_{\alpha}\left(P_{\alpha}^{*} \mu_{j}(x)\right)^{p^{\prime}-1}(x) \leq 1, x \in E_{k} .
$$

This indicates that

$$
\begin{aligned}
\int_{\mathbb{R}^{n}}\left(P_{\alpha}^{*} \mu_{k}(x)\right)\left(P_{\alpha}^{*} \mu_{j}(x)\right)^{p^{\prime}-1} d x & =\int_{\mathbb{R}_{+}^{n+1}} P_{\alpha}\left(P_{\alpha}^{*} \mu_{j}(x)\right)^{p^{\prime}-1} d \mu_{k} \\
& \leq \mu_{k}\left(E_{k}\right)=C_{\mathbb{R}_{+}^{\alpha+1}}^{\alpha, p}\left(E_{k}\right) .
\end{aligned}
$$

We obtain

$$
\begin{aligned}
T & \lesssim \sum_{k=-\infty}^{\infty} 2^{k(p-1)}\left\{\sum_{j=-\infty}^{k} 2^{j(p-1)}\left(\int_{\mathbb{R}^{n}} P_{\alpha}^{*} \mu_{k}(x)\left(P_{\alpha}^{*} \mu_{j}(x)\right)^{p^{\prime}-1} d x\right)^{1 /\left(p^{\prime}-1\right)}\right\}^{p^{\prime}-1} \\
& \lesssim \sum_{k=-\infty}^{\infty} 2^{k(p-1)} C_{\mathbb{R}_{+}^{\alpha+1}}^{\alpha, p}\left(E_{k}\right)\left(\sum_{j=-\infty}^{k} 2^{j(p-1)}\right)^{p^{\prime}-1} \\
& \lesssim \sum_{k=-\infty}^{\infty} 2^{k p} C_{\mathbb{R}_{+}^{\alpha, p}}^{\alpha, p}\left(E_{k}\right) \lesssim S
\end{aligned}
$$

which gives 2.9) for $1<p<2$. 
It follows from (2.8) \& (2.9) that

$$
S \lesssim\|f\|_{L^{p}\left(\mathbb{R}^{n}\right)} T^{1 / p^{\prime}} \lesssim\|f\|_{L^{p}\left(\mathbb{R}^{n}\right)} S^{1 / p^{\prime}} .
$$

We can get $S \lesssim\|f\|_{L^{p}\left(\mathbb{R}^{n}\right)}^{p}$ and consequently, $T<\infty$. Then $\sigma \in L^{p^{\prime}}\left(\mathbb{R}^{n}\right)$, for $1<p<2$. By the fact that $f \in C_{0}^{\infty}\left(\mathbb{R}^{n}\right)$, there exists an integer $j_{0}$ such that

$$
\begin{aligned}
\left\|\sum_{j=0}^{\infty} 2^{j(p-1)} P_{\alpha}^{*} \mu_{j}\right\|_{L^{p^{\prime}\left(\mathbb{R}^{n}\right)}} & \leq \sum_{j=0}^{\infty} 2^{j(p-1)}\left\|P_{\alpha}^{*} \mu_{j}\right\|_{L^{p^{\prime}}\left(\mathbb{R}^{n}\right)} \\
& \simeq \sum_{j=0}^{j_{0}} 2^{j(p-1)}\left(C_{\mathbb{R}_{+}^{n+1}}^{\alpha, p}\left(E_{j}\right)\right)^{1 / p^{\prime}}<\infty .
\end{aligned}
$$

This indicates that $\sum_{j=-\infty}^{-1} 2^{j(p-1)} P_{\alpha}^{*} \mu_{j} \in L^{p^{\prime}}\left(\mathbb{R}^{n}\right)$. For $\sigma_{k}$, if $k \leq-1$, because $P_{\alpha}^{*} \mu_{j}(x) \geq 0$, then

$$
\left\|\sigma_{k}\right\|_{L^{p^{\prime}\left(\mathbb{R}^{n}\right)}} \leq\left\|\sum_{j=-\infty}^{-1} 2^{j(p-1)} P_{\alpha}^{*} \mu_{j}\right\|_{L^{p^{\prime}\left(\mathbb{R}^{n}\right)}}
$$

and $\sigma_{k} \in L^{p^{\prime}}\left(\mathbb{R}^{n}\right)$. If $k \geq 0$, noticing that

$$
\sigma_{k}=\sum_{j=-\infty}^{-1} 2^{j(p-1)} P_{\alpha}^{*} \mu_{j}+\sum_{j=0}^{j_{0}} 2^{j(p-1)} P_{\alpha}^{*} \mu_{j},
$$

we can also get $\sigma_{k} \in L^{p}\left(\mathbb{R}^{n}\right)$.

Further, we obtain that

$$
\begin{aligned}
& \int_{0}^{\infty} C_{\mathbb{R}_{+}^{n+1}}^{\alpha, p}\left(\left\{(x, t) \in \overline{B_{r}(0,0)}: P_{\alpha} f(x, t) \geq \lambda\right\}\right) d \lambda^{p} \\
& =\sum_{j=-\infty}^{\infty} \int_{2^{j}}^{2^{j+1}} C_{\mathbb{R}_{+}^{n+1}}^{\alpha, p}\left(\left\{(x, t) \in \overline{B_{r}(0,0)}: P_{\alpha} f(x, t) \geq \lambda\right\}\right) d \lambda^{p} \\
& \quad \lesssim \sum_{j=-\infty}^{\infty} C_{\mathbb{R}_{+}^{n+1}}^{\alpha, p}\left(E_{j}\right) \int_{2^{j}}^{2^{j+1}} d \lambda^{p} \\
& \quad \lesssim \sum_{j=-\infty}^{\infty} 2^{p j} \mu_{j}\left(E_{j}\right) \lesssim\|f\|_{L^{p}\left(\mathbb{R}^{n}\right)}^{p}
\end{aligned}
$$

Hence we can deduce from Proposition 2.6 and Fauto's lemma that

$$
\begin{aligned}
\int_{0}^{\infty} & C_{\mathbb{R}_{+}^{n+1}}^{\alpha, p}\left(\left\{(x, t) \in \mathbb{R}_{+}^{n+1}: P_{\alpha} f(x, t)>\lambda\right\}\right) d \lambda^{p} \\
= & \int_{0}^{\infty} C_{\mathbb{R}_{+}^{n+1}}^{\alpha, p}\left(\cup_{r=1}^{\infty}\left\{(x, t) \in \overline{B_{r}(0,0)}: P_{\alpha} f(x, t)>\lambda\right\}\right) d \lambda^{p} \\
= & \int_{0}^{\infty} \lim _{r \rightarrow \infty} C_{\mathbb{R}_{+}^{n+1}}^{\alpha, p}\left(\left\{(x, t) \in \overline{B_{r}(0,0)}: P_{\alpha} f(x, t)>\lambda\right\}\right) d \lambda^{p} \\
\leq & \underline{\lim }_{r \rightarrow \infty} \int_{0}^{\infty} C_{\mathbb{R}_{+}^{n+1}}^{\alpha, p}\left(\left\{(x, t) \in \overline{B_{r}(0,0)}: P_{\alpha} f(x, t)>\lambda\right\}\right) d \lambda^{p} \\
& \leq \underline{\lim }_{r \rightarrow \infty} \int_{0}^{\infty} C_{\mathbb{R}_{+}^{n+1}}^{\alpha, p}\left(\left\{(x, t) \in \overline{B_{r}(0,0)}: P_{\alpha} f(x, t) \geq \lambda\right\}\right) d \lambda^{p} \\
& \lesssim\|f\|_{L^{p}\left(\mathbb{R}^{n}\right)}^{p}
\end{aligned}
$$


which proves 2.77 for the functions in $C_{0}^{\infty}\left(\mathbb{R}^{n}\right)$.

At last, we prove that 2.77 holds for the functions in $L_{+}^{p}\left(\mathbb{R}^{n}\right)$. Let $f \in L_{+}^{p}\left(\mathbb{R}^{n}\right)$. Since $C_{0}^{\infty}\left(\mathbb{R}^{n}\right)$ is dense in $L_{+}^{p}\left(\mathbb{R}^{n}\right)$, there exists a sequence of $\left\{f_{j}\right\}_{j=1}^{\infty}$ such that $f_{n} \rightarrow f$ in $L^{p}\left(\mathbb{R}^{n}\right)$ as $n \rightarrow \infty$. Then it is obvious that $\left\|f_{j}\right\|_{L^{p}\left(\mathbb{R}^{n}\right)} \rightarrow\|f\|_{L^{p}\left(\mathbb{R}^{n}\right)}$ as $n \rightarrow \infty$. We can select a subsequence denoted by $\left\{f_{i_{j}}\right\}$ such that $\left\|f_{i_{j}}-f\right\|_{L^{p}\left(\mathbb{R}^{n}\right)}<4^{-j}$. For any $\lambda>0$, there exists an $m_{1} \in \mathbb{N}$ such that for $j \geq m_{1}$,

$$
\begin{aligned}
C_{\mathbb{R}_{+}^{n+1}}^{\alpha, p}\left(\left\{(x, t): P_{\alpha}\left(\left|f_{i_{j}}-f\right|\right)(x, t)>\lambda / 2\right\}\right) & \leq C_{\mathbb{R}_{+}^{n+1}}^{\alpha, p}\left(\left\{(x, t): P_{\alpha}\left(\left|f_{i_{j}}-f\right|\right)(x, t)>2^{-j}\right\}\right) \\
& \lesssim 2^{j p}\left\|f_{i_{j}}-f\right\|_{L^{p}\left(\mathbb{R}^{n}\right)}^{p} \\
& \lesssim 2^{-j p} .
\end{aligned}
$$

On the other hand, there exists an $m_{2} \in \mathbb{N}$ such that

$$
2^{-j p} \leq \frac{1}{2} C_{\mathbb{R}_{+}^{n+1}}^{\alpha, p}\left(\left\{(x, t): P_{\alpha}\left(\left|f_{i_{j}}-f\right|\right)(x, t)>\lambda\right\}\right) .
$$

Take $m:=\max \left\{m_{1}, m_{2}\right\}$. Then

$$
\begin{aligned}
C_{\mathbb{R}_{+}^{n+1}}^{\alpha, p}\left(\left\{(x, t): P_{\alpha}(f)(x, t)>\lambda\right\}\right) \leq & C_{\mathbb{R}_{+}^{n+1}}^{\alpha, p}\left(\left\{(x, t): P_{\alpha}\left(f_{i_{j}}\right)(x, t)>\lambda / 2\right\}\right) \\
& +C_{\mathbb{R}_{+}^{\alpha+1}}^{\alpha, p}\left(\left\{(x, t): P_{\alpha}\left(\left|f_{i_{j}}-f\right|\right)(x, t)>\lambda / 2\right\}\right) \\
\leq & C_{\mathbb{R}_{+}^{n+1}}^{\alpha, p}\left(\left\{(x, t): P_{\alpha}\left(f_{i_{j}}\right)(x, t)>\lambda / 2\right\}\right) \\
& +\frac{1}{2} C_{\mathbb{R}_{+}^{n+1}}^{\alpha, p}\left(\left\{(x, t): P_{\alpha}\left(\left|f_{i_{j}}-f\right|\right)(x, t)>\lambda\right\}\right),
\end{aligned}
$$

which means that $\forall j \geq m$,

$$
C_{\mathbb{R}_{+}^{n+1}}^{\alpha, p}\left(\left\{(x, t):\left|P_{\alpha}(f)\right|(x, t)>\lambda\right\}\right) \leq 2 C_{\mathbb{R}_{+}^{n+1}}^{\alpha, p}\left(\left\{(x, t):\left|P_{\alpha}\left(f_{i_{j}}\right)\right|(x, t)>\lambda / 2\right\}\right) .
$$

Then

$$
C_{\mathbb{R}_{+}^{n+1}}^{\alpha, p}\left(\left\{(x, t):\left|P_{\alpha}(f)\right|(x, t)>\lambda\right\}\right) \leq 2 \liminf _{j \rightarrow \infty} C_{\mathbb{R}_{+}^{n+1}}^{\alpha, p}\left(\left\{(x, t):\left|P_{\alpha}\left(f_{i_{j}}\right)\right|(x, t)>\lambda / 2\right\}\right) .
$$

By Fauto's lemma, we can get

$$
\begin{aligned}
& \int_{0}^{\infty} C_{\mathbb{R}_{+}^{n+1}}^{\alpha, p}\left(\left\{(x, t) \in \mathbb{R}_{+}^{n+1}: P_{\alpha} f(x, t) \geq \lambda\right\}\right) d \lambda^{p} \\
& \lesssim \int_{0}^{\infty} \liminf _{j \rightarrow \infty} C_{\mathbb{R}_{+}^{n+1}}^{\alpha, p}\left(\left\{(x, t):\left|P_{\alpha}\left(f_{j}\right)\right|(x, t)>\lambda / 2\right\}\right) d \lambda^{p} \\
& \lesssim \liminf _{j \rightarrow \infty} \int_{0}^{\infty} C_{\mathbb{R}_{+}^{n+1}}^{\alpha, p}\left(\left\{(x, t): P_{\alpha}\left(f_{i_{j}}\right)(x, t)>\lambda / 2\right\}\right) d \lambda^{p} \\
& \lesssim \liminf _{j \rightarrow \infty}\left\|f_{i_{j}}\right\|_{L^{p}\left(\mathbb{R}^{n}\right)}^{p} \\
& \lesssim\|f\|_{L^{p}\left(\mathbb{R}^{n}\right)^{p}}^{p} .
\end{aligned}
$$

2.3. Preliminary lemmas on the fractional capacity. Let $\mathcal{M}_{+}\left(\mathbb{R}_{+}^{n+1}\right)$ represent the class of all nonnegative Radon measures on $\mathbb{R}_{+}^{n+1}$.

Lemma 2.12. Let $\alpha \in(0,2)$ and $\beta \in(0, n)$. Given $f \in \dot{W}^{\beta, p}\left(\mathbb{R}^{n}\right), s>0$, and $\mu \in \mathcal{M}_{+}\left(\mathbb{R}_{+}^{n+1}\right)$, let

$$
L_{s}^{\alpha, \beta}(f)=\left\{(x, t) \in \mathbb{R}_{+}^{n+1}:\left|P_{\alpha} f(x, t)\right|>s\right\}
$$

and

$$
R_{s}^{\alpha, \beta}(f)=\left\{y \in \mathbb{R}^{n}: \sup _{|y-x|<t}\left|P_{\alpha} f(x, t)\right|>s\right\} .
$$

Then the following four statements are true. 
(i) For any natural number $k$

$$
\mu\left(L_{s}^{\alpha, \beta}(f) \cap T(B(0, k))\right) \leq \mu\left(T\left(R_{s}^{\alpha, \beta}(f) \cap B(0, k)\right)\right) .
$$

(ii) For any natural number $k$,

$$
C a p_{\mathbb{R}^{n}}^{\beta, p}\left(R_{s}^{\alpha, \beta}(f) \cap B(0, k)\right) \geq c_{p}^{\beta}\left(\mu, \mu\left(T\left(R_{s}^{\alpha, \beta}(f) \cap B(0, k)\right)\right) .\right.
$$

(iii) There exists a constant $\theta_{\alpha}>0$ such that

$$
\sup _{|y-x|<t}\left|P_{\alpha} f(y, t)\right| \leq \theta_{\alpha} \mathcal{M} f(x), \quad x \in \mathbb{R}^{n},
$$

where $\mathcal{M}$ denotes the Hardy-Littlewood maximal operator:

$$
\mathcal{M} f(x)=\sup _{r>0} r^{-n} \int_{B(x, r)}|f(y)| d y, x \in \mathbb{R}^{n} .
$$

(iv) There exists a constant $\eta_{n, \alpha}>0$ such that

$$
(x, t) \in T(O) \Rightarrow\left(p_{t}^{\alpha} *|f|\right)(x, t) \geq \eta_{n, \alpha},
$$

where $O$ is a bounded open set contained in $\operatorname{Int}\left(\left\{x \in \mathbb{R}^{n}: f(x) \geq 1\right\}\right)$.

Proof. (i) Since $\sup _{|y-x|<t}\left|P_{\alpha} f(x, t)\right|$ is lower semicontinuous on $\mathbb{R}^{n}$, we can see that $R_{s}^{\alpha, \beta}(f)$ is an open subset of $\mathbb{R}^{n}$ and

$$
\left\{\begin{array}{l}
L_{s}^{\alpha, \beta}(f) \subseteq T\left(R_{s}^{\alpha, \beta}(f)\right) \\
\mu\left(L_{s}^{\alpha, \beta}(f)\right) \leq \mu\left(T\left(R_{s}^{\alpha, \beta}(f)\right)\right)
\end{array}\right.
$$

Then

$$
\mu\left(L_{s}^{\alpha, \beta}(f) \cap T(B(0, k))\right) \leq \mu\left(T\left(R_{s}^{\alpha, \beta}(f) \cap T(B(0, k))\right)\right)=\mu\left(T\left(R_{s}^{\alpha, \beta}(f) \cap B(0, k)\right)\right) .
$$

(ii) It follows from the definition of $c_{p}^{\beta}(\mu ; t)$.

(iii) Since $p_{t}^{\alpha}(x)=\frac{1}{t^{n}} \psi\left(\frac{x}{t}\right)$, where $\psi(x):=\left(1+|x|^{2}\right)^{-(n+\alpha) / 2}$ is radial bounded and integrable on $\mathbb{R}^{n}$, it follows from [25, p.57, Proposition] that

$$
\sup _{|y-x|<t}\left|P_{\alpha} f(y, t)\right| \leq \theta_{\alpha} \mathcal{M} f(x) .
$$

(iv) For any $(x, t) \in T(O)$, we have

$$
B(x, t) \subseteq O \subseteq \operatorname{Int}(\{x: f(x)>1\}) .
$$

There exist $\sigma$ and $C$ which depend only on $n$ and $\alpha$ such that $\inf \left\{p_{t}^{\alpha}(x):|x|<\sigma t\right\} \geq C t^{-n}$. Then

$$
p_{t}^{\alpha} *|f|(x, t) \geq C t^{-n} \int_{B(x, \sigma t) \cap \operatorname{Int}(\{x: f(x) \geq 1\})}|f|(y) d y .
$$

If $\sigma>1$, then

$$
B(x, \sigma t) \cap \operatorname{Int}(\{x: f(x) \geq 1\}) \supseteq B(x, t) \cap \operatorname{Int}(\{x: f(x) \geq 1\})=B(x, t) .
$$

If $\sigma \leq 1$, then

$$
B(x, \sigma t) \cap \operatorname{Int}(\{x: f(x) \geq 1\})=B(x, \sigma t) .
$$

Thus $p_{t}^{\alpha} *|f|(x, t) \geq \eta_{n, \alpha}$ for a constant $\eta_{n, \alpha}>0$.

The following result provides the capacitary strong estimates for $C a p_{\mathbb{R}^{n}}^{\beta, p}(\cdot)$. For the proof, we refer the reader to [31, Lemma 2.1] and the references therein.

Lemma 2.13. Let $\beta \in(0, n)$ and $p \in[1, n / \beta]$. 
(i) For $f \in C_{0}^{\infty}\left(\mathbb{R}^{n}\right)$,

(ii) For $f \in C_{0}^{\infty}\left(\mathbb{R}^{n}\right)$,

$$
\int_{0}^{\infty} \operatorname{Cap}_{\mathbb{R}^{n}}^{\beta, p}\left(\left\{x \in \mathbb{R}^{n}:|f(x)| \geq s\right\}\right) d s^{p} \lesssim\|f\|_{\dot{W}^{\beta, p}\left(\mathbb{R}^{n}\right)}^{p}
$$

$$
\int_{0}^{\infty} \operatorname{Cap}_{\mathbb{R}^{n}}^{\beta, p}\left(\left\{x \in \mathbb{R}^{n}:|\mathcal{M} f(x)| \geq s\right\}\right) d s^{p} \lesssim\|f\|_{\dot{W}^{\beta, p}\left(\mathbb{R}^{n}\right)}^{p} .
$$

For handling the endpoint case $p=n / \beta$ when $p>q$, we need the following Riesz potentials on $\mathbb{R}^{2 n}$, see Adam-Xiao [5] and Adam [1] . For $\gamma \in(0,2 n)$,

$$
I_{\gamma}^{(2 n)} * f(z)=\int_{\mathbb{R}^{2 n}}|z-y|^{\gamma-2 n} f(y) d y, \quad z \in \mathbb{R}^{2 n} .
$$

For $\gamma \in(0,2 n), \dot{\mathcal{L}}_{\gamma}^{p}\left(\mathbb{R}^{2 n}\right)$ is defined as the completion of $I_{\gamma}^{2 n} * C_{0}^{\infty}\left(\mathbb{R}^{2 n}\right)$ with $\left\|I_{\gamma}^{2 n} * f\right\|_{\dot{\mathcal{L}}_{\gamma}^{p}\left(\mathbb{R}^{2 n)}\right.}=\|f\|_{L^{p}\left(\mathbb{R}^{2 n}\right)}$.

The following result is a special case of [1, Theorem 5.2] or [5, Theorem A].

Lemma 2.14. Let $\beta \in(0, n)$. Then there are a linear extension operator

$$
\mathcal{E}: \dot{W}^{\beta, n / \beta}\left(\mathbb{R}^{n}\right) \longrightarrow \dot{\mathcal{L}}_{2 \beta}^{n / \beta}\left(\mathbb{R}^{2 n}\right),
$$

and a linear restriction operator

$$
\mathcal{R}: \dot{\mathcal{L}}_{2 \beta}^{n / \beta}\left(\mathbb{R}^{2 n}\right) \longrightarrow \dot{W}^{\beta, n / \beta}\left(\mathbb{R}^{n}\right)
$$

such that $\mathcal{R E}$ is the identity. Moreover,

(i) For $f \in \dot{W}^{\beta, n / \beta}\left(\mathbb{R}^{n}\right),\|\mathcal{E} f\|_{\dot{\mathcal{L}}_{2 \beta}^{n \beta}\left(\mathbb{R}^{2 n}\right)} \lesssim\|f\|_{\dot{W}^{\beta, n / \beta}\left(\mathbb{R}^{n}\right)}$.

(ii) For $g \in \dot{\mathcal{L}}_{2 \beta}^{n / \beta}\left(\mathbb{R}^{2 n}\right),\|\mathcal{R} g\|_{\dot{W}^{\beta, n / \beta}\left(\mathbb{R}^{n}\right)} \lesssim\|g\|_{\dot{\mathcal{L}}_{2 \beta}^{n / \beta}\left(\mathbb{R}^{2 n}\right)}$.

Lemma 2.15. If $\alpha \in(0,2), \beta \in(0, n)$ and $(x, t) \in \mathbb{R}_{+}^{n+1}$, then

$$
\int_{\mathbb{R}^{n}} p_{t}^{\alpha}(y)|y-x|^{\beta-n} d y \lesssim\left(t^{2}+|x|^{2}\right)^{(\beta-n) / 2} .
$$

Proof. Define

$$
J(x, t)=\int_{\mathbb{R}^{n}} p_{t}^{\alpha}(y)|y-x|^{\beta-n} d y=c(n, \alpha) \int_{\mathbb{R}^{n}} \frac{t^{\alpha}|y-x|^{\beta-n}}{\left(|y|^{2}+t^{2}\right)^{(n+\alpha) / 2}} d y .
$$

Via the change of variables: $x \longrightarrow t x \& y \longrightarrow t y$, it is sufficient to show that

$$
J(x, 1) \lesssim\left(1+|x|^{2}\right)^{(\beta-n) / 2} .
$$

Since $J(0,1) \lesssim 1$, we may assume that $|x|>0$. Write $J(x, 1) \lesssim I_{1}(x)+I_{2}(x)$, where

$$
\left\{\begin{array}{l}
I_{1}(x):=\int_{B(x,|x| / 2)} \frac{|y-x|^{\beta-n}}{\left(|y|^{2}+1\right)^{(n+\alpha) / 2}} d y \\
I_{2}(x):=\int_{\mathbb{R}^{n} \backslash B(x,|x| / 2)} \frac{|y-x|^{\beta-n}}{\left(|y|^{2}+1\right)^{(n+\alpha) / 2}} d y .
\end{array}\right.
$$

Since $|x-y| \leq|x| / 2$ implies that $|y| \approx|x|$, we have

$$
\begin{aligned}
I_{1}(x) & \lesssim\left(1+|x|^{2}\right)^{-(n+\alpha) / 2} \int_{B(x,|x| / 2)}|y-x|^{\beta-n} d y \\
& \lesssim\left(1+|x|^{2}\right)^{(\beta-n) / 2} .
\end{aligned}
$$

If $|x-y|>|x| / 2$, then $|y-x|^{\beta-n} \lesssim|x|^{\beta-n}$, thus

$$
I_{2}(x) \lesssim|x|^{\beta-n} \int_{\mathbb{R}^{n} \backslash B(x,|x| / 2)} \frac{1}{\left(|y|^{2}+1\right)^{(n+\alpha) / 2}} d y \lesssim|x|^{\beta-n} .
$$


For $|x-y|>|x| / 2$, it holds $|y|<3|x-y|$ and

$$
I_{2}(x)=\int_{\mathbb{R}^{n} \backslash B(x,|x| / 2)} \frac{1}{\left(|y|^{2}+1\right)^{(n+\alpha) / 2}|y|^{n-\beta}} d y \lesssim 1 .
$$

So, $I_{2}(x) \lesssim\left(1+|x|^{2}\right)^{(\beta-n) / 2}$ and $J(x, 1) \lesssim\left(1+|x|^{2}\right)^{(\beta-n) / 2}$.

\section{EMbeddings of $L^{p}\left(\mathbb{R}^{n}\right)$ то $L^{q}\left(\mathbb{R}_{+}^{n+1}, \mu\right)$}

In this section, we focus on the embedding (1.4):

$$
\left\|P_{\alpha} f(\cdot, \cdot)\right\|_{L^{q}\left(\mathbb{R}_{+}^{n+1}, \mu\right)} \lesssim\|f\|_{L^{p}\left(\mathbb{R}^{n}\right)} .
$$

3.1. Embeddings of $L^{p}\left(\mathbb{R}^{n}\right)$ to $L^{q}\left(\mathbb{R}_{+}^{n+1}, \mu\right)$ when $p \leq q$. Given $1<p \leq q<\infty$. For $\lambda>0$, define

$$
c_{\alpha, p}(\mu ; \lambda):=\inf \left\{C_{\mathbb{R}_{+}^{n+1}}^{\alpha, p}(K): \text { compact } K \subset \mathbb{R}_{+}^{n+1}, \quad \mu(K) \geq \lambda\right\} .
$$

Theorem 3.1. Let $1<p \leq q<\infty$ and $\mu \in \mathcal{M}_{+}\left(\mathbb{R}_{+}^{n+1}\right)$.

(i) The embedding (1.4) holds if and only if

$$
\sup _{\lambda \in(0, \infty)} \lambda^{p / q} / c_{\alpha, p}(\mu ; \lambda)<\infty .
$$

(ii) If $1<p<q<\infty$, then $\sup _{\lambda \in(0, \infty)} \lambda^{p / q} / c_{\alpha, p}(\mu ; \lambda)<\infty$ can be replaced by

$$
\sup _{\left(r, x_{0}, t_{0}\right) \in(0, \infty) \times \mathbb{R}_{+}^{n+1}, t_{0} \leq r} \frac{\mu\left(B_{r}\left(x_{0}, t_{0}\right)\right)}{r^{q n / p}}<\infty .
$$

Proof. (i) Suppose that (1.4) is true. Let $K$ be a compact set in $\mathbb{R}_{+}^{n+1}$. Denote by $\left.\mu\right|_{K}$ the restriction of $\mu$ to the set $K$.

$$
\begin{aligned}
\left.\int_{\mathbb{R}^{n}} f(x) P_{\alpha}^{*} \mu\right|_{K}(x) d x & =\left.\int_{\mathbb{R}_{+}^{n+1}} P_{\alpha} f(x, t) d \mu\right|_{K}(x, t) \\
& \lesssim\left\|P_{\alpha} f\right\|_{L^{q}\left(\mathbb{R}_{+}^{n+1}, \mu\right)}(\mu(K))^{1 / q^{\prime}} \lesssim\|f\|_{L^{p}\left(\mathbb{R}^{n}\right)}(\mu(K))^{1 / q^{\prime}}
\end{aligned}
$$

which means that $\left\|\left.P_{\alpha}^{*} \mu\right|_{K}\right\|_{L^{p^{\prime}}\left(\mathbb{R}^{n}\right)} \lesssim(\mu(K))^{1 / q^{\prime}}$. Define

$$
E_{\lambda}(f)=\left\{(x, t) \in \mathbb{R}_{+}^{n+1}:\left|P_{\alpha} f(x, t)\right| \geq \lambda\right\} .
$$

We can get

$$
\begin{aligned}
\lambda \mu\left(E_{\lambda}(f)\right) & \leq\left.\int_{E_{\lambda}(f)}\left|P_{\alpha} f(x, t)\right| d \mu\right|_{E_{\lambda}(f)}(x, t) \\
& \leq\|f\|_{L^{p}\left(\mathbb{R}^{n}\right)}\left\|\left.P_{\alpha}^{*} \mu\right|_{E_{\lambda}(f)}\right\|_{L^{p^{\prime}}} \\
& \leq\|f\|_{L^{p}\left(\mathbb{R}^{n}\right)}\left(\mu\left(E_{\lambda}(f)\right)\right)^{1 / q^{\prime}} .
\end{aligned}
$$

This implies

$$
\sup _{\lambda \in(0, \infty)} \lambda^{q}\left(\mu\left(E_{\lambda}(f)\right)\right) \lesssim\|f\|_{L^{p}\left(\mathbb{R}^{n}\right)}^{q} .
$$

Choose a function $f \in L^{p}\left(\mathbb{R}^{n}\right)$ such that $P_{\alpha} f \geq 1$ on a given compact set $K \subset \mathbb{R}_{+}^{n+1}$, i.e., $K \subset E_{1}(f)$. We have

$$
(\mu(K))^{1 / q} \leq\left(\mu\left(E_{1}(f)\right)\right)^{1 / q} \lesssim\|f\|_{L^{p}\left(\mathbb{R}^{n}\right)} .
$$

Take the infimum on both sides of (3.1), we obtain $(\mu(K))^{1 / q} \lesssim\left(C_{\mathbb{R}_{+}^{n+1}}^{\alpha, p}(K)\right)^{1 / p}$. If $K$ is compact and $\mu(K) \geq \lambda$, then $\lambda^{p / q} \lesssim C_{\mathbb{R}_{+}^{n+1}}^{\alpha, p}(K)$ and hence, $\lambda^{p / q} \lesssim c(\mu ; \lambda)$ due to

$$
\lambda^{p / q} \lesssim \inf \left\{C_{\mathbb{R}_{+}^{n+1}}^{\alpha, p}(K), K \text { is compact and } \mu(K) \geq \lambda\right\} .
$$


Conversely, if $\sup _{\lambda \in(0, \infty)} \lambda^{p / q} / c_{\alpha, p}(\mu ; \lambda)<\infty$, then for any $\lambda>0, \lambda^{p / q} \lesssim C_{\mathbb{R}_{+}^{n+1}}^{\alpha, p}(K)$. For any $\tau$, according to Lemma2.11, one has

$$
\begin{aligned}
\tau^{p} C_{\mathbb{R}_{+}^{n+1}}^{\alpha, p}\left(E_{\tau}\right) & =\int_{0}^{\tau} C_{\mathbb{R}_{+}^{n+1}}^{\alpha, p}\left(\left\{(x, t) \in \mathbb{R}_{+}^{n+1}: P_{\alpha} f(x, t) \geq \tau\right\}\right) d \lambda^{p} \\
& \lesssim \int_{0}^{\tau} C_{\mathbb{R}_{+}^{n+1}}^{\alpha, p}\left(\left\{(x, t) \in \mathbb{R}_{+}^{n+1}: P_{\alpha} f(x, t) \geq \lambda\right\}\right) d \lambda^{p} \\
& \lesssim \int_{0}^{\infty} C_{\mathbb{R}_{+}^{n+1}}^{\alpha, p}\left(\left\{(x, t) \in \mathbb{R}_{+}^{n+1}: P_{\alpha} f(x, t) \geq \lambda\right\}\right) d \lambda^{p} \lesssim\|f\|_{L^{p}\left(\mathbb{R}^{n}\right)}^{p}
\end{aligned}
$$

By the layer cake representation, this implies

$$
\begin{aligned}
\int_{\mathbb{R}_{+}^{n+1}}\left|P_{\alpha} f(x, t)\right|^{q} d \mu(x, t) & =\int_{0}^{\infty} \mu\left(E_{\lambda}\right) d \lambda^{q} \\
& \lesssim \int_{0}^{\infty}\left(C_{\mathbb{R}_{+}^{n+1}}^{\alpha, p}\left(E_{\lambda}\right)\right)^{q / p} d \lambda^{q} \\
& \lesssim \int_{0}^{\infty}\left(\lambda^{-p}\|f\|_{L^{p}\left(\mathbb{R}^{n}\right)}^{p}\right)^{q / p-1}\left(C_{\mathbb{R}_{+}^{\alpha+1}}^{\alpha, p}\left(E_{\lambda}\right)\right) \lambda^{q-1} d \lambda \\
& \lesssim\|f\|_{L^{p}\left(\mathbb{R}^{n}\right)}^{q-p} \int_{0}^{\infty} C_{\mathbb{R}_{+}^{\alpha+1}}^{\alpha, p}\left(E_{\lambda}\right) d \lambda^{p} \\
& \lesssim\|f\|_{L^{p}\left(\mathbb{R}^{n}\right)}^{q}
\end{aligned}
$$

(ii) If $\lambda^{p / q} \lesssim c(\mu ; \lambda)$, then $\mu^{1 / q}(K) \lesssim\left(C_{\mathbb{R}_{+}^{n+1}}^{\alpha, p}(K)\right)^{1 / p}$ for any compact set $K \subset \mathbb{R}_{+}^{n+1}$. Let $K=B_{r}\left(x_{0}, t_{0}\right)$. By Theorem 2.10, for $t_{0} \lesssim r$,

$$
\left(\mu\left(B_{r}\left(x_{0}, t_{0}\right)\right)\right)^{1 / q} \lesssim\left(C_{\mathbb{R}_{+}^{\alpha+1}}^{\alpha, p}\left(B_{r}\left(x_{0}, t_{0}\right)\right)\right)^{1 / p} \lesssim r^{n / p}
$$

For the reverse, take a compact set $K$ such that $\mu(K) \geq \lambda$. If $(x, t) \in B_{r}\left(x_{0}, t_{0}\right),\left|x-x_{0}\right|<r / 2$ and $r+t_{0} \leq t \leq t_{0}+2 r$. If $\left|x-x_{0}\right|<r<t$, we can get $\left|x-x_{0}\right| / t<1$ and

$$
\begin{aligned}
p_{t}^{\alpha}\left(x-x_{0}\right) & =\frac{t^{\alpha}}{\left(t^{2}+\left|x-x_{0}\right|^{2}\right)^{(n+\alpha) / 2}} \\
& =\frac{1}{t^{n}} \frac{1}{\left(1+\left|x-x_{0}\right|^{2} / t^{2}\right)^{(n+\alpha) / 2}} \\
& \geq \frac{1}{2^{(n+\alpha) / 2}} \frac{1}{t^{n}} \\
& \gtrsim \frac{1}{3^{n} r^{n}} \frac{1}{2^{(n+\alpha) / 2}},
\end{aligned}
$$

equivalently, there exists a constant $c$ such that $r \geq c\left(p_{t}^{\alpha}\left(x-x_{0}\right)\right)^{-1 / n}$. Hence if $(x, t) \in B_{r}\left(t_{0}, x_{0}\right)$, then $r \in\left(c\left(p_{t}^{\alpha}\left(x-x_{0}\right)\right)^{-1 / n}, \infty\right)$. For this case, the characteristic functions

$$
1_{B_{r}\left(x_{0}, t_{0}\right)}(x, t)=1_{\left(c\left(p_{t}^{\alpha}\left(x-x_{0}\right)\right)^{-1 / n}, \infty\right)}(r)=1 .
$$


By Fubini's theorem, we can get

$$
\begin{aligned}
\left.\int_{0}^{\infty} \mu\right|_{K}\left(B_{r}\left(x_{0}, t_{0}\right)\right) \frac{d r}{r^{n+1}} & =\int_{0}^{\infty}\left\{\left.\int_{\mathbb{R}_{+}^{n+1}} 1_{B_{r}\left(x_{0}, t_{0}\right)}(x, t) d \mu\right|_{K}(x, t)\right\} \frac{d r}{r^{n+1}} \\
& =\left.\int_{\mathbb{R}_{+}^{n+1}}\left\{\int_{0}^{\infty} 1_{\left(c\left(p_{t}^{\alpha}\left(x-x_{0}\right)\right)^{-1 / n}, \infty\right)}(r) \frac{d r}{r^{n+1}}\right\} d \mu\right|_{K}(x, t) \\
& =\left.\int_{\mathbb{R}_{+}^{n+1}}\left\{\int_{\left(c\left(p_{t}^{\alpha}\left(x-x_{0}\right)\right)^{-1 / n}\right.}^{\infty} \frac{d r}{r^{n+1}}\right\} d \mu\right|_{K}(x, t) \\
& \left.\gtrsim \int_{\mathbb{R}_{+}^{n+1}} p_{t}^{\alpha}\left(x_{0}-x\right) d \mu\right|_{K}(x, t) \\
& =\left.P_{\alpha}^{*} \mu\right|_{K}\left(x_{0}\right) .
\end{aligned}
$$

By Minkowski’s inequality, we have

$$
\left\|\left.P_{\alpha}^{*} \mu\right|_{K}\right\|_{L^{p^{\prime}}\left(\mathbb{R}^{n}\right)} \lesssim \int_{0}^{\infty}\left\|\left.\mu\right|_{K}\left(B_{r_{0}}\left(\cdot, t_{0}\right)\right)\right\|_{L^{p^{\prime}}\left(\mathbb{R}^{n}\right)} \frac{d r}{r^{n+1}} .
$$

Take $\delta=(\mu(K))^{p / n q}$. On the one hand,

$$
\begin{aligned}
\left\|\left.\mu\right|_{K}\left(B_{r}\left(\cdot, t_{0}\right)\right)\right\|_{L^{p^{\prime}\left(\mathbb{R}^{n}\right)}}^{p^{\prime}} & =\int_{\mathbb{R}^{n}}\left(\mu\left(K \cap B_{r}\left(x_{0}, t_{0}\right)\right)\right)^{p^{\prime}} d x_{0} \\
& \lesssim(\mu(K))^{p^{\prime}-1} \int_{\mathbb{R}^{n}} \mu\left(K \cap B_{r}\left(x_{0}, t_{0}\right)\right) d x_{0} \\
& =(\mu(K))^{p^{\prime}-1} \int_{\mathbb{R}^{n}}\left(\int_{K \cap B_{r}\left(x_{0}, t_{0}\right)} 1 d \mu(t, y)\right) d x_{0} \\
& \lesssim(\mu(K))^{p^{\prime}-1} \int_{K}\left(\int_{\left|y-x_{0}\right|<r} d x_{0}\right) d \mu(t, y) \\
& \lesssim(\mu(K))^{p^{\prime}} r^{n}
\end{aligned}
$$

The above estimate gives

$$
\int_{\delta}^{\infty}\left\|\left.\mu\right|_{K}\left(B_{r}\left(\cdot, t_{0}\right)\right)\right\|_{L^{p^{\prime}\left(\mathbb{R}^{n}\right)}} \frac{d r}{r^{n+1}} \lesssim \int_{\delta}^{\infty} \mu(K) r^{n / p^{\prime}-n-1} d r \lesssim \mu(K) \delta^{-n / p} .
$$

Meanwhile, on the other hand, since $\mu\left(B_{r}\left(x_{0}, t_{0}\right)\right) \lesssim r^{n q / p}$, we can obtain

$$
\begin{aligned}
\left\|\left.\mu\right|_{K}\left(B_{r}\left(\cdot, t_{0}\right)\right)\right\|_{L^{p^{\prime}\left(\mathbb{R}^{n}\right)}}^{p^{\prime}} & \left.\lesssim r^{n q\left(p^{\prime}-1\right) / p} \int_{\mathbb{R}^{n}} \mu\right|_{K}\left(B_{r}\left(t_{0}, x_{0}\right)\right) d x_{0} \\
& =r^{n q\left(p^{\prime}-1\right) / p} \int_{\mathbb{R}^{n}}\left(\int_{K \cap B_{r}\left(x_{0}, t_{0}\right)} 1 d \mu(t, y)\right) d x_{0} \\
& \lesssim r^{n q\left(p^{\prime}-1\right) / p} \int_{K}\left(\int_{\left|y-x_{0}\right|<r} d x_{0}\right) d \mu(t, y) \\
& \lesssim \mu(K) r^{n+n q /\left(p^{2}-p\right)} .
\end{aligned}
$$

Hence, noting that $q>p$, we obtain

$$
\int_{0}^{\delta}\left\|\left.\mu\right|_{K}\left(B_{r}\left(\cdot, t_{0}\right)\right)\right\|_{L^{p^{\prime}}\left(\mathbb{R}^{n}\right)} \frac{d r}{r^{1+n}} \lesssim(\mu(K))^{1 / p^{\prime}} \delta^{n(q-p) / p^{2}} .
$$


Finally, we get

$$
\begin{aligned}
\left\|\left.P_{\alpha}^{*} \mu\right|_{K}\right\|_{L^{p^{\prime}\left(\mathbb{R}^{n}\right)}} & \lesssim \int_{0}^{\infty}\left\|\left.\mu\right|_{K}\left(B_{r}\left(\cdot, t_{0}\right)\right)\right\|_{L^{p^{\prime}\left(\mathbb{R}^{n}\right)}} \frac{d r}{r^{n+1}} \\
& =\left(\int_{0}^{\delta}+\int_{\delta}^{\infty}\right)\left\|\left.\mu\right|_{K}\left(B_{r}\left(\cdot, t_{0}\right)\right)\right\|_{L^{p^{\prime}\left(\mathbb{R}^{n}\right)}} \frac{d r}{r^{n+1}} \\
& \lesssim(\mu(K))^{1 / p^{\prime}} \delta^{n(q-p) / p^{2}}+(\mu(K)) \delta^{-n / p} \\
& \lesssim(\mu(K))^{1 / q^{\prime}} .
\end{aligned}
$$

Below we prove that $(\mu(K))^{1 / q} \leq\left(C_{\mathbb{R}_{+}^{n+1}}^{\alpha, p}(K)\right)^{1 / p}$ for any compact set $K \subset \mathbb{R}_{+}^{n+1}$. In fact, let $f \in C_{0}^{\infty}\left(\mathbb{R}^{n}\right)$ and $E_{\lambda, K}=\left\{(x, t) \in K:\left|P_{\alpha} * f(x, t)\right| \geq \lambda\right\}$. Then the set $E_{\lambda, K}$ is compact. It follows from (3.2) that

$$
\begin{aligned}
\lambda \mu\left(E_{\lambda, K}\right) & \leq\left.\int_{\mathbb{R}^{n}}\left|P_{\alpha} f(x, t)\right| d \mu\right|_{E_{\lambda, K}} \\
& \leq\|f\|_{L^{p}\left(\mathbb{R}^{n}\right)}\left\|\left.P_{\alpha}^{*} \mu\right|_{E_{\lambda, K}}\right\|_{L^{p^{\prime}}} \\
& \lesssim\|f\|_{L^{p}\left(\mathbb{R}^{n}\right)}\left(\mu\left(E_{\lambda, K}\right)\right)^{1 / q^{\prime}} .
\end{aligned}
$$

For an arbitrary $f \in L^{p}\left(\mathbb{R}^{n}\right)$, via approximating $f$ by a sequence from $C_{0}^{\infty}\left(\mathbb{R}^{n}\right)$ in the $L^{p}$-norm, we can prove that (3.3) holds for $f$. For $f \in L^{p}\left(\mathbb{R}^{n}\right)$ such that $P_{\alpha} f \geq 1$ on $K$, we have $E_{\lambda, K}=K$. Then (3.3) implies $(\mu(K))^{1 / q} \lesssim\|f\|_{L^{p}\left(\mathbb{R}^{n}\right)}$, which gives $(\mu(K))^{1 / q} \leq\left(C_{\mathbb{R}_{+}^{n+1}}^{\alpha, p}(K)\right)^{1 / p}$. Recall that $\mu(K) \geq \lambda$. Then taking the infimum over the compact sets $K$ such that $\mu(K) \geq \lambda$, we get $\lambda^{p / q} \lesssim C_{\mathbb{R}_{+}^{n+1}}^{\alpha, p}(K)$, i.e.,

$$
\lambda^{p / q} \lesssim \inf \left\{C_{\mathbb{R}_{+}^{n+1}}^{\alpha, p}(K): \quad \text { compact } K \subset \mathbb{R}_{+}^{n+1}, \quad \mu(K) \geq \lambda\right\}=c_{\alpha, p}(\mu ; \lambda) .
$$

3.2. Embeddings of $L^{p}\left(\mathbb{R}^{n}\right)$ to $L^{q}\left(\mathbb{R}_{+}^{n+1}, \mu\right)$ when $p>q$. Let $M \mu(x)=\sup _{r>0} r^{-n} \mu\left(B_{r}(x, r)\right)$ be the parabolic maximal function of a nonnegative Radon measure $\mu$ on $\mathbb{R}_{+}^{n+1}$. We show the embedding for $p>q$ inspired by some ideas from [24], which needs the following $L^{p}$-boundedness of $M \mu$.

Lemma 3.2. Let $1<p<\infty$. Then $\|M \mu\|_{L^{p}\left(\mathbb{R}^{n}\right)} \approx\left\|P_{\alpha}^{*} \mu\right\|_{L^{p}\left(\mathbb{R}^{n}\right)}$.

Proof. It is easy to check that for any $r>0$,

$$
P_{\alpha}^{*} \mu(x) \gtrsim \int_{B_{r}(x, r)} \frac{t^{\alpha}}{\left(t^{2}+|x-y|^{2}\right)^{(n+\alpha) / 2}} d \mu(y, t) \gtrsim r^{-n} \mu\left(B_{r}(x, r)\right),
$$

and so that $\|M \mu\|_{L^{p}\left(\mathbb{R}^{n}\right)} \lesssim\left\|P_{\alpha}^{*} \mu\right\|_{L^{p}\left(\mathbb{R}^{n}\right)}$. Next, we are about to prove the converse inequality by a slight modification of [4, Theorem 3.6.1]. Denote by $E_{\mu}(T, \lambda, r)=\left\{x \in \mathbb{R}^{n}: T \mu(x)>\lambda r\right\}$ for an operator $T$ and $(\lambda, r) \in(\mathbb{R}, \mathbb{R})$. Then by the famous good $\lambda$ inequality, there exist two constants $c_{1}>1$ and $c_{2}>0$ such that

$$
\left|E_{\mu}\left(P_{\alpha}^{*}, c_{1}, \rho\right)\right| \leq c_{2} \theta^{(n+\alpha) / n}\left|E_{\mu}\left(P_{\alpha}^{*}, 1, \rho\right)\right|+\left|E_{\mu}(M, \theta, \rho)\right| \text { for any } \rho>0 \text { and } 0<\theta \leq 1 .
$$

Hence, for any $\tau>0$, we get

$$
\int_{0}^{\tau}\left|E_{\mu}\left(P_{\alpha}^{*}, c_{1}, \rho\right)\right| \rho^{p-1} d \rho \leq c_{2} \theta^{(n+\alpha) / n} \int_{0}^{\tau}\left|E_{\mu}\left(P_{\alpha}^{*}, 1, \rho\right)\right| \rho^{p-1} d \rho+\int_{0}^{\tau}\left|E_{\mu}(M, \theta, \rho)\right| \rho^{p-1} d \rho .
$$

Namely,

$$
c_{1}^{-p} \int_{0}^{c_{1} \tau}\left|E_{\mu}\left(P_{\alpha}^{*}, 1, \rho\right)\right| \rho^{p-1} d \rho \leq c_{2} \theta^{(n+\alpha) / n} \int_{0}^{\tau}\left|E_{\mu}\left(P_{\alpha}^{*}, 1, \rho\right)\right| \rho^{p-1} d \rho+\theta^{-p} \int_{0}^{\theta \tau}\left|E_{\mu}(M, 1, \rho)\right| \rho^{p-1} d \rho .
$$

Accordingly,

$$
c_{1}^{-p} \int_{\mathbb{R}^{n}}\left(P_{\alpha}^{*} \mu(x)\right)^{p} d x \leq 2 \theta^{-p} \int_{\mathbb{R}^{n}}(M \mu(x))^{p} d x
$$


by letting $\theta$ be so small such that $c_{2} \theta^{(n+\alpha) / n} \leq \frac{1}{2} c_{1}^{-p}$ and $\tau \rightarrow \infty$. The foregoing analysis yields

$$
\|M \mu\|_{L^{p}\left(\mathbb{R}^{n}\right)} \gtrsim\left\|P_{\alpha}^{*} \mu\right\|_{L^{p}\left(\mathbb{R}^{n}\right)}
$$

which is the desired one.

Lemma 3.3. Denote by

$$
H_{p} \mu(x, t):=\int_{0}^{\infty}\left(r^{-n} \mu\left(B_{r}(x, t)\right)\right)^{p^{\prime}-1} r^{-1} d r
$$

the Hedberg-Wolff potential of $\mu$. Let $1<p<\infty$ and $\mu \in \mathcal{M}_{+}\left(\mathbb{R}_{+}^{n+1}\right)$. Then one has

$$
\left\|P_{\alpha}^{*} \mu\right\|_{L^{p^{\prime}}\left(\mathbb{R}^{n}\right)}^{p^{\prime}} \approx \int_{\mathbb{R}_{+}^{n+1}} H_{p} \mu d \mu
$$

Proof. We conclude from the fact

$$
\frac{\mu\left(B_{r}(x, r)\right)}{r^{n}} \lesssim\left(\int_{r}^{2 r}\left(\frac{\mu\left(B_{s}(x, s)\right)}{s^{n}}\right)^{p^{\prime}} \frac{d s}{s}\right)^{1 / p^{\prime}}
$$

that

$$
M \mu(x) \lesssim\left(\int_{0}^{\infty}\left(\frac{\mu\left(B_{s}(x, s)\right)}{s^{n}}\right)^{p^{\prime}} \frac{d s}{s}\right)^{1 / p^{\prime}}
$$

Since

by the Fubini theorem,

$$
\int_{\mathbb{R}^{n}} \int_{0}^{\infty}\left(\frac{\mu\left(B_{r}(x, r)\right)}{r^{n}}\right)^{p^{\prime}} \frac{d r d x}{r}=\int_{0}^{\infty} \int_{\mathbb{R}^{n}} \frac{\mu\left(B_{r}(x, r)\right)^{p^{\prime}}}{r^{n p^{\prime}+1}} d x d r
$$

$$
\int_{\mathbb{R}^{n}} \mu\left(B_{r}(x, r)\right)^{p^{\prime}} d x \lesssim \int_{B_{r}(y, r)} \int_{\mathbb{R}^{n}} \mu\left(B_{r}(x, r)\right)^{p^{\prime}-1} d x d \mu \lesssim r^{n} \int_{B_{\frac{3 r}{2}}\left(y, \frac{r}{2}\right)} \mu\left(B_{r}(y, r)\right)^{p^{\prime}-1} d \mu .
$$

Accordingly,

$$
\int_{0}^{\infty} \int_{\mathbb{R}^{n}} \frac{\mu\left(B_{r}(x, r)\right)^{p^{\prime}}}{r^{n p^{\prime}+1}} d x d r \lesssim \int_{\mathbb{R}_{+}^{1+n}}\left(\int_{0}^{\infty}\left(\frac{\mu\left(B_{r}(x, r)\right)}{r^{n}}\right)^{p^{\prime}-1} \frac{d r}{r}\right) d \mu
$$

which, together with Lemma 3.2, gives

$$
\left\|P_{\alpha}^{*} \mu\right\|_{L^{p^{\prime}\left(\mathbb{R}^{n}\right)}}^{p^{\prime}} \lesssim \int_{\mathbb{R}_{+}^{n+1}} H_{p} \mu d \mu
$$

Write

$$
B\left(y, 2^{-m}\right)=\left\{x \in \mathbb{R}^{n}:|x-y|<2^{-m} \& 2^{-m}<t<2^{1-m}\right\} \quad \forall m \in \mathbb{Z} \equiv\{0, \pm 1, \pm 2, \ldots\} .
$$

The converse inequality is a by-product of the following estimate

$$
\begin{aligned}
\int_{\mathbb{R}^{n}}\left(P_{\alpha}^{*} \mu(x)\right)^{p^{\prime}-1} p_{t}^{\alpha}(x-y) d x & \approx \int_{\mathbb{R}^{n}} \frac{t^{\alpha}}{\left(t^{2}+|x-y|^{2}\right)^{(n+\alpha) / 2}}\left(\int_{\mathbb{R}_{+}^{n+1}} \frac{s^{\alpha}}{\left(s^{2}+|x-z|^{2}\right)^{(n+\alpha) / 2}} d \mu\right)^{p^{\prime}-1} d x \\
& \gtrsim \sum_{m \in \mathbb{Z}} \int_{B\left(y, 2^{-m}\right)} t^{-n}\left(\int_{B_{2^{-m}(y, t)}} s^{-n} d \mu\right)^{p^{\prime}-1} d x \\
& \left.\gtrsim \sum_{m \in \mathbb{Z}} \int_{B\left(y, 2^{-m}\right)} 2^{m n}\left(\frac{\mu\left(B_{2}-m\right.}{2^{-m n}}\right)\right)^{p^{\prime}-1} d x \\
& \gtrsim \int_{0}^{\infty}\left(\frac{\mu\left(B_{r}(y, t)\right)}{r^{n}}\right)^{p^{\prime}-1} \frac{d r}{r}
\end{aligned}
$$


since

$$
\left\|P_{\alpha}^{*} \mu\right\|_{L^{p^{\prime}}\left(\mathbb{R}^{n}\right)}^{p^{\prime}}=\int_{\mathbb{R}^{n}}\left(P_{\alpha}^{*} \mu(x)\right)^{p^{\prime}-1}\left(P_{\alpha}^{*} \mu(x)\right) d x=\int_{\mathbb{R}_{+}^{n+1}} \int_{\mathbb{R}^{n}}\left(P_{\alpha}^{*} \mu(x)\right)^{p^{\prime}-1} p_{t}^{\alpha}(x-y) d x d \mu(y, t) .
$$

Therefore,

$$
\left\|P_{\alpha}^{*} \mu\right\|_{L^{p^{\prime}\left(\mathbb{R}^{n}\right)}}^{p^{\prime}} \gtrsim \int_{\mathbb{R}_{+}^{n+1}} H_{p} \mu d \mu
$$

as desired.

Now, we are ready to characterize the embedding (1.4) for $1<q<p<\infty$ as follows.

Theorem 3.4. Let $1<q<p<\infty$ and $\mu \in \mathcal{M}_{+}\left(\mathbb{R}_{+}^{n+1}\right)$. The following statements are true.

(i) The embedding (1.4) holds if and only if

$$
\int_{0}^{\infty}\left(\frac{\lambda^{p / q}}{c(\mu ; \lambda)}\right)^{q /(p-q)} \frac{d \lambda}{\lambda}<\infty
$$

(ii) The embedding (1.4) holds if and only if

$$
\int_{\mathbb{R}_{+}^{n+1}}\left\{\int_{0}^{\infty}\left(\frac{\mu\left(B_{r}\left(x_{0}, t_{0}\right)\right)}{C_{\mathbb{R}_{+}^{n+1}}^{\alpha, p}\left(B_{r}\left(x_{0}, t_{0}\right)\right)}\right)^{1 /(p-1)} \frac{d r}{r}\right\}^{q(p-1) /(p-q)} d \mu\left(x_{0}, t_{0}\right)<\infty .
$$

(iii) The embedding (1.4) holds if and only if $H_{p} \mu \in L^{q(p-1) /(p-q)}\left(\mathbb{R}_{+}^{n+1}, \mu\right)$.

Proof. It is easy to see that the statement (ii) is a consequence of the statement (iii). We only need to show statements (i) \& (iii). The rest of the proof is divided into two parts.

Part I: Proof of statement $(i)$. Let $P_{\alpha}: L^{p}\left(\mathbb{R}^{n}\right) \rightarrow L^{q}\left(\mathbb{R}_{+}^{n+1}, \mu\right)$ be bounded. Then

$$
\left(\int_{\mathbb{R}_{+}^{n+1}}\left|P_{\alpha} f(x, t)\right|^{q} d \mu\right)^{1 / q} \lesssim\|f\|_{L^{p}\left(\mathbb{R}^{n}\right)}
$$

By Markov's inequality, we can get

$$
\sup _{\lambda>0} \lambda\left(\mu\left(E_{\lambda}(f)\right)\right)^{1 / q} \lesssim\|f\|_{L^{p}\left(\mathbb{R}^{n}\right)},
$$

where $E_{\lambda}(f)=\left\{(x, t) \in \mathbb{R}_{+}^{n+1}:\left|P_{\alpha} f(x, t)\right| \geq \lambda\right\}$. By the layer cake representation, we get

$$
\left(\int_{0}^{\lambda} \mu\left(E_{\tau}(f)\right) \tau^{q-1} d \tau\right)^{1 / q} \lesssim\|f\|_{L^{p}\left(\mathbb{R}^{n}\right)} \quad \forall \lambda>0 .
$$

Then we have

$$
\left(\mu\left(E_{\lambda}(f)\right)\right)^{1 / q}\left(\int_{0}^{\lambda} \tau^{q-1} d \tau\right)^{1 / q} \lesssim\|f\|_{L^{p}\left(\mathbb{R}^{n}\right)} .
$$

For each integer $j$, there is a compact set $K_{j} \subset \mathbb{R}_{+}^{n+1}$ and a nonnegative function $f_{j} \in L^{p}\left(\mathbb{R}^{n}\right)$ such that

$$
\left\{\begin{array}{l}
C_{\mathbb{R}_{+}^{\alpha, p}}^{\alpha, p}\left(K_{j}\right) \leq 2 c_{\alpha, p}\left(\mu ; 2^{j}\right) ; \\
\mu\left(K_{j}\right)>2^{j} \\
P_{\alpha} f_{j} \geq 1_{K_{j}} ; \\
\left\|f_{j}\right\|_{L^{p}\left(\mathbb{R}^{n}\right)}^{p} \leq 2 C_{\mathbb{R}_{+}^{\alpha+1}}^{\alpha, p}\left(K_{j}\right) .
\end{array}\right.
$$


For the integers $i, k$ with $i<k$, let $f_{i, k}:=\sup _{i \leq j \leq k}\left(\frac{2^{j}}{c_{\alpha, p}\left(\mu ; 2^{j}\right)}\right)^{1 /(p-q)} f_{j}$. Then

$$
\begin{aligned}
\left\|f_{i, k}\right\|_{L^{p}\left(\mathbb{R}^{n}\right)}^{p} & \leq \sum_{j=i}^{k}\left(\frac{2^{j}}{c_{\alpha, p}\left(\mu ; 2^{j}\right)}\right)^{p /(p-q)}\left\|f_{j}\right\|_{L^{p}\left(\mathbb{R}^{n}\right)}^{p} \\
& \lesssim \sum_{j=i}^{k}\left(\frac{2^{j}}{c_{\alpha, p}\left(\mu ; 2^{j}\right)}\right)^{p /(p-q)} C_{\mathbb{R}_{+}^{\alpha, 1}}^{\alpha, p}\left(K_{j}\right) \\
& \lesssim \sum_{j=i}^{k}\left(\frac{2^{j}}{c_{\alpha, p}\left(\mu ; 2^{j}\right)}\right)^{p /(p-q)} c_{\alpha, p}\left(\mu ; 2^{j}\right) .
\end{aligned}
$$

Note that if $(x, t) \in K_{j}$, then

$$
\begin{aligned}
P_{\alpha} f_{i, k}(x, t) & =P_{\alpha}\left(\sup _{i \leq l \leq k}\left(\frac{2^{j}}{c_{\alpha, p}\left(\mu ; 2^{l}\right)}\right)^{1 /(p-q)} f_{l}(x, t)\right) \\
& \geq\left(\frac{2^{j}}{c_{\alpha, p}\left(\mu ; 2^{j}\right)}\right)^{1 /(p-q)} P_{\alpha} f_{j}(x, t) \\
& \geq\left(\frac{2^{j}}{c_{\alpha, p}\left(\mu ; 2^{j}\right)}\right)^{1 /(p-q)} .
\end{aligned}
$$

It can be seen from (3.5) that

$$
K_{j} \subset\left\{(x, t) \in \mathbb{R}_{+}^{n+1}: P_{\alpha} f_{i, k}(x, t) \geq\left(\frac{2^{j}}{c_{\alpha, p}\left(\mu ; 2^{j}\right)}\right)^{1 /(p-q)}\right\} .
$$

This means that

$$
2^{j}<\mu\left(K_{j}\right) \leq \mu\left(E_{\left(2^{j} / c_{\alpha, p}\left(\mu ; 2^{j}\right)\right)^{1 /(p-q)}}\left(f_{i, k}\right)\right) .
$$

In view of the embedding (1.4), we can use the (nonsymmetric) decreasing rearrangement ([7, Theorem $1.8]$ ), and then a simple computation to obtain

$$
\begin{aligned}
\left\|f_{i, k}\right\|_{L^{p}\left(\mathbb{R}^{n}\right)}^{q} & \approx \int_{\mathbb{R}_{+}^{n+1}}\left|P_{\alpha}\left(f_{i, k}\right)(x, t)\right|^{q} d \mu(x, t) \\
& \approx \int_{0}^{\infty}\left(\inf \left\{\lambda: \mu\left(E_{\lambda}\left(f_{i, k}\right)\right) \leq s\right\}\right)^{q} d s .
\end{aligned}
$$

Notice that 3.6 indicates that

$$
\inf \left\{\lambda: \mu\left(E_{\lambda}\left(f_{i, k}\right) \leq 2^{j}\right\}>\left(\frac{2^{j}}{c_{\alpha, p}\left(\mu ; 2^{j}\right)}\right)^{1 /(p-q)}\right.
$$

Then we split the integral to get

$$
\left\|f_{i, k}\right\|_{L^{p}\left(\mathbb{R}^{n}\right)}^{q} \geq \sum_{j=i}^{k} 2^{j}\left(\inf \left\{\lambda: \mu\left(E_{\lambda}\left(f_{i, k}\right)\right) \leq 2^{j}\right\}\right)^{q} \geq \sum_{j=i}^{k} 2^{j}\left(\frac{2^{j}}{c_{\alpha, p}\left(\mu ; 2^{j}\right)}\right)^{q /(p-q)} .
$$


Finally, applying (3.4) and a direct computation, we have

$$
\begin{aligned}
\left\|f_{i, k}\right\|_{L^{p}\left(\mathbb{R}^{n}\right)}^{q} & \geq\left(\frac{\sum_{j=i}^{k}\left(2^{j} / c_{\alpha, p}\left(\mu ; 2^{j}\right)\right)^{q /(p-q)} 2^{j}}{\left(\sum_{j=i}^{k}\left(2^{j} / c_{\alpha, p}\left(\mu ; 2^{j}\right)\right)^{p /(p-q)} c_{\alpha, p}\left(\mu ; 2^{j}\right)\right)^{q / p}}\right)\left\|f_{i, k}\right\|_{L^{p}\left(\mathbb{R}^{n}\right)}^{q} \\
& \approx\left(\sum_{j=i}^{k} \frac{2^{j p /(p-q)}}{\left(c_{\alpha, p}\left(\mu ; 2^{j}\right)\right)^{q /(p-q)}}\right)^{(p-q) / p}\left\|f_{i, k}\right\|_{L^{p}\left(\mathbb{R}^{n}\right)}^{q},
\end{aligned}
$$

which implies

$$
\sum_{j=i}^{k} \frac{2^{j p /(p-q)}}{\left(c_{\alpha, p}\left(\mu ; 2^{j}\right)\right)^{q /(p-q)}} \lesssim 1
$$

and hence

$$
\int_{0}^{\infty}\left(\lambda^{p / q} / c_{\alpha, p}(\mu ; \lambda)\right)^{q /(p-q)} \lambda^{-1} d \lambda \lesssim \sum_{j=-\infty}^{\infty} \frac{2^{j p /(p-q)}}{\left(c_{\alpha, p}\left(\mu ; 2^{j}\right)\right)^{q /(p-q)}} \lesssim 1 .
$$

Conversely, let

$$
I_{p, q}(\mu)=\int_{0}^{\infty}\left(\frac{\lambda^{p / q}}{c_{\alpha, p}(\mu ; \lambda)}\right)^{q /(p-q)} \frac{d \lambda}{\lambda}<\infty
$$

Now for $f \in C_{0}^{\infty}\left(\mathbb{R}^{n}\right)$, let

$$
S_{p, q}(\mu ; f)=\sum_{j=-\infty}^{\infty} \frac{\left(\mu\left(E_{2^{j}}(f)\right)-\mu\left(E_{2^{j+1}}(f)\right)\right)^{p /(p-q)}}{\left(C_{\mathbb{R}_{+}^{n+1}}^{\alpha, p}\left(E_{2^{j}}(f)\right)\right)^{q /(p-q)}}
$$

Using layer-cake representation, Hölder's inequality and Lemma2.11, we obtain

$$
\begin{aligned}
& \int_{\mathbb{R}_{+}^{n+1}}\left|P_{\alpha} f(x, t)\right|^{q} d \mu(x, t) \\
& =q \int_{0}^{\infty} \lambda^{q-1} \mu\left(E_{\lambda}(f)\right) d \lambda \\
& \lesssim \sum_{j=-\infty}^{\infty}\left(\mu\left(E_{2^{j}}(f)\right)-\mu\left(E_{2^{j+1}}(f)\right) 2^{j q}\right. \\
& \lesssim\left(S_{p, q}(\mu ; f)\right)^{(p-q) / p}\left(\sum_{j=-\infty}^{\infty} 2^{j p} C_{\mathbb{R}_{+}^{\alpha, p}}^{\alpha, p}\left(E_{2^{j}}(f)\right)\right)^{q / p} \\
& \lesssim\left(S_{p, q}(\mu ; f)\right)^{(p-q) / p}\left(\int_{0}^{\infty} C_{\mathbb{R}_{+}^{\alpha+1}}^{\alpha, p}\left(\left\{(x, t) \in \mathbb{R}_{+}^{n+1}:\left|P_{\alpha} f(x, t)\right|>\lambda\right\}\right) d \lambda^{p}\right)^{q / p} \\
& \lesssim\left(S_{p, q}(\mu ; f)\right)^{(p-q) / p}\|f\|_{L^{p}\left(\mathbb{R}^{n}\right)}^{q}
\end{aligned}
$$


Note also that

$$
\begin{aligned}
\left(S_{p, q}(\mu ; f)\right)^{(p-q) / p} & =\left\{\sum_{j=-\infty}^{\infty} \frac{\left(\mu\left(E_{2^{j}}(f)\right)-\mu\left(E_{2^{j+1}}(f)\right)\right)^{p /(p-q)}}{\left(C_{\mathbb{R}_{+}^{\alpha+1}}^{\alpha, p}\left(E_{2^{j}}(f)\right)\right)^{q /(p-q)}}\right\}^{(p-q) / p} \\
& \lesssim\left\{\sum_{j=-\infty}^{\infty} \frac{\left(\mu\left(E_{2^{j}}(f)\right)-\mu\left(E_{2^{j+1}}(f)\right)\right)^{p /(p-q)}}{\left(c_{\alpha, p}\left(\mu ; \mu\left(E_{2^{j}}(f)\right)\right)\right)^{q /(p-q)}}\right\}^{(p-q) / p} \\
& \lesssim\left\{\sum_{j=-\infty}^{\infty} \frac{\left(\mu\left(E_{2^{j}}(f)\right)\right)^{p /(p-q)}-\left(\mu\left(E_{2^{j+1}}(f)\right)\right)^{p /(p-q)}}{\left(c_{\alpha, p}\left(\mu ; \mu\left(E_{2^{j}}(f)\right)\right)\right)^{q /(p-q)}}\right\}^{(p-q) / p} \\
& \lesssim\left(\int_{0}^{\infty} \frac{d s^{p /(p-q)}}{\left(c_{\alpha, p}(\mu ; s)\right)^{q /(p-q)}}\right)^{(p-q) / p} \\
& \simeq\left(I_{p, q}(\mu)\right)^{(p-q) / p} .
\end{aligned}
$$

Therefore,

$$
\left(\int_{\mathbb{R}_{+}^{n+1}}\left|P_{\alpha} f(x, t)\right|^{q} d \mu(x, t)\right)^{1 / q} \lesssim\left(I_{p, q}(\mu)\right)^{(p-q) / p q}\|f\|_{L^{p}\left(\mathbb{R}^{n}\right)} .
$$

Part II: Proof of statements (iii). Similar to [12, Theorem 2.1], this part consists of two steps.

Step 1 - proving that $(1.4) \Rightarrow H_{p} \mu \in L_{\mu}^{q(p-1) /(p-q)}\left(\mathbb{R}_{+}^{n+1}\right)$.

For $m_{0} \in \mathbb{Z}_{+}=0,1,2, \ldots$, and $m_{k} \in \mathbb{Z}, k=1,2, \ldots$, denote by $\Theta_{l}$ the following dyadic cube with side length $l \equiv l\left(\Theta_{l}\right)$ :

$$
\Theta_{l} \equiv\left(m_{1} l,\left(m_{1}+1\right) l\right) \times \cdots \times\left(m_{n} l,\left(m_{n}+1\right) l\right) \times\left(m_{0} l,\left(m_{0}+1\right) l\right) .
$$

Let $\boldsymbol{\Theta}=\left\{\Theta_{l}\right\}$ be the family of all the above-defined-dyadic cubes in $\mathbb{R}_{+}^{n+1}$. Setting

$$
H_{p}^{d} \mu:=H_{p}^{d} \mu(x, t)=\sum_{\Theta_{l} \in \boldsymbol{\Theta}}\left(\mu\left(\Theta_{l}\right) / l^{n}\right)^{p^{\prime}-1} \mathbf{1}_{\Theta_{l}}(x, t),
$$

we first show that

$$
\text { (1.4) } \Rightarrow \int_{\mathbb{R}_{+}^{n+1}}\left(H_{p}^{d} \mu\right)^{q(p-1) /(p-q)} d \mu<\infty \text {. }
$$

In fact, (1.4) is equivalent to the following inequality by the duality

$$
\left\|P_{\alpha}^{*}(f d \mu)\right\|_{L^{p^{\prime}\left(\mathbb{R}^{n}\right)}}^{p^{\prime}} \lesssim\|f\|_{L^{q^{\prime}\left(\mathbb{R}_{+}^{n+1}, \mu\right)}}^{p^{\prime}} \quad \forall f \in L^{q^{\prime}}\left(\mathbb{R}_{+}^{n+1}, \mu\right) .
$$

Applying Lemma 3.3 to $H_{p}^{d} \mu$ and $f d \mu$, and using Jensen's inequality, we thus get

$$
\left\|P_{\alpha}^{*}(f d \mu)\right\|_{L^{p^{\prime}\left(\mathbb{R}^{n}\right)}}^{p^{\prime}} \gtrsim \int_{\mathbb{R}_{+}^{n+1}} H_{p}^{d}(f d \mu)(x, t) f(x, t) d \mu \gtrsim \sum_{\Theta_{l}}\left\{\int_{\Theta_{l}} f(x, t) d \mu\right\}^{p^{\prime}} l^{n\left(1-p^{\prime}\right)},
$$

and hence

$$
\sum_{\Theta_{l}}\left\{\int_{\Theta_{l}} f(x, t) d \mu\right\}^{p^{\prime}} l^{n\left(1-p^{\prime}\right)} \lesssim\|f\|_{L_{\mu}^{q^{\prime}\left(\mathbb{R}_{+}^{n+1}\right)}}^{p^{\prime}}
$$

which is equivalent to

$$
\sum_{\Theta_{l}} g_{\Theta_{l}}\left(\int_{\Theta_{l}} f(x, t) d \mu(x, t)\right)^{p^{\prime}}\left(\mu\left(\Theta_{l}\right)\right)^{-p^{\prime}} \lesssim\|f\|_{\left.L_{\mu}^{q^{\prime}} \mathbb{R}_{+}^{n+1}\right)}^{p^{\prime}} \quad \text { with } \quad g_{\Theta_{l}}=\left(\mu\left(\Theta_{l}\right)\right)^{p^{\prime}} l^{n\left(1-p^{\prime}\right)} .
$$


For $0 \leq \widetilde{f} \in L_{\mu}^{q^{\prime} / p^{\prime}}\left(\mathbb{R}_{+}^{n+1}\right)$, set $M(x, t)=\left(M_{\mu}^{d} \widetilde{f}\right)^{1 / p^{\prime}}(x, t)$. Here $M_{\mu}^{d}$ is the dyadic Hardy-Littlewood maximal function defined as

$$
M_{\mu}^{d} f(x, t)=\sup _{(x, t) \in \Theta_{l} \in \Theta} \frac{1}{\mu\left(\Theta_{l}\right)} \int_{\Theta_{l}}|f(y, s)| d \mu .
$$

Applying (3.8) to $M$ in place of $f$, and using $\|M\|_{L^{q^{\prime}}\left(\mathbb{R}_{+}^{n+1}, \mu\right)}^{p^{\prime}} \lesssim\|\widetilde{f}\|_{L^{q^{\prime}} / p^{\prime}\left(\mathbb{R}_{+}^{n+1}, \mu\right)}$, we find

$$
\sum_{\Theta_{l}} g_{\Theta_{l}}\left(\frac{1}{\mu\left(\Theta_{l}\right)} \int_{\Theta_{l}} f(x, t) d \mu(x, y)\right)^{p^{\prime}} \lesssim\|M\|_{L^{q^{\prime}}\left(\mathbb{R}_{+}^{n+1}, \mu\right)}^{p^{\prime}} \lesssim\|\widetilde{f}\|_{L^{q^{\prime} / p^{\prime}}\left(\mathbb{R}_{+}^{n+1}, \mu\right)} .
$$

Then using

$$
\left(\frac{1}{\mu\left(\Theta_{l}\right)} \int_{\Theta_{l}} M(x, t) d \mu\right)^{p^{\prime}} \gtrsim \frac{1}{\mu\left(\Theta_{l}\right)} \int_{\Theta_{l}} \widetilde{f}(x, t) d \mu,
$$

one has

$$
\sum_{\Theta_{l}} \frac{g_{\Theta_{l}}}{\mu\left(\Theta_{l}\right)} \int_{\Theta_{l}} \widetilde{f}(x, t) d \mu \lesssim\|\widetilde{f}\|_{L^{q^{\prime} / p^{\prime}\left(\mathbb{R}_{+}^{n+1}, \mu\right)}} .
$$

Thereby getting by the duality that

$$
\int_{\mathbb{R}_{+}^{n+1}} \sum_{\Theta_{l}} \frac{g_{\Theta_{l}}}{\mu\left(\Theta_{l}\right)} \mathbf{1}_{\Theta_{l}} \tilde{f}(x, t) d \mu=\sum_{\Theta_{l}} \frac{g_{\Theta_{l}}}{\mu\left(\Theta_{l}\right)} \mathbf{1}_{\Theta_{l}} \in L^{q^{\prime} /\left(q^{\prime}-p^{\prime}\right)}\left(\mathbb{R}_{+}^{n+1}, \mu\right)
$$

i.e.

$$
\sum_{\Theta_{l}}\left(\frac{\mu\left(\Theta_{l}\right)}{l^{n}}\right)^{p^{\prime}-1} \mathbf{1}_{\Theta_{l}} \in L^{q(p-1) /(p-q)}\left(\mathbb{R}_{+}^{n+1}, \mu\right),
$$

which shows (3.7) as desired.

We proceed the proof by letting

$$
\left\{\begin{array}{l}
H_{p}^{d, \lambda} \mu(x, t)=\sum_{\Theta_{l} \in \Theta_{\lambda}}\left(\frac{\mu\left(\Theta_{l}\right)}{l^{l}}\right)^{p^{\prime}-1} \mathbf{1}_{\Theta_{l}}(x, t) \\
\boldsymbol{\Theta}_{\lambda}=\boldsymbol{\Theta}+\lambda=\left\{\Theta_{l}+\lambda\right\}_{\Theta_{l} \in \Theta} ; \\
\Theta_{l}+\lambda=\left\{(x, t)+\lambda:(x, t) \in \Theta_{l}\right\} .
\end{array}\right.
$$

Then

$$
\sup _{\lambda \in \mathbb{R}_{+}^{n+1}} \int_{\mathbb{R}_{+}^{n+1}}\left(H_{p}^{d, \lambda} \mu(x, t)\right)^{q(p-1) /(p-q)} d \mu<\infty
$$

by the arguments leading to 3.7 (apply just the same for $\Theta_{l}$ instead of $\Theta$ ). Hence Step 1 is completed by showing that

$$
H_{p} \mu \in L_{\mu}^{q(p-1) /(p-q)}\left(\mathbb{R}_{+}^{n+1}\right),
$$

which will be considered in two cases.

Case 1. $\mu$ is a doubling measure. (3.10) can be obtained by (3.7) and the following estimate

$$
H_{p} \mu(x, t) \lesssim \sum_{\Theta_{l}}\left(\frac{\mu\left(\Theta_{l}^{*}\right)}{l^{n}}\right)^{p^{\prime}-1} \mathbf{1}_{\Theta_{l}}(x, t)
$$

where $\Theta_{l}^{*}$ is the cube with the same center as $\Theta_{l}$ and side length two times as $\Theta_{l}$.

Case 2. $\mu$ is a possibly non-doubling measure. In this case, we first claim that for any $\delta>0$,

$$
H_{p, \delta} \mu(x, t):=\int_{0}^{\delta}\left(\frac{\mu\left(B_{r}(x, t)\right)}{r^{n}}\right)^{p^{\prime}-1} \frac{d r}{r} \lesssim \delta^{-(n+1)} \int_{|\lambda| \lesssim \delta} H_{p}^{d, \lambda} \mu(x, t) d \lambda .
$$


Indeed, for fixed $x \in \mathbb{R}^{n}$ and $\delta>0$ with $2^{i-1} \xi \leq \delta<2^{i} \xi$, where $i \in \mathbb{Z}$ and $\xi>0$ will be determined later, we have

$$
H_{p, \delta} \mu(x, t) \lesssim \sum_{j=-\infty}^{i}\left(\frac{\mu\left(B_{2^{j} \xi}(x, t)\right)}{\left(2^{j} \xi\right)^{n}}\right)^{p^{\prime}-1}
$$

Assume that $\Theta_{h, j}$ is a cube centered at $x$, of length $h$, with $2^{j-1}<h \leq 2^{j}$ for $j \leq i$, then $B_{2^{j} \xi}(x, t) \subseteq \Theta_{h, j}$ for sufficiently small $\xi$ and $\xi$ does not depend on $x$. Suppose that $F=\left\{\lambda: \lambda \in \mathbb{R}_{+}^{n+1},|\lambda| \lesssim \delta\right\}$ and there exists $\Theta_{l}^{\lambda} \in \Theta_{l}$ satisfying $l=2^{j+1}$ and $\Theta_{l, j} \subseteq \Theta_{l}^{\lambda}$. Then it is immediate that there is a constant $c(n)>0$ such that $|F| \geq c(n) \delta^{n+1}$ by a geometric consideration. Therefore, one has

$$
\begin{aligned}
\mu\left(B_{2^{j} \xi}(x, t)\right)^{p^{\prime}-1} & \lesssim|F|^{-1} \int_{F} \sum_{\Theta_{l}^{\lambda} \in \Theta^{\lambda}} \mu\left(\Theta_{l}^{\lambda}\right)^{p^{\prime}-1} \mathbf{1}_{\Theta_{l}^{\lambda}}(x, t) d \lambda \\
& \lesssim \delta^{-(n+1)} \int_{|\lambda| \lesssim \delta} \sum_{\Theta_{l}^{\lambda} \in \Theta^{\lambda}} \mu\left(\Theta_{l}^{\lambda}\right)^{p^{\prime}-1} \mathbf{1}_{\Theta_{l}^{\lambda}}(x, t) d \lambda,
\end{aligned}
$$

with $l=2^{j+1}$ and $B_{2^{j} \xi}(x, t) \subset \Theta_{l}^{\lambda}$, which clearly implies

$$
H_{p, \delta} \mu(x, t) \lesssim \delta^{-(n+1)} \int_{|\lambda| \lesssim \delta} \sum_{j=-\infty}^{i} \sum_{l=2^{j+1}}\left(\frac{\mu\left(\Theta_{l}^{\lambda}\right)}{\left(2^{j} \xi\right)^{n}}\right)^{p^{\prime}-1} \mathbf{1}_{\Theta_{l}^{\lambda}}(x, t) d s \lesssim \delta^{-(n+1)} \int_{|\lambda| \lesssim \delta} H_{p}^{d, \lambda} \mu(x, t) d \lambda
$$

as the desired inequality (3.11). This, along with Hölder's inequality and the Fubini theorem, implies

$$
\begin{aligned}
& \int_{\mathbb{R}_{+}^{n+1}}\left(H_{p, \delta} \mu(x, t)\right)^{q(p-1) /(p-q)} d \mu(x, t) \\
& \lesssim \int_{\mathbb{R}_{+}^{n+1}}\left\{\frac{1}{\delta^{(n+1)}}\left(\int_{|\lambda| \lesssim C \delta}\left(H_{p}^{d, \lambda} \mu(x, t)\right)^{q(p-1) /(p-q)} d \lambda\right)^{(p-q) /[q(p-1)]}\left(\int_{|\lambda| \lesssim \delta} d \lambda\right)^{p(q-1) /[q(p-1)]}\right\}^{q(p-1) /(p-q)} d \mu \\
& \lesssim \delta^{-(n+1)} \int_{|\lambda| \lesssim \delta}\left(\int_{\mathbb{R}_{+}^{n+1}}\left(H_{p}^{d, \lambda} \mu(x, t)\right)^{q(p-1) /(p-q)} d \mu\right) d \lambda \\
& \leq C(n) .
\end{aligned}
$$

In the last step, we used (3.9). The constant $C(n)$ is independent of $\delta$. (3.10) follows readily from the monotone convergence theorem and the above inequality by letting $\delta \rightarrow \infty$.

Step 2 - showing that $H_{p} \mu \in L_{\mu}^{q(p-1) /(p-q)}\left(\mathbb{R}_{+}^{n+1}\right) \Rightarrow(1.4)$.

Utilizing Lemma 3.3, it is sufficient to show that

$$
H_{p} \mu \in L_{\mu}^{q(p-1) /(p-q)}\left(\mathbb{R}_{+}^{n+1}\right) \Rightarrow \int_{\mathbb{R}_{+}^{n+1}} H_{p}(f d \mu)(x, t) f(x, t) d \mu(x, t) \lesssim\|f\|_{L_{\mu}^{q^{\prime}}\left(\mathbb{R}_{+}^{n+1}\right)}^{p^{\prime}} \quad \forall f \in L_{\mu}^{q^{\prime}}\left(\mathbb{R}_{+}^{n+1}\right),
$$

since (1.4) is equivalent to

$$
\left\|P_{\alpha}^{*}(f d \mu)\right\|_{L^{p^{\prime}\left(\mathbb{R}^{n}\right)}} \lesssim\|f\|_{L_{\mu}^{q^{\prime}\left(\mathbb{R}_{+}^{n+1}\right)}} .
$$

Without loss of generality, we assume $f \geq 0$ in the following analysis. Let

$$
M_{\mu} f(x, t)=\sup _{r>0} \frac{1}{\mu\left(B_{r}(x, t)\right)} \int_{B_{r}(x, t)} f(y, s) d \mu(y, s)
$$


denote the centered Hardy-Littlewood maximal function of $f$ with respect to $\mu$. Then the Hölder inequality shows

$$
\begin{aligned}
& \int_{\mathbb{R}_{+}^{n+1}} H_{p}(f d \mu)(x, t) f(x, t) d \mu(x, t) \\
& \lesssim \int_{\mathbb{R}_{+}^{n+1}}\left(M_{\mu} f(x, t)\right)^{p^{\prime}-1} H_{p} \mu(x, t) f(x, t) d \mu(x, t) \\
& \lesssim\left(\int_{\mathbb{R}_{+}^{n+1}}\left(M_{\mu} f(x, t)\right)^{q^{\prime}} d \mu(x, t)\right)^{\left(p^{\prime}-1\right) / q^{\prime}}\left(\int_{\mathbb{R}_{+}^{n+1}}\left(f(x, t) H_{p} \mu(x, t)\right)^{q^{\prime} /\left(q^{\prime}-p^{\prime}+1\right)} d \mu(x, t)\right)^{\left(q^{\prime}-p^{\prime}+1\right) / q^{\prime}}
\end{aligned}
$$

via the following observation

$$
\begin{aligned}
H_{p}(f d \mu)(x, t) & \approx \int_{0}^{\infty}\left(\frac{\mu\left(B_{r}(x, t)\right)}{r^{n}}\right)^{p^{\prime}-1}\left(\frac{1}{\mu\left(B_{r}(x, t)\right)} \int_{B_{r}(x, t)} f(x, t) d \mu(x, t)\right)^{p^{\prime}-1} \frac{d r}{r} \\
& \lesssim\left(M_{\mu} f(x, t)\right)^{p^{\prime}-1} H_{p} \mu(x, t) .
\end{aligned}
$$

We conclude from the $L_{\mu}^{q^{\prime}}\left(\mathbb{R}_{+}^{n+1}\right)$-boundedness of $M_{\mu}$ (cf.[15]) and a further use of the Hölder inequality that

$$
\int_{\mathbb{R}_{+}^{n+1}} H_{p}(f d \mu)(x, t) f(x, t) d \mu(x, t) \lesssim\|f\|_{L_{\mu}^{q^{\prime}\left(\mathbb{R}_{+}^{n+1}\right)}}^{p^{\prime}}\left(\int_{\mathbb{R}_{+}^{n+1}}\left(H_{p} \mu\right)^{q(p-1) /(p-q)} d \mu(x, t)\right)^{(p-q) /[q(p-1)]}
$$

as desired.

$$
\text { 4. EMbeddings of } \dot{W}^{\beta, p}\left(\mathbb{R}^{n}\right) \text { то } L^{q}\left(\mathbb{R}_{+}^{n+1}, \mu\right)
$$

In this section, we will characterize the embedding (1.5)

$$
\left\|P_{\alpha} f(\cdot, \cdot)\right\|_{L^{q}\left(\mathbb{R}_{+}^{n+1}, \mu\right)} \lesssim\|f\|_{\dot{W}^{\beta, p}\left(\mathbb{R}^{n}\right)}
$$

in terms of the capacity and the fractional perimeter of open balls.

\subsection{Embeddings of $\dot{W}^{\beta, p}\left(\mathbb{R}^{n}\right)$ to $L^{q}\left(\mathbb{R}_{+}^{n+1}, \mu\right)$ when $1 \leq p \leq \min \{n / \beta, q\}$.}

Proposition 4.1. Let $\beta \in(0, n), 1 \leq p \leq \min \{n / \beta, q\}$, and $\mu \in \mathcal{M}_{+}\left(\mathbb{R}_{+}^{n+1}\right)$. Then the following statements are equivalent:

(i) $\left\|P_{\alpha} f(\cdot, \cdot)\right\|_{L^{q, p}\left(\mathbb{R}_{+}^{n+1}, \mu\right)} \lesssim\|f\|_{\dot{W}^{\beta, p}\left(\mathbb{R}^{n}\right)} \quad \forall f \in C_{0}^{\infty}\left(\mathbb{R}^{n}\right)$;

(ii) $\left\|P_{\alpha} f(\cdot, \cdot)\right\|_{L^{q}\left(\mathbb{R}_{+}^{n+1}, \mu\right)} \lesssim\|f\|_{\dot{W}^{\beta, p}\left(\mathbb{R}^{n}\right)} \quad \forall f \in C_{0}^{\infty}\left(\mathbb{R}^{n}\right)$;

(iii) $\left\|P_{\alpha} f(\cdot, \cdot)\right\|_{L^{q, \infty}\left(\mathbb{R}_{+}^{n+1}, \mu\right)} \lesssim\|f\|_{\dot{W}^{\beta, p}\left(\mathbb{R}^{n}\right)} \quad \forall f \in C_{0}^{\infty}\left(\mathbb{R}^{n}\right)$;

(iv) $\sup _{t>0} \frac{t^{p / q}}{c_{p}^{\beta}(\mu ; t)}<\infty$;

(v) $(\mu(T(O)))^{p / q} \lesssim C a p_{\mathbb{R}^{n}}^{\beta, p}(O)$ holds for any bounded open set $O \subseteq \mathbb{R}^{n}$.

Proof. The implications (i) $\Longrightarrow$ (ii) $\Longrightarrow$ (iii) can be deduced from

$$
\left(s^{q} \mu\left(L_{s}^{\alpha, \beta}(f)\right)\right)^{p / q} \leq\left(q \int_{0}^{\infty} \mu\left(L_{s}^{\alpha, \beta}(f)\right) s^{q-1} d s\right)^{p / q} \leq \int_{0}^{\infty}\left(\mu\left(L_{s}^{\alpha, \beta}(f)\right)\right)^{p / q} d s^{p}
$$

since

$$
q \mu\left(L_{s}^{\alpha, \beta}(f)\right) s^{q-1} \leq \frac{d}{d s}\left(\int_{0}^{s}\left(\mu\left(L_{t}^{\alpha, \beta}(f)\right)\right)^{p / q} d t^{p}\right)^{q / p} .
$$

Now, we prove (iii) $\Longrightarrow(\mathrm{v}) \Longrightarrow$ (i). If (iii) is true,

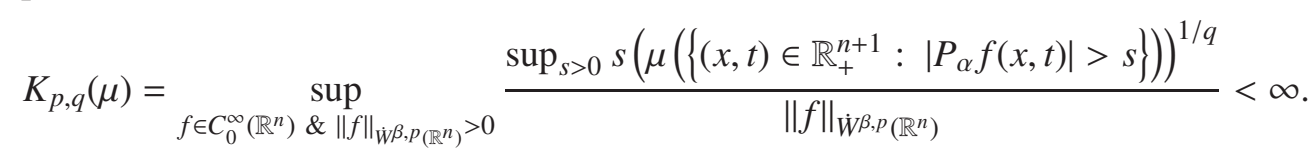


Then, for any $f \in C_{0}^{\infty}\left(\mathbb{R}^{n}\right)$ and any open set $O \subseteq$ Int $\left(\left\{x \in \mathbb{R}^{n}: f(x) \geq 1\right\}\right)$, (iv) of Lemma2.12 implies $T(O) \subset L_{\eta_{n, \alpha}}^{\alpha, \beta}(f)$, thus

$$
\mu(T(O)) \leq \mu\left(L_{\eta_{n, \alpha}}^{\alpha, \beta}(f)\right) \lesssim\left(K_{p, q}(\mu)\|f\|_{\dot{W}^{\beta, p}\left(\mathbb{R}^{n}\right)}\right)^{q} .
$$

Thus (v) holds.

For $(v) \Longrightarrow(i)$, denote

$$
Q_{p, q}(\mu):=\sup \left\{\frac{(\mu(T(O)))^{p / q}}{\operatorname{Cap}_{\mathbb{R}^{n}}^{\beta, p}(O)}: \text { bounded open } O \subseteq \mathbb{R}^{n}\right\}<\infty .
$$

Lemmas $2.12 \& 2.13$ imply

$$
\begin{aligned}
& \int_{0}^{\infty}\left(\mu\left(L_{s}^{\alpha, \beta}\right)(f) \cap T(B(0, k))\right)^{p / q} d s^{p} \\
& \quad \leq \int_{0}^{\infty}\left(\mu\left(T\left(R_{s}^{\alpha, \beta}(f) \cap B(0, k)\right)\right)^{p / q} d s^{p}\right. \\
& \quad \leq \int_{0}^{\infty}\left(\mu\left(T\left(\left\{x \in \mathbb{R}^{n}: \theta_{\alpha} \mathcal{M}(f)(x)>s\right\} \cap B(0, k)\right)\right)^{p / q} d s^{p}\right. \\
& \quad \leq Q_{p, q}(\mu) \int_{0}^{\infty}\left(C a p_{\mathbb{R}^{n}}^{\beta, p}\left(\left\{x \in \mathbb{R}^{n}: \theta_{\alpha} \mathcal{M}(f)(x)>s\right\}\right)\right) d s^{p} \\
& \leq Q_{p, q}(\mu)\|f\|_{\dot{W}^{\beta, p}\left(\mathbb{R}^{n}\right)}^{p}
\end{aligned}
$$

for any $f \in C_{0}^{\infty}\left(\mathbb{R}^{n}\right)$. Letting $k \longrightarrow \infty$ reaches (i).

Now, we will prove (iii) $\Longrightarrow$ (iv) $\Longrightarrow$ (i). If (iii) is true, then (4.1) implies $t^{p / q} \lesssim\left(K_{p, q}(\mu)\right)^{p} C a p_{\mathbb{R}^{n}}^{\beta, p}(O)$ whenever $t \in(0, \mu(T(O)))$. So, $t^{p / q} \lesssim\left(K_{p, q}(\mu)\right)^{p} c_{p}^{\beta}(\mu ; t)$. Thus, (iv) is true.

Assume (iv) is true. By Lemma 2.12 and Lemma2.13, for any $f \in C_{0}^{\infty}\left(\mathbb{R}^{n}\right)$,

$$
\begin{aligned}
& \int_{0}^{\infty}\left(\mu\left(L_{s}^{\alpha, \beta}\right)(f) \cap T(B(0, k))\right)^{p / q} d s^{p} \\
& \quad \leq \int_{0}^{\infty} \frac{\left(\mu\left(L_{s}^{\alpha, \beta}(f) \cap B(0, k)\right)\right)^{p / q}}{c_{p}^{\beta}\left(\mu ; \mu\left(L_{s}^{\alpha, \beta}(f) \cap B(0, k)\right)\right)} C a p_{\mathbb{R}^{n}}^{\beta, p}\left(R_{s}^{\alpha, \beta}(f) \cap B(0, k)\right) d s^{p} \\
& \quad \lesssim \sup _{t>0} \frac{t^{p / q}}{c_{p}^{\beta}(\mu ; t)} \int_{0}^{\infty} C a p_{\mathbb{R}^{n}}^{\beta, p}\left(\left\{x \in \mathbb{R}^{n}: \theta_{\alpha} \mathcal{M}(f)(x)>s\right\} \cap B(0, k)\right) d s^{p} \\
& \quad \lesssim \sup _{t>0} \frac{t^{p / q}}{c_{p}^{\beta}(\mu ; t)}\|f\|_{\dot{W}^{\beta, p}\left(\mathbb{R}^{n}\right)}^{p},
\end{aligned}
$$

which gives (i) via letting $k \longrightarrow \infty$.

Remark 4.2. Given $\beta \in(0, n), 1=p \leq \min \{n / \beta, q\}<\infty$ or $1<p<\min \{n / \beta, q\}$. Following the idea of Xiao in [29, Theorem 4.2] or that of Zhai in [31, Theorem 1.4], we can deduce from Lemma 2.15 that the condition $(v)$ in the equivalence of $(\mathrm{i}) \equiv(\mathrm{v})$ of Proposition 4.1 can be replaced by

$$
\sup _{x \in \mathbb{R}^{n}, r>0} \frac{(\mu(T(B(x, r))))^{1 / q}}{C a p_{\mathbb{R}^{n}}^{\beta, p}(B(x, r))}<\infty .
$$

Specially, when $1=p \leq \min \{n / \beta, q\}<\infty$ and $\beta \in(0, n)$, the equivalence $\operatorname{Cap}_{\dot{W}^{\beta, 1}} \approx H_{\infty}^{n-\beta}$ implies that $\operatorname{Cap}_{\mathbb{R}^{n}}^{\beta, p}(B(x, r))$ in (4.2) can be replaced by $H_{\infty}^{n-\beta}(B(x, r))$. Here $H_{\infty}^{d}(\cdot)$ denotes the $d$-dimensional Hausdorff capacity.

For the endpoint cases $p=1$ and $\beta \in(0,1)$, based on [30, Theorem 2], we can replace condition (v) of Proposition 4.1 by a condition in terms of the fractional perimeter of bounded open sets. 
Theorem 4.3. [30, Theorem 2] If $K$ is a compact subset of $\mathbb{R}^{n}$, then

$$
\operatorname{Cap}_{\mathbb{R}^{n}}^{\beta, 1}(K)=2 \inf _{O \in O^{\infty}(K)} \operatorname{Per}_{\beta}(O),
$$

where $O^{\infty}(K)$ is the class of all open sets with $C^{\infty}$ boundary that contain $K$.

Theorem 4.4. Let $\beta \in(0,1), 1=p \leq q<\infty$ and $\mu \in \mathcal{M}_{+}\left(\mathbb{R}_{+}^{n+1}\right)$. Then (1.5) is equivalent to

$$
\mu(T(O))^{1 / q} \lesssim \operatorname{Per}_{\beta}(O)
$$

for all bounded open sets $O \subseteq \mathbb{R}^{n}$.

Proof. It follows from Proposition 4.1 that (1.5) is equivalent to

$$
(\mu(T(O)))^{1 / q} \lesssim C a p_{\mathbb{R}^{n}}^{\beta, 1}(O)
$$

for all bounded open sets $O \subseteq \mathbb{R}^{n}$. Thus it suffices to show that (4.4) $\Longrightarrow(4.3) \Longrightarrow(1.5)$.

Firstly, we show that (4.4) $\Longrightarrow$ (4.3). Ponce-Spector in [22] proved that

$$
H_{\infty}^{n-\beta}(O) \lesssim \operatorname{Per}_{\beta}(O)
$$

holds for every bounded open sets $O \subseteq \mathbb{R}^{n}$. According to [2, 3], we have $\operatorname{Cap}_{\mathbb{R}^{n}}^{\beta, 1}(\cdot) \approx H_{\infty}^{n-\beta}(\cdot)$. Then 44.3) follows from

$$
(\mu(T(O)))^{1 / / q} \lesssim \operatorname{Cap}_{\mathbb{R}^{n}}^{\beta, 1}(O) \lesssim H_{\infty}^{n-\beta}(O) \lesssim \operatorname{Per}_{\beta}(O) .
$$

For 4.301 .5 , denote

$$
Q_{q}(\mu):=\sup \frac{(\mu(T(O)))^{1 / q}}{\operatorname{Per}_{\beta}(O)}<\infty,
$$

where the supermum is taken over all bounded open sets $O \subseteq \mathbb{R}^{n}$. For any $f \in C_{0}^{\infty}\left(\mathbb{R}^{n}\right)$ and any integer $k$, denote

$$
V=\left\{x \in \mathbb{R}^{n}: \theta_{\alpha} \mathcal{M}(f)(x)>s\right\} \cap B(0, k) .
$$

It follows from Theorem 4.3 that

$$
\operatorname{Cap}_{\mathbb{R}^{n}}^{\beta, 1}(\bar{V})=2 \inf _{O \in O^{\infty}(K)} \operatorname{Per}_{\beta}(\bar{V}),
$$

where $O^{\infty}(K)$ is the class of all open sets with $C^{\infty}$ boundary that contain $\bar{V}$. Thus, we have

$$
\begin{aligned}
\mu(T(V))^{1 / q} & \leq \mu(T(\bar{V}))^{1 / q} \\
& \leq \inf _{O \in O^{\infty}(\bar{V})} \mu(T(O))^{1 / q} \\
& \leq Q_{q}(\mu) \inf _{O \in O^{\infty}(\bar{V})} \operatorname{Per}_{\beta}(O) \\
& =\frac{Q_{q}(\mu)}{2} \operatorname{Cap}_{\mathbb{R}^{n}}^{\beta, 1}(\bar{V}) \\
& \lesssim \operatorname{Cap}_{\mathbb{R}^{n}}^{\beta, 1}\left(\left\{x \in \mathbb{R}^{n}: \theta_{\alpha} \mathcal{M}(f)(x) \geq s\right\}\right) .
\end{aligned}
$$

It follows from Lemma 2.12 and Lemma 2.13 that, for any $f \in C_{0}^{\infty}\left(\mathbb{R}^{n}\right)$,

$$
\begin{aligned}
\int_{0}^{\infty}\left(\mu\left(L_{s}^{\alpha, \beta}\right)(f) \cap T(B(0, k))\right)^{1 / q} d s & \leq \int_{0}^{\infty}\left(\mu\left(T\left(R_{s}^{\alpha, \beta}(f) \cap B(0, k)\right)\right)^{1 / q} d s\right. \\
& \leq \int_{0}^{\infty}\left(\mu\left(T\left(\left\{x \in \mathbb{R}^{n}: \theta_{\alpha} \mathcal{M}(f)(x)>s\right\} \cap B(0, k)\right)\right)^{1 / q} d s\right. \\
& \lesssim \int_{0}^{\infty} \operatorname{Cap} \mathbb{R}^{\beta, 1}\left(\left\{x \in \mathbb{R}^{n}: \theta_{\alpha} \mathcal{M}(f)(x) \geq s\right\}\right) d s \\
& \lesssim\|f\|_{\dot{W}^{\beta, 1}\left(\mathbb{R}^{n}\right)},
\end{aligned}
$$

which reaches 1.5 by letting $k \longrightarrow \infty$. 
Remark 4.5. It follows from Remark 4.2 and $\operatorname{Cap}_{\mathbb{R}^{n}}^{\beta, p}(B(x, r))=r^{n-\beta} \operatorname{Per}_{\beta}(B(0,1))$, see Xiao [30, Themrem 4], that $\operatorname{Per}_{\beta}(O)$ in (4.3) of Theorem 4.4 can be also replaced by $r^{n-\beta} \operatorname{Per}_{\beta}(B(0,1))$ when $\beta \in(0,1)$.

4.2. Embeddings of $\dot{W}^{\beta, p}\left(\mathbb{R}^{n}\right)$ to $L^{q}\left(\mathbb{R}_{+}^{n+1}, \mu\right)$ when $\max \{q, 1\}<p \leq n / \beta$.

Theorem 4.6. Let $\beta \in(0, n), 0<q<p, 1<p \leq n / \beta$ and $\mu \in \mathcal{M}_{+}\left(\mathbb{R}_{+}^{n+1}\right)$. Then (1.5) is equivalent to

$$
\int_{0}^{\infty}\left(\frac{t^{p / q}}{c_{p}^{\beta}(\mu ; t)}\right)^{q /(p-q)} \frac{d t}{t}<\infty .
$$

Proof. 4.5$) \Rightarrow$ (1.5). If

$$
I_{p, q}(\mu):=\int_{0}^{\infty}\left(\frac{t^{p / q}}{c_{p}^{\beta}(\mu ; t)}\right)^{q /(p-q)} \frac{d t}{t}<\infty,
$$

then for each $f \in C_{0}^{\infty}\left(\mathbb{R}^{n}\right)$, each $j=0, \pm 1, \pm 2, \ldots$, and each natural number $k$, Lemma2.12(iii) implies

$$
C a p_{\mathbb{R}^{n}}^{\beta, p}\left(R_{2^{j}}^{\alpha, \beta}(f) \cap(B(0, k))\right) \leq \operatorname{Cap}_{\mathbb{R}^{n}}^{\beta, p}\left(\left\{x \in \mathbb{R}^{n}: \theta_{\alpha} \mathcal{M} f(x)>2^{j}\right\} \cap B(0, k)\right) .
$$

Define

$$
\left\{\begin{array}{l}
\mu_{j, k}(f)=\mu\left(T\left(R_{2^{j}}^{\alpha, \beta}(f) \cap(B(0, k))\right)\right), \\
S_{p, q, k}(\mu ; f)=\sum_{j=-\infty}^{\infty} \frac{\left(\mu_{j, k}(f)-\mu_{j+1, k}(f)\right)^{p /(p-q)}}{\left(\operatorname{Cap}_{\mathbb{R}^{n}}^{\beta, p}\left(R_{2^{j}}^{\alpha, \beta}(f) \cap(B(0, k))\right)\right)^{q /(p-q)}} .
\end{array}\right.
$$

It follows from (ii) of Lemma 2.12 that

$$
\begin{aligned}
\left(S_{p, q, k}(\mu ; f)\right)^{(p-q) / p} & \lesssim\left(\sum_{j=-\infty}^{\infty} \frac{\mu_{j, k}^{q /(p-q)}(f)-\mu_{j+1, k}^{p /(p-q)}(f)}{\left(c_{p}^{\beta}\left(\mu ; \mu_{j, k}(f)\right)\right)^{q /(p-q)}}\right)^{(p-q) / p} \\
& \lesssim\left(\int_{0}^{\infty} \frac{1}{\left(c_{p}^{\beta}(\mu ; s)\right)^{q /(p-q)}} d s^{p /(p-q)}\right)^{(p-q) / p} \\
& \simeq\left(I_{p, q}(\mu)\right)^{(p-q) / p} .
\end{aligned}
$$

On the other hand, it follows from the Hölder inequality, (ii) of Lemma 2.13 and (ii)-(iii) of Lemma 2.12 that

$$
\begin{aligned}
& \int_{T(B(0, k))}\left|P_{\alpha} f(x, t)\right|^{q} d \mu(x, t) \\
& \quad=\int_{0}^{\infty} \mu\left(L_{s}^{\alpha, \beta}(f) \cap T(B(0, k))\right) d s^{q} \\
& \quad \lesssim \sum_{j=-\infty}^{\infty}\left(\mu_{j, k}(f)-\mu_{j+1, k}(f)\right) 2^{j q} \\
& \quad \lesssim\left(S_{p, q, k}(\mu ; f)\right)^{(p-q) / p}\left(\sum_{j=-\infty}^{\infty} 2^{j p} C_{a p}^{\beta, p}\left(R_{\mathbb{R}^{n}}^{\alpha, \beta}(f) \cap(B(0, k))\right)^{q / p}\right. \\
& \quad \lesssim\left(S_{p, q, k}(\mu ; f)\right)^{(p-q) / p}\left(\int_{0}^{\infty} C_{a p_{\mathbb{R}^{n}}^{\beta, p}}\left(\left\{x \in \mathbb{R}^{N}: \theta_{\alpha} \mathcal{M} f(x)>s\right\} \cap(B(0, k)) d s^{p}\right)^{q / p}\right. \\
& \quad \lesssim\left(S_{p, q, k}(\mu ; f)\right)^{(p-q) / p}\|f\|_{\dot{W}^{\beta, p}\left(\mathbb{R}^{n}\right)}^{q} .
\end{aligned}
$$


So, we get

$$
\left(\int_{T(B(0, k))}\left|P_{\alpha} f(x, t)\right|^{q} d \mu(x, t)\right)^{1 / q} \lesssim\left(I_{p, q}(\mu)\right)^{(p-q) / p q}\|f\|_{\dot{W}^{\beta, p}\left(\mathbb{R}^{n}\right)} .
$$

Letting $k \longrightarrow \infty$ derives $(1.5)$.

(1.5) $\Rightarrow(4.5$ ). If (1.5) holds, then

$$
C_{p, q}(\mu):=\sup _{f \in C_{0}^{\infty}\left(\mathbb{R}^{n}\right),\|f\|_{\left.\dot{W}^{\beta, p}, \mathbb{R}^{n}\right)}>0} \frac{1}{\|f\|_{\dot{W}^{\beta, p}\left(\mathbb{R}^{n}\right)}}\left(\int_{\mathbb{R}_{+}^{n+1}}\left|P_{\alpha} f(x, t)\right|^{q} d \mu(x, t)\right)^{1 / q}<\infty .
$$

Then for each $f \in C_{0}^{\infty}\left(\mathbb{R}^{n}\right)$ with $\|f\|_{\dot{W}^{\beta, p}\left(\mathbb{R}^{n}\right)}>0$, there holds

$$
\left(\int_{\mathbb{R}_{+}^{n+1}}\left|P_{\alpha} f(x, t)\right|^{q} d \mu(x, t)\right)^{1 / q} \leq C_{p, q}(\mu)\|f\|_{\dot{W}^{\beta, p}\left(\mathbb{R}^{n}\right)},
$$

which implies by Markov's inequality

$$
\sup _{s>0} s\left(\mu\left(L_{s}^{\alpha, \beta}(f)\right)\right)^{1 / q} \lesssim C_{p, q}(\mu)\|f\|_{\dot{W}^{\beta, p}\left(\mathbb{R}^{n}\right)} .
$$

This, together with (iv) of Lemma 2.12, implies that for fixed $f \in C_{0}^{\infty}\left(\mathbb{R}^{n}\right)$,

$$
\mu(T(O)) \lesssim C_{p, q}^{q}(\mu)\|f\|_{\dot{W}^{\beta, p}\left(\mathbb{R}^{n}\right)}^{q}
$$

for any bounded open set $O \subseteq \operatorname{Int}\left(\left\{x \in \mathbb{R}^{n}: f(x) \geq 1\right\}\right)$. The definition of $c_{p}^{\beta}(\mu ; t)$ implies that $c_{p}^{\beta}(\mu ; t)>0$, and for $t \in(0, \infty)$ and every $j$ there exists a bounded open set $O_{j} \subseteq \mathbb{R}^{n}$ such that $\operatorname{Cap}_{\mathbb{R}^{n}}^{\beta, p}\left(O_{j}\right) \leq 2 c_{p}^{\beta}\left(\mu ; 2^{j}\right)$ and $\mu\left(T\left(O_{j}\right)\right)>2^{j}$.

When $p \in(1, n / \beta)$, according to [20, Theorem 2, P. 347], $f \in \dot{W}^{\beta, p}\left(\mathbb{R}^{n}\right)$ when $p \in(1, n / \beta)$ if and only if $f=c I_{\beta} * g(x)$ for some $f \in L^{p}\left(\mathbb{R}^{n}\right)$. Here $I_{\beta} * g(x)=\int_{\mathbb{R}^{n}} \frac{f(y)}{|x-y|^{n-\beta}} d y$. Thus, $\|f\|_{\dot{W}^{\beta, p}\left(\mathbb{R}^{n}\right)}=\left\|(-\triangle)^{\beta / 2} f\right\|_{L^{p}\left(\mathbb{R}^{n}\right)} \approx$ $\left\|(-\Delta)^{\beta / 2}\left(I_{\beta} * g\right)\right\|_{L^{p}\left(\mathbb{R}^{n}\right)} \approx\|g\|_{L^{p}\left(\mathbb{R}^{n}\right)}$. So, we have

$$
\operatorname{Cap}_{\mathbb{R}^{n}}^{\beta, p}(S) \approx \inf \left\{\|g\|_{L^{p}\left(\mathbb{R}^{n}\right)}^{p}: g \in C_{0}^{\infty}\left(\mathbb{R}^{n}\right), g \geq 0, S \subseteq \operatorname{Int}\left(\left\{x \in \mathbb{R}^{n}: I_{\beta} * g(x) \geq 1\right\}\right)\right\} .
$$

Then, there exists $g_{j} \in C_{0}^{\infty}\left(\mathbb{R}^{n}\right)$ such that $I_{\beta} * g_{j}(x) \geq 1$ on $O_{j}$, and

$$
\left\|g_{j}\right\|_{L^{p}\left(\mathbb{R}^{n}\right)}^{p} \leq 2 \operatorname{Cap}_{\mathbb{R}^{n}}^{\beta, p}\left(O_{j}\right) \leq 4 c_{p}^{\beta}\left(\mu ; 2^{j}\right) .
$$

For the integers $i, k$ with $i<k$, define

$$
g_{i, k}=\sup _{i \leq j \leq k}\left(\frac{2^{j}}{c_{p}^{\beta}\left(\mu ; 2^{j}\right)}\right)^{\frac{1}{p-q}} g_{j}
$$

Then $g_{i, k} \in L^{p}\left(\mathbb{R}^{n}\right) \cap L_{l o c}^{1}\left(\mathbb{R}^{n}\right)$ with

$$
\left\|g_{i, k}\right\|_{L^{p}\left(\mathbb{R}^{n}\right)}^{p} \lesssim \sum_{j=i}^{k}\left(\frac{2^{j}}{c_{p}^{\beta}\left(\mu ; 2^{j}\right)}\right)^{\frac{p}{p-q}} c_{p}^{\beta}\left(\mu ; 2^{j}\right) .
$$

On the other hand, $I_{\beta} * g_{i, k} \in \dot{W}^{\beta, p}\left(\mathbb{R}^{n}\right)$ since $I_{\beta}: \dot{F}_{p, 2}^{0}\left(\mathbb{R}^{n}\right)=L^{p}\left(\mathbb{R}^{n}\right) \rightarrow \dot{F}_{p, 2}^{\beta}=\dot{W}^{\beta, p}\left(\mathbb{R}^{n}\right)$, see [27, Theorem 1, page 242]

Since for $i \leq j \leq k$,

$$
x \in O_{j} \Rightarrow I_{\beta} * g_{i, k}(x) \geq\left(\frac{2^{j}}{c_{p}^{\beta}\left(\mu ; 2^{j}\right)}\right)^{\frac{1}{p-q}}
$$


(iv) of Lemma2.12 implies that there exists a constant $\eta_{n, \alpha}$ such that

$$
(x, t) \in T\left(O_{j}\right) \Rightarrow\left|u_{i, k}(x, t)\right| \geq\left(\frac{2^{j}}{c_{p}^{\beta}\left(\mu ; 2^{j}\right)}\right)^{\frac{1}{p-q}} \eta_{n, \alpha} .
$$

Here $u_{i, k}$ denotes the Caffarelli-Silvestre extension of $I_{\beta} * g_{i, k}$. Thus, with $s=\left(\frac{2^{j}}{c_{p}^{\beta}\left(\mu 2^{j}\right)}\right)^{\frac{1}{p-q}} \frac{\eta_{n, \alpha}}{2}$,

$$
2^{j} \leq \mu\left(T\left(O_{j}\right)\right) \leq \mu\left(L_{s}^{\alpha, \beta}\left(I_{\beta} * g_{i, k}\right)\right) .
$$

Similar to that in the the proof of (i) in Theorem 3.4, we can get

$$
\begin{aligned}
\left(C_{p, q}(\mu)\left\|g_{i, k}\right\|_{L^{p}\left(\mathbb{R}^{n}\right)}\right)^{q} & \gtrsim \int_{\mathbb{R}_{+}^{n+1}}\left|u_{i, k}(x, t)\right| d \mu \\
& \approx \int_{0}^{\infty}\left(\inf \left\{s: \mu\left(L_{s}^{\alpha, \beta}\right)\left(I_{\beta} * g_{i, k}\right) \leq s\right\}\right)^{q} d s \\
& \gtrsim \sum_{j=i}^{k}\left(\inf \left\{s: \mu\left(L_{s}^{\alpha, \beta}\right)\left(I_{\beta} * g_{i, k}\right) \leq 2^{j}\right\}\right)^{q} 2^{j} \\
& \gtrsim \sum_{j=i}^{k}\left(\frac{2^{j}}{c_{p}^{\beta}\left(\mu ; 2^{j}\right)}\right)^{\frac{q}{p-q}} 2^{j} \\
& \gtrsim\left(\sum_{j=i}^{k} \frac{2^{j p /(p-q)}}{c_{p}^{\beta}\left(\mu ; 2^{j}\right)^{q /(p-q)}}\right)^{\frac{p-q}{p}}\left\|g_{i, k}\right\|_{L^{p}\left(\mathbb{R}^{n}\right)}^{q},
\end{aligned}
$$

where in the last step, we used (4.6). Thus,

$$
\sum_{j=i}^{k} \frac{2^{j p /(p-q)}}{c_{p}^{\beta}\left(\mu ; 2^{j}\right)^{q /(p-q)}} \lesssim\left(C_{p, q}(\mu)\right)^{p q /(p-q)} .
$$

When $p=n / \beta$, the definition of $\operatorname{Cap}_{\mathbb{R}^{n}}^{\beta, p}(\cdot)$ implies that there exists a positive $f_{j} \in C_{0}^{\infty}\left(\mathbb{R}^{n}\right)$ such that $f_{j} \geq 1$ on $O_{j}$ and

$$
\left\|f_{j}\right\|_{\dot{W}^{\beta, p}\left(\mathbb{R}^{n}\right)}^{p} \leq 2 \operatorname{Cap}_{\mathbb{R}^{n}}^{\beta, p}\left(O_{j}\right) \leq 4 c_{p}^{\beta}\left(\mu ; 2^{j}\right)
$$

Define $g_{j}(x, h):=|h|^{-2 \beta} \triangle_{h}^{k} f_{j}(x)$. Then $g_{j} \in L^{p}\left(\mathbb{R}^{2 n}\right) \cap L_{l o c}^{1}\left(\mathbb{R}^{2 n}\right)$. On the other hand, [1, Theorem 5.1] implies $f_{j}(x)=C I_{2 \beta}^{(2 n)} * g_{j}(x, 0)$, for a.e. $x \in \mathbb{R}^{n}$.

For the integers $i, k$ with $i<k$, define

$$
g_{i, k}(x, h)=\sup _{i \leq j \leq k}\left(\frac{2^{j}}{c_{p}^{\beta}\left(\mu ; 2^{j}\right)}\right)^{\frac{1}{p-q}} g_{j}(x, h) .
$$

Then $g_{i, k} \in L^{p}\left(\mathbb{R}^{2 n}\right) \cap L_{l o c}^{1}\left(\mathbb{R}^{2 n}\right)$, and Lemma 2.14 implies

$$
\begin{aligned}
\left\|\mathcal{R} I_{2 \beta}^{(2 n)} * g_{i, k}\right\|_{\dot{W}^{\beta, p}\left(\mathbb{R}^{n}\right)}^{p} & \lesssim \sum_{j=i}^{k}\left(\frac{2^{j}}{c_{p}^{\beta}\left(\mu ; 2^{j}\right)}\right)^{\frac{p}{p-q}}\left\|I_{2 \beta}^{(2 n)} * g_{i, k}\right\|_{\dot{L}^{p}\left(\mathbb{R}^{2 n}\right)}^{p} \\
& \lesssim \sum_{j=i}^{k}\left(\frac{2^{j}}{c_{p}^{\beta}\left(\mu ; 2^{j}\right)}\right)^{\frac{p}{p-q}}\left\|f_{j}\right\|_{\dot{W}^{\beta, p}\left(\mathbb{R}^{n}\right)}^{p} \\
& \lesssim \sum_{j=i}^{k}\left(\frac{2^{j}}{c_{p}^{\beta}\left(\mu ; 2^{j}\right)}\right)^{\frac{p}{p-q}} c_{p}^{\beta}\left(\mu ; 2^{j}\right) .
\end{aligned}
$$


Let $u_{i, k}(x, t)$ be the Caffarelli-Silvestre extension of $\mathcal{R}\left(I_{2 \beta}^{(2 n)} g_{i, k}\right)(x)$. Then, similar to the previous case, for $i \leq j \leq k$,

$$
x \in O_{j} \Rightarrow \mathcal{R}\left(I_{2 \beta}^{(2 n)} g_{i, k}\right)(x) \geq\left(\frac{2^{j}}{c_{p}^{\beta}\left(\mu ; 2^{j}\right)}\right)^{\frac{1}{p-q}} .
$$

(iv) of Lemma2.12 implies that there exists a constant $\eta_{n, \alpha}$ such that

$$
(x, t) \in T\left(O_{j}\right) \Rightarrow\left|u_{i, k}(x, t)\right| \geq\left(\frac{2^{j}}{c_{p}^{\beta}\left(\mu ; 2^{j}\right)}\right)^{\frac{1}{p-q}} \eta_{n, \alpha}
$$

and with $s=\left(\frac{2^{j}}{c_{p}^{\beta}\left(\mu ; 2^{j}\right)}\right)^{\frac{1}{p-q}} \frac{\eta_{n, \alpha}}{2}$

$$
2^{j} \leq \mu\left(T\left(O_{j}\right)\right) \leq \mu\left(L_{s}^{\alpha, \beta}\left(\mathcal{R}\left(I_{2 \beta}^{(2 n)} g_{i, k}\right)\right)\right)
$$

We can get

$$
\begin{aligned}
\left(C_{p, q}(\mu)\left\|\mathcal{R}\left(I_{2 \beta}^{(2 n)} g_{i, k}\right)\right\|_{\dot{W}^{\beta, p}\left(\mathbb{R}^{n}\right)}\right)^{q} & \gtrsim \int_{\mathbb{R}_{+}^{n+1}}\left|u_{i, k}(x, t)\right| d \mu \\
& \approx \int_{0}^{\infty}\left(\inf \left\{s: \mu\left(L_{s}^{\alpha, \beta}\left(\mathcal{R}\left(I_{2 \beta}^{(2 n)} g_{i, k}\right)\right)\right) \leq s\right\}\right)^{q} d s \\
& \gtrsim \sum_{j=i}^{k}\left(\inf \left\{s: \mu\left(L_{s}^{\alpha, \beta}\left(\mathcal{R}\left(I_{2 \beta}^{(2 n)} g_{i, k}\right)\right)\right) \leq 2^{j}\right\}\right)^{q} 2^{j} \\
& \gtrsim \sum_{j=i}^{k}\left(\frac{2^{j}}{c_{p}^{\beta}\left(\mu ; 2^{j}\right)}\right)^{\frac{q}{p-q}} 2^{j} \\
& \gtrsim\left(\sum_{j=i}^{k} \frac{2^{j p /(p-q)}}{c_{p}^{\beta}\left(\mu ; 2^{j}\right)^{q /(p-q)}}\right)^{\frac{p-q}{p}} \| \mathcal{R}\left(I_{2 \beta}^{(2 n)} g_{i, k} \|_{\dot{W}^{\beta, p}\left(\mathbb{R}^{n}\right)}^{q}\right.
\end{aligned}
$$

Thus, we have

$$
\sum_{j=i}^{k} \frac{2^{\frac{j p}{(p-q)}}}{c_{p}^{\beta}\left(\mu ; 2^{j}\right)^{\frac{q}{p-q}}} \lesssim\left(C_{p, q}(\mu)\right)^{\frac{p q}{p-q}}
$$

Letting $i, k \longrightarrow \infty$, we reach

$$
\int_{0}^{\infty}\left(\frac{t^{p / q}}{c_{p}^{\beta}(\mu ; t)}\right)^{\frac{q}{p-q}} \lesssim \sum_{-\infty}^{\infty} \frac{2^{\frac{j p}{p-q}}}{\left(c_{p}^{\beta}\left(\mu ; 2^{j}\right)\right)^{\frac{q}{(p-q)}}} \lesssim\left(C_{p, q}(\mu)\right)^{\frac{p q}{p-q}}
$$

which implies 4.5.

When $q<p=1$, we can establish the following necessary conditions for the embedding (1.5).

Proposition 4.7. Let $\beta \in(0, n), 0<q<p=1$ and $\mu \in \mathcal{M}_{+}\left(\mathbb{R}_{+}^{n+1}\right)$. Then $(1.5) \Rightarrow(i) \Rightarrow$ (ii) $\Rightarrow$ (iii):

(i) $\left\|P_{\alpha} f(\cdot, \cdot)\right\|_{L^{q, \infty}\left(\mathbb{R}_{+}^{n+1}, \mu\right)} \lesssim\|f\|_{W^{\beta, 1}\left(\mathbb{R}^{n}\right)} \quad \forall f \in C_{0}^{\infty}\left(\mathbb{R}^{n}\right)$;

(ii) $\sup \left\{\frac{(\mu(T(O)))^{1 / q}}{\operatorname{Cap}_{\mathbb{R}^{n}}(O)}\right.$ : bounded open set $\left.O \subseteq \mathbb{R}^{n}\right\}<\infty$;

(iii) $\left\|P_{\alpha} f(\cdot, \cdot)\right\|_{L^{q, 1}\left(\mathbb{R}_{+}^{n+1}, \mu\right)} \lesssim\|f\|_{\dot{W}^{\beta, 1}\left(\mathbb{R}^{n}\right)} \quad \forall f \in C_{0}^{\infty}\left(\mathbb{R}^{n}\right)$. 
Proof. The proofs of $(1.5) \Longrightarrow$ (i) $\Longrightarrow$ (ii) are similar to those of (ii) $\Longrightarrow$ (iii) $\Longrightarrow$ (v) in the proof of Proposition 4.1, For the implication (ii) $\Longrightarrow$ (iii), firstly, there holds

$$
\begin{aligned}
\mu\left(L_{s}^{\alpha, \beta}(f)\right) & \leq \mu\left(T\left(R_{s}^{\alpha, \beta}(f)\right)\right) \\
& \leq \mu\left(T\left(\left\{x \in \mathbb{R}^{n}: \theta_{\alpha} \mathcal{M} f(x)>s\right\}\right)\right) \\
& \lesssim\left(\operatorname{Cap}_{\mathbb{R}^{n}}^{\beta, 1}\left(\left\{x \in \mathbb{R}^{n}: \theta_{\alpha} \mathcal{M} f(x)>s\right\}\right)\right)^{q},
\end{aligned}
$$

due to Lemma 2.12. Then, applying (ii) of Lemma 2.13 we can show (ii) $\Longrightarrow$ (iii) in a similar way as the proof of $(\mathrm{v}) \Longrightarrow(\mathrm{i})$ in Proposition 4.1

\section{ACKNOWLEDGEMENT}

The authors are very grateful to the anonymous reviewers, especially one of whom gave numerous useful suggestions that lead to a substantial improvement of this manuscript.

\section{REFERENCES}

[1] D. R. Adams, Lectures on $L_{p}$ potential Theory, Univ. of Umea, 1981.

[2] D. R. Adams, The classification problem for the capacities associated with the Besov and Triebel-Lizorkin spaces,in: Banach Center Publ., vol. 22, 1989, pp. 9-24.

[3] D. R. Adams, Choquet integrals in potential theory, Publ. Mat. 42 (1998), 3-66.

[4] D. R. Adams, L. I. Hedberg, Function Spaces and Potential Theory. Berlin: Springer, 1996.

[5] D. R. Adams and J. Xiao, Strong type estimates for homogeneous Besov capacities, Math. Ann. 325 (2003), 695-709.

[6] L. Ambrosio, G. D. Philippis and L. Martinazzi, Gamma-convergence of nonlocal perimeter functionals, Manuscripta Math. 134 (2011), 377-403.

[7] C. Bennett, RC. Sharpley, Interpolation of operators. Academic press, 1988.

[8] C. Brändle, E. Colorado, A. de Pablo and U. Sánchez, A concave-convex elliptic problem involving the fractional Laplacian, Proc. R. Soc. Edinb. A 143 (2013), 39-71.

[9] H. Q. Bui, T. Candy, A characterization of the Besov-Lipschitz and Triebel-Lizorkin spaces using Poisson like kernels. (English summary) Functional analysis, harmonic analysis, and image processing: a collection of papers in honor of Björn Jawerth, 109-141, Contemp. Math., 693, Amer. Math. Soc., Providence, RI, 2017.

[10] L. Caffarelli, J. Roquejoffre and O. Savin, Non-local minimal surfaces, Comm. Pure Appl. Math. 63 (2010), $1111-1144$.

[11] L. Caffarelli and L. Silvestre, An extension problem related to the fractional Laplacian, Comm. Partial Diff. Equ. 32 (2007), 1245-1260.

[12] C. Cascante, J. Ortega and I. Verbitsky, Trace inequality of Sobolev type in the upper triangle cases, Proc. London Math. Soc. 80 (2000), 391-414.

[13] D. Chang and J. Xiao, $L^{q}$-embeddings of $L$-spaces by fractional diffusion equations, Discrete Contin. Dyn. Syst. 35 (2015), 1905-1920.

[14] P. DeNápoli, J. Haddad, C. Jiménez and M. Montenegro, The sharp affine $L^{2}$ Sobolev trace inequality and variants, Math. Ann. 370 (2018), 287-308.

[15] R. Fefferman, Strong differentiation with respect to measures, Amer. J. Math. 103 (1981), 33-40.

[16] N. Fusco, V. Millot and M. Morini, A quantitative isoperimetric inequality for fractional perimeters, J. Funct. Anal. 26 (2011), 697-715.

[17] R. Hurri-Syrjänen and A. Vähäkangas, Characterizations to the fractional Sobolev inequality In Complex analysis and dynamical systems VII, volume 699 of Contemp. Math. pages 145-154. Amer. Math. Soc., Providence, RI, 2017.

[18] R. Jiang, J. Xiao, D. Yang and Z. Zhai, Regularity and capacity for the fractional dissipative operator, J. Differential Equ. 259 (2015), 3495-3519.

[19] E. Lenzmann, A. Schikorra, Sharp commutator estimates via harmonic extensions. Nonlinear Anal. 193 (2020), 111375.

[20] V.G. Maz’ya, Sobolev Spaces, Springer, Berlin, New York, 1985.

[21] V. Nguyen, Some trace Hardy type inequalities and trace Hardy-Sobolev-Maz'ya type inequalities, J. Funct. Anal. 270 (2016), 4117-4151.

[22] A. Ponce and D. Spector, A boxing inequality for the fractional perimeter, Ann. Sc. Norm. Super. Pisa Cl. Sci (2020): 107-141.

[23] S. Shi and J. Xiao, Fractional capacities relative to bounded open Lipschitz sets, Potential Anal. 45 (2016), 261-298.

[24] S. Shi and J. Xiao, A tracing of the fractional temperature field, Sci. China Math. 60 (2017), 2303-2320. 
[25] E. M. Stein, Harmonic Analysis: Real-variable Methods, Orthogonality, and Oscillatory Integrals, Princeton University Press, Princeton, New Jersey, 1993.

[26] P. Stinga and J. Torrea, Extension problem and Harnack's inequality for some fractional operators, Commun. Partial Differ. Equ. 35 (2010), 2092-2122.

[27] H. Triebel, Theory of Function Spaces, Monogr. Math., vol. 78, Birkhäuser, Basel, 1983.

[28] J. Xiao, Carleson embeddings for Sobolev spaces via heat equation, J. Differential Equations 224 (2006), 277-295.

[29] J. Xiao, Homogeneous endpoint Besov space embeddings by Hausdorff capacity and heat equation, Adv. Math. 207 (2006), 828-846.

[30] J. Xiao, Optimal geometric estimates for fractional Sobolev capacities, C. R. Acad. Sci. Ser. I. 354 (2016), $149-153$.

[31] Z. Zhai, Carleson measure problems for parabolic Bergman spaces and homogeneous Sobolev spaces, Nonlinear Anal, 73 (2010), 2611-2630. 